Warsaw University of Technology Faculty of Electronics and Information Technology

Electrical and Computer Engineering

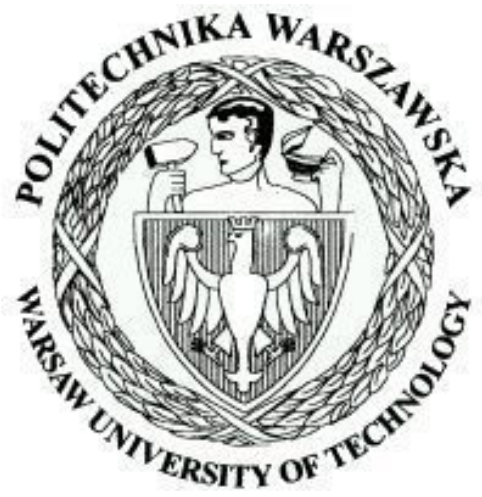

MASTER OF SCIENCE THESIS

Adrian Fiergolski

\title{
The Firmware of the Front-End Driver for the Data Acquisition System of the TOTEM Experiment
}

Supervisor:

dr Mariusz Rawski

Evaluation 


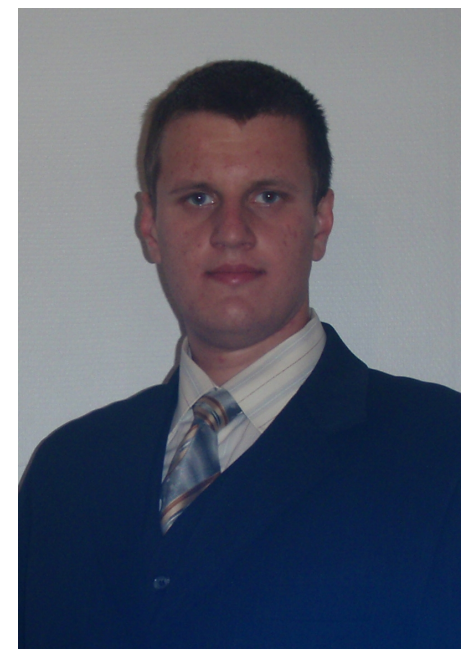

Field of Studies: Electrical

and Computer Engineering

Date of Birth:

15 October 1986

Starting Date of Studies: 1 October 2005

\section{Curriculum Vitae}

I was born on 15 October 1986 in Grudziądz, Poland. In 2004 I was a member of the team, which won the Provincial "Banach" Mathematical Competition. I graduated from the high school in Brodnica in 2005.

Since 2005 I am a student of the Faculty of Electronics and Information Technology of Warsaw University of Technology. I am a member of the DEMAIN group - science group of students who are interested in the FPGA technology. It gave me an opportunity to complete few interesting projects: porting uClinux system for an FPGA device and designing ALSA drivers for WM8731 codec; implementation of TCP/IP stack in FPGA using Lightweight IP; hardware implementation of AES algorithm or hardware implementation of DWT. In years 2006-2010 I was granted the scholarship of the Faculty for the best students.

I am interested in electronics (Programmable Logic - FPGA, Microcontrollers, Digital Signal Processing) and singing (I was a member of few choirs).

Signature of the Student

\section{Master of Science Examination}

Examination was held on:

With the result:

Final Result of the Studies:

Suggestions and Remarks of the B.Sc. Examination Committee: 


\begin{abstract}
The TOTEM experiment at the LHC at CERN will measure the total proton-proton cross section with precision of $1 \%$, elastic proton scattering and diffractive dissociation at the center of the mass energy of $14 \mathrm{TeV}$. This document is report on the TOTFed motherboard, which is the main part of the on-line Data Acquisition of the TOTEM. The designed firmware is supposed to collect data from the particle detectors, build a consistent event frame and send it to the counting room. The data processing is divided between 7 FPGAs. To accomplish the objective, specialized interfaces such as VME64x, S-Link 64, Gigabit Optical Link (GOL) are used. Since a significant part of the applied electronics is specific only for the LHC machine, the FPGA firmware implementation required a deep understanding of the Data Acquisition System and then intensive studies and debugging of the developed solutions.
\end{abstract}

Key words: TOTEM experiment, TOTFed firmware, DAQ

\title{
Streszczenie
}

Tytul: Firmware końcowego kontrolera systemu akwizycji danych $w$ eksperymencie TOTEM.

Eksperyment TOTEM dziatajacy przy LHC $w$ CERN'ie będzie mierzył catkowity przekrój czynny $w$ zderzeniach proton-proton $z$ dokładnościa $1 \%$, elastyczne rozpraszanie protonów i dysocjacje dyfrakcyjna przy energii $14 \mathrm{TeV} w$ centrum masy. Niniejszy dokument traktuje o TOTFed'dzie, głównym elemencie $w$ Systemie Akwizycji Danych TOTEM'u pracujacym $w$ czasie rzeczywistym. Zaprojektowany firmware ma za zadanie zbierać dane $z$ rejonu detektora, zbudować poprawna ramkę zdarzenia i wysłać ja do rejonu gdzie jest ona archiwizowana i później wykorzystywana $w$ celu rekonstrukcji zderzenia $w$ detektorze. Zadanie to jest dzielone pomiędzy 7 układów FPGA. Żeby sprostać postawionym wymaganiom, TOTFed wykorzystuje specjalizowane interfejsy takie jak VME64x, S-Link 64, czy Gigabit Optical Link (GOL). Duża cześć elektroniki jest specyficzna dla maszyny LHC, stad wysiłek wtożony $w$ implementacje wymagat wnikliwej analizy systemu i jego dokładnego testowania, aby uzyskać wiarygodne rozwiazanie.

Słowa kluczowe: eksperyment TOTEM, firmware TOTFed'a, DAQ 


\section{Contents}

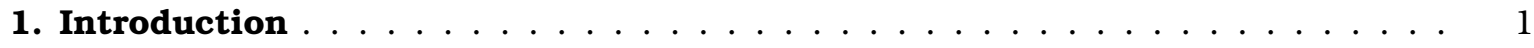

1.1. The Large Hadron Collider . . . . . . . . . . . . . . . . . . . . . . 1

1.2. Scope ................................ 2

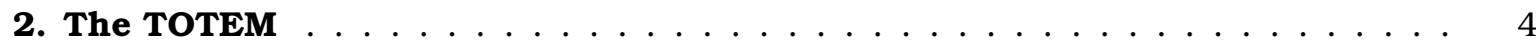

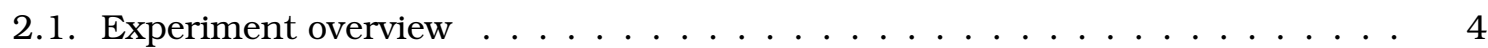

2.2. General overview of the TOTEM readout system . . . . . . . . . . . 5

2.3. TOTEM Local DAQ system $\ldots \ldots \ldots$. . . . . . . . . . . . . . . . . . . 6

2.3.1. Architecture of the readout chain . . . . . . . . . . . 6

2.3.2. The Control and Clock System . . . . . . . . . . . . . . 7

2.3.3. Timing, Trigger and Control System . . . . . . . . . . . . . . 8

2.3.4. Trigger Throttling System $\ldots \ldots \ldots$. . . . . . . . . . . . 9

2.4. The CMS Common Data format . . . . . . . . . . . . . . . . . . 10

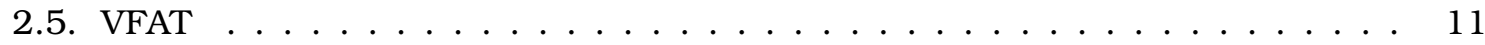

2.5.1. General description . . . . . . . . . . . . . . . 12

2.5.2. The VFAT data format . . . . . . . . . . . . . . . . . . 13

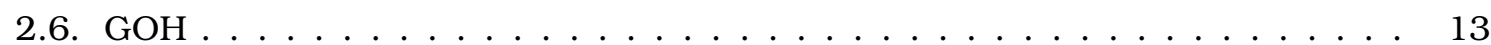

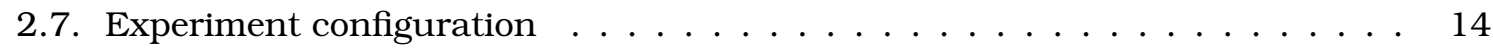

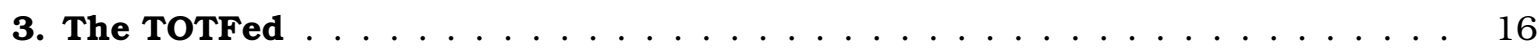

3.1. General Overview . . . . . . . . . . . . . . . . 16

3.2. TTC signals distribution on the TOTFed . . . . . . . . . . . . . . . . 18

3.3. Memory Space . . . . . . . . . . . . . . . . . . . . . . . . . 18

3.4. Reset system of the TOTFed . . . . . . . . . . . . . . . . . . 19

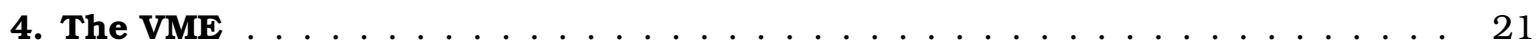

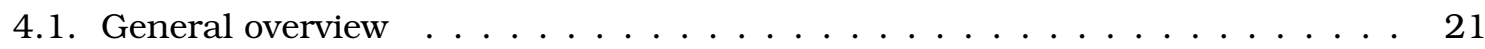

4.2. Implementation . . . . . . . . . . . . . . . . . . 23

4.2.1. The TTCrx Controller by I2C bus . . . . . . . . . . . . . . . 23

4.2.2. VME Reset Subsystem . . . . . . . . . . . . . . . . . . . . . 30

4.2.3. VME Controller . . . . . . . . . . . . . . . . . . 33

5. The Main . . . . . . . . . . . . . . . . . . . . . . . . . . . . . . . . 44

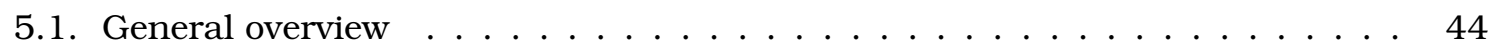

5.2. Implementation . . . . . . . . . . . . . . . . . . . 45

5.2.1. The Local bus slave controller . . . . . . . . . . . . . . . . . . 45

5.2.2. The OptoRx receiver $\ldots \ldots \ldots \ldots$. . . . . . . . . 53

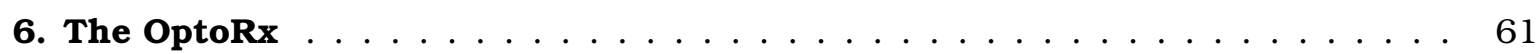

6.1. General Overview . . . . . . . . . . . . . . . . . 61

6.2. Implementation . . . . . . . . . . . . . . . . . . . . . 63

6.2.1. Data Emulator . . . . . . . . . . . . . . . . . . 63

6.2.2. Opto-local bus . . . . . . . . . . . . . . . . . . . . 69

6.2.3. TTCrx Receiver. . . . . . . . . . . . . . . . . . . 71

6.2.4. Channel data processor . . . . . . . . . . . . . . . 73

6.2.5. Data Processing . . . . . . . . . . . . . . . . 75

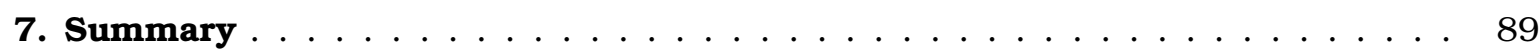

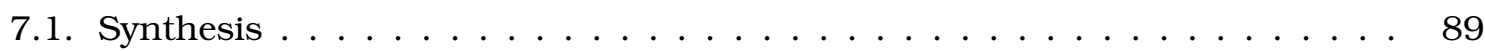


7.2. Real life tests . . . . . . . . . . . . . . . . . . . . . . . . 91

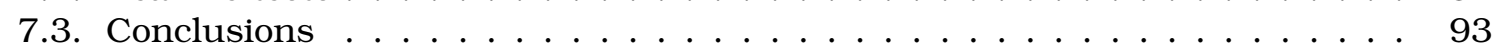

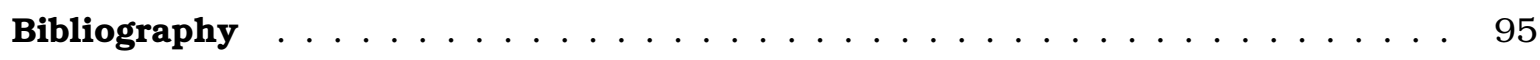




\section{Acknowledgements}

I would like to thank my CERN supervisors: Dr. Francesco Cafagna and Dr. Emilio Radicioni for all their knowledge, care, help and time their spent giving me explanations and plenty of important advices.

I am indebted to Dr. Mariusz Rawski - my supervisor from Warsaw University of Technology for his advices, support, supervision and for this thesis reading.

I am grateful to Michele Quinto with whom I have been developing the TOTFed firmware. Thank you for discussions, explanations and successful cooperation.

Also, I am very thankful to Dr. Huber Niewiadomski for his support, supervision, especially during first months of my stay at CERN and as well for reading the thesis.

I would like to express my deep gratitude to my family, my father Leszek, my sister Marta, my grandparent Zdzisława, my aunts: Barbara and Mariola and uncles: Roman and Marek. Without your support, this work would have not been possible.

Finally, I would like to greatly acknowledge CERN, the TOTEM Collaboration and the Warsaw University of Technology for support of funding this thesis.

Geneva, June 2010

Adrian Fiergolski

To my mother Aleksandra Although you can not read it, I hope, that you are proud of me. 


\section{Introduction}

\subsection{The Large Hadron Collider}

Our current understanding of the Universe is incomplete. The motivation to construct the Large Hadron Collider (LHC) at the European Center for Particle Physics (CERN) comes from the fundamental questions in Particle Physics. The complete understanding of the Standard Model and the search of the phenomena beyond it requires a high energy and a high luminosity collider.

The LHC is a circular accelerator with $26.7 \mathrm{~km}$ circumference located in the existing tunnel of the LEP (Large Electron Positron collider) at $50 \div 100$ m under ground level. The LHC reuses the existing accelerators as injectors. Prior to being injected into the main accelerator, the particles are prepared through a series of machines that successively increase the particle energy levels. The first system is the linear accelerator (Linac2) generating $50 \mathrm{MeV}$ protons which feeds the Proton Synchrotron Booster (PSB). Protons are then injected at $1.4 \mathrm{GeV}$ into the Proton Synchrotron (PS). Finally, the Super Proton Synchrotron (SPS) is used to increase the energy of protons from $26 \mathrm{GeV}$ up to $450 \mathrm{GeV}$. Such protons are injected to the LHC.

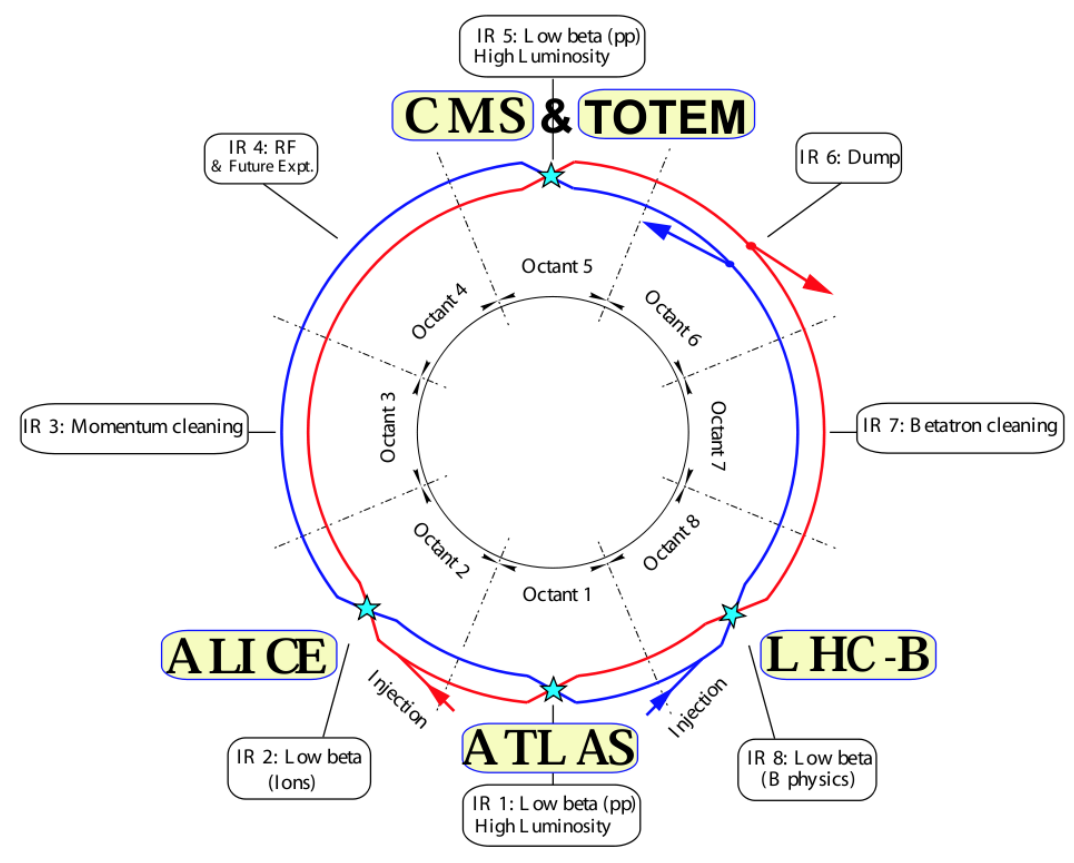

Figure 1.1. Schematic layout of the LHC. The four interaction points in which the beams circulating in opposite directions cross are indicated by stars. 
Two counter-rotating proton beams circulates in separate beam pipes installed in the common magnets. The protons will be accelerated up to an energy of $7 \mathrm{TeV}$. After having reached this energy these two beams, moving in opposite directions, will cross at four interaction points (IP) along the beam line. These are represented by stars in Figure 1.1 .

Six experiments will perform the high energy particle physics research in the LHC: A Large Ion Collider Experiment (ALICE), ATLAS, the Compact Muon Solenoid (CMS), the Large Hadron Collider beauty (LHCb) experiment, the Large Hadron Collider forward (LHCf) experiment and the TOTal Elastic and diffractive cross section Measurement (TOTEM) experiment. ALICE, ATLAS, CMS and LHCb are installed in four huge underground caverns built around the four collision points of the LHC beams. TOTEM will be installed close to the CMS interaction point and LHCf will be installed near ATLAS.

- ALICE is a detector specialised in analysing lead-ion collisions. It will study the properties of quark-gluon plasma, a state of matter where quarks and gluons, under conditions of very high temperatures and densities, are no longer confined inside hadrons.

- ATLAS and CMS are general purpose detectors designed to cover the widest possible range of physics at the LHC, from the search for the Higgs boson to supersymmetry (SUSY) and extra dimensions.

- LHCb specialises in the study of the slight asymmetry between matter and antimatter present in interactions of B-particles (particles containing the $b$ quark).

- LHCf is a small experiment that will measure particles produced very close to the direction of the beams in the proton-proton collisions at the LHC. The motivation is to test models used to estimate the primary energy of the ultra high-energy cosmic rays.

- The TOTEM experiment and its detectors will be discussed in Chapter 2 .

\subsection{Scope}

The TOTEM experiment will measure the total proton-proton cross section and studies elastic scattering and diffractive dissociation at the LHC. To collect data from the detector area, to build a consistent event frame and to send it to the counting room 1 , the TOTEM requires the hardware able to meet the detector bandwidth requirements. The FPGA devices seem to be the best choose for such applications. They allow:

— for parallel data processing;

- for arbitrarily data flow;

- for flexible logic modification of the already implemented system;

- finally, their timing capabilities meet the requirements of the DAQ system.

The third attribute is particularly crucial for the physics experiment, which is still in the commissioning phase, such as TOTEM, when the firmware constantly needs upgrades and new features.

The TOTFed motherboard is the main part of the on-line Data Acquisition System in the TOTEM experiment (Chapter 3). It divides the work between 7

\footnotetext{
${ }^{1}$ where the collisions events are reconstructed
} 
FPGAs and uses specialized interfaces such VME64x, S-Link 64, Gigabit Optical Link (GOL) to accomplish the objectives. This thesis focuses on the firmware of the programmable devices, which author designed and implemented and which are reported in Chapters 4,5 and 6.

Initially, the TOTFed was not able to meet the trigger requirements of $1 \mathrm{kHz}$ - the data rate was limited by about $12 \mathrm{MB} / \mathrm{s}$, which approximately corresponds to the frequency of $500 \mathrm{~Hz}$ of the trigger. Two out of three groups of FPGA devices installed on the board, namely the VME and the Main FPGA, used the .bdf $2^{2}$ format. Their internal structure was described by a network of logic gates, multiplexers and flip-flop blocks $s^{3}$. The author was asked to improve the TOTFed firmware and provide a reliable system able to send consistent data from the detector area with speed of about $20 \mathrm{MB} / \mathrm{s}$.

In the beginning, all the designs were converted to the VHDL format 4 . First of all, it allowed for the use of popular EDA tools, such as the ModelSim 5 to build the testbenches. Secondarily, since the .vhd format is based on text files, the SVN versioning system could have been used, which allowed for concurrent development of the same piece of code by several people. Moreover, the VHDL description is at higher level of abstraction than in the .bdf. Consequently, the designed solutions are still legible even if they are quite complex. The author imposed a structure of the top level entities 6 , in which each group of FPGA chips was assigned a specific package with a set of constants and settings. This approach simplified management of the projects.

First serious problem of the TOTFed was the communication between FPGAs. During the initial months of the author's work, he entirely designed and implemented the Local bus slave controller (Section 5.2.1) and the Opto-local bus (Section 6.2.2. Further tests indicated that the VME Controller needed to be change as well. For this reason the author redesigned completely the logic, including the FSM, leaving only the original implementation of the internal registers (Section 4.2.3). On this occasion, he contributed to the design of the TOTFed memory space (Section 3.3.

After the internal communication started to work correctly, the work effort was switched to the data transmission from the GX receiver to the VME bus. The author, together with Michele Quinto, designed the Data Processing (Section 6.2.5) and the OptoRx receiver (Section 5.2.2). He also took part in testing of the Data emulator of the OptoRx (Section 6.2.1) and fixed the bugs related to clock domains in the TTCrx Receiver (Section 6.2.3).

The subsequent tests revealed the reset problem of the motherboard. For this reason, the author designed and implemented the uniform reset system of the TOTFed (Section 3.4) and the VME Reset Subsystem (Section 4.2.2).

Finally, it became necessary to support the I2C communication with the TTCrx chip. This gave the author possibility to write and debug the I2C_Controller (Section 4.2.1.

\footnotetext{
2 Block Design File

3 exceptions was the VME Controller (Section 4.2.3

${ }^{4}$ Very High Speed Integrated Circuits Hardware Description Language

5 a digital ASIC simulation and verification program

${ }^{6}$ also for the OptoRx
} 


\section{The TOTEM}

\subsection{Experiment overview}

The TOTEM (TOTal Elastic Scattering and Diffraction Measurement) [1] [2] experiment will measure the total proton-proton cross section and will study the elastic scattering and diffractive dissociation at the $\mathrm{LHC}^{1}|3|$. More specifically, TOTEM focuses on:

- the total cross-section with an absolute error of $1 \mathrm{mb}^{2}$ by using the luminosity independent method. This requires the simultaneous measurement of the elastic pp scattering down to the four-momentum transfer of $-t \approx 10^{-3} \mathrm{GeV}^{2}$ and of the inelastic pp interaction rate with an adequate acceptance in the forward region;

- elastic proton scattering over a wide range in momentum transfer up to $-t \approx 10 \mathrm{GeV}^{2}$;

- diffractive dissociation, including single, double and central diffraction topologies using the forward inelastic detectors in combination with one of the large LHC detectors.

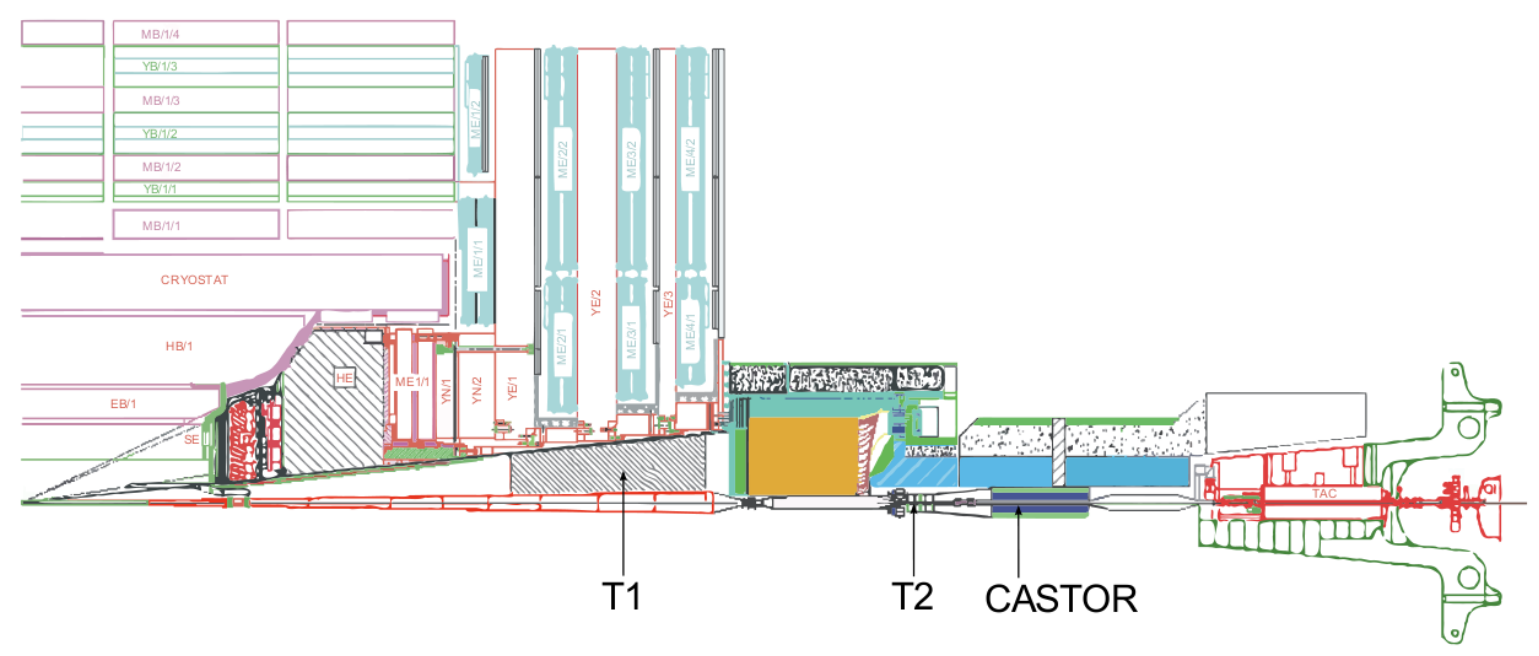

Figure 2.1. The TOTEM detectors installed in the CMS forward region.

The TOTEM is the smallest experiment at the LHC hosted by the bigger one $\mathrm{CMS}^{3}$. From the technical point of view, it means, that data, trigger and commands

\footnotetext{
1 the Large Hadron Collider

2 mili barn, barn is a unit used in nuclear physics for expressing cross sectional area of nuclei. $1 \mathrm{~b}=10^{-28} \mathrm{~m}^{2}$ (size of an uranium nucleus) [4]

3 the Compact Muon Solenoid
} 
format should be compatible with the CMS detector. The experiment consists of two tracking telescopes T1 and T2 [5], and so-called "Roman Pots" placed on both sides of an interaction point where accelerated particles collide with the target $14 \mathrm{TeV}$ centre of the mass energy.

The nearest telescope to the interaction point is T1 $(z=9 \mathrm{~m})$. It consists of the Cathode Strip Chambers - CSC [1]. T2 exploits the Gas Electron Multipliers (GEM) [5] and is located further $(z=13.5 \mathrm{~m})$. The objective of the above two telescopes (Figure 2.1) is to detect and measure inelastically produced charged particles. To accomplished coverage in the pseudorapidity ${ }^{4}$ range of $3.1 \leq|\eta| \leq 6.5$ TOTEM is complemented by the Roman Pots (Figure 2.2) - detectors in special movable beam-pipe insertions, placed at about $147 \mathrm{~m}$ and $220 \mathrm{~m}$ from the interaction point. They are silicon devices designed by TOTEM to detect the leading protons at merely a few $\mathrm{mm}$ from the beam centre.

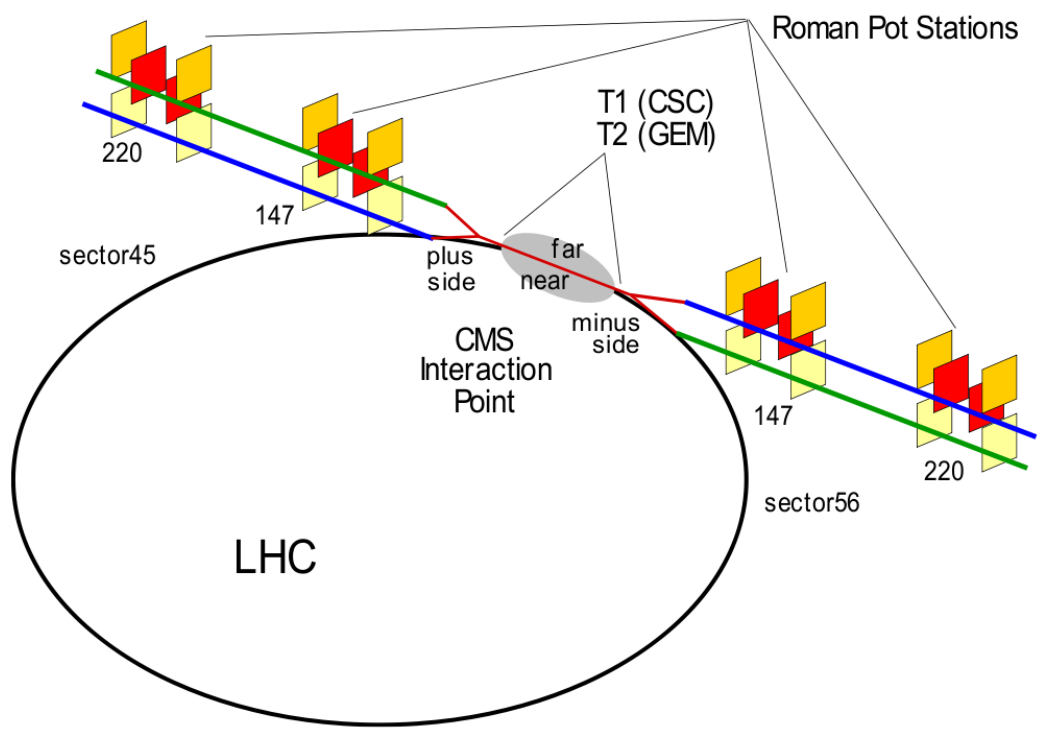

Figure 2.2. Location of the TOTEM Roman Pots at distances of about $147 \mathrm{~m}$ (RP147) and $220 \mathrm{~m}$ (RP220) on both sides of IP5.

\subsection{General overview of the TOTEM readout system}

Although each of the three subdetectors exploit distinct technology and physics principles to detect the particles, they adopt the same front-end readout chip called the VFAT (Very Forward ATLAS and TOTEM chip) - Section 2.5. This approach has the obvious benefit of reducing design effort by using unified electronic components, data format and software for Data Acquisition (DAQ). Table 2.1 presents utilization of the VFATs by each of subdetectors.

Figure 2.3 presents general scheme of the front-end electronic of the TOTEM. As the figure shows, it can be divided into a readout and a control path. However this document, as the author was engaged in a firmware development for the TOTFed,

\footnotetext{
4 "In experimental particle physics, pseudorapidity, $\eta$, is a commonly used spatial coordinate describing the angle of a particle relative to the beam axis. It is defined as $\eta=-\ln \left(\tan \left(\frac{\theta}{2}\right)\right) " \mid 6$

5 in rad unit: Radiation absorbed dose
} 


\begin{tabular}{|c|c|c|c|}
\hline & RP & T1 & T2 \\
\hline $\begin{array}{c}\text { No. and type } \\
\text { of detectors }\end{array}$ & $\begin{array}{c}240 \\
\text { Si Strip } \\
\text { Detectors }\end{array}$ & $\begin{array}{c}60 \\
\text { Cathode Strip } \\
\text { Chambers }\end{array}$ & $\begin{array}{c}40 \\
\text { Gas Electron } \\
\text { Multipliers }\end{array}$ \\
\hline No. of channels & 122880 & $\begin{array}{c}11124 \text { anodes } \\
15936 \text { cathodes }\end{array}$ & $\begin{array}{c}\text { 62400 pads } \\
20480 \text { strips }\end{array}$ \\
\hline No. of VFATs & 960 & 480 & 680 \\
\hline Typical input charge & $4 \mathrm{fC}$ & $50 \mathrm{fC}$ & $50 \mathrm{fC}$ \\
\hline Radiation dose 5 & $<10 \mathrm{Mrad}$ & $<50 \mathrm{krad}$ & $50 \mathrm{Mrad}$ \\
\hline
\end{tabular}

Table 2.1. Overview of electronics from different detectors.

will focus on the first one. The VFAT data are transmitted to Gigabit Optical Link chip (GOL) [7| hosted on Gigabit Optical Hybrid (GOH) where are transformed from an electrical into optical signals. Optical lines are collected into the OptoRx module, which makes revert signal transformation. As a result, after a relatively long way, electric signals are back on a counting room region.

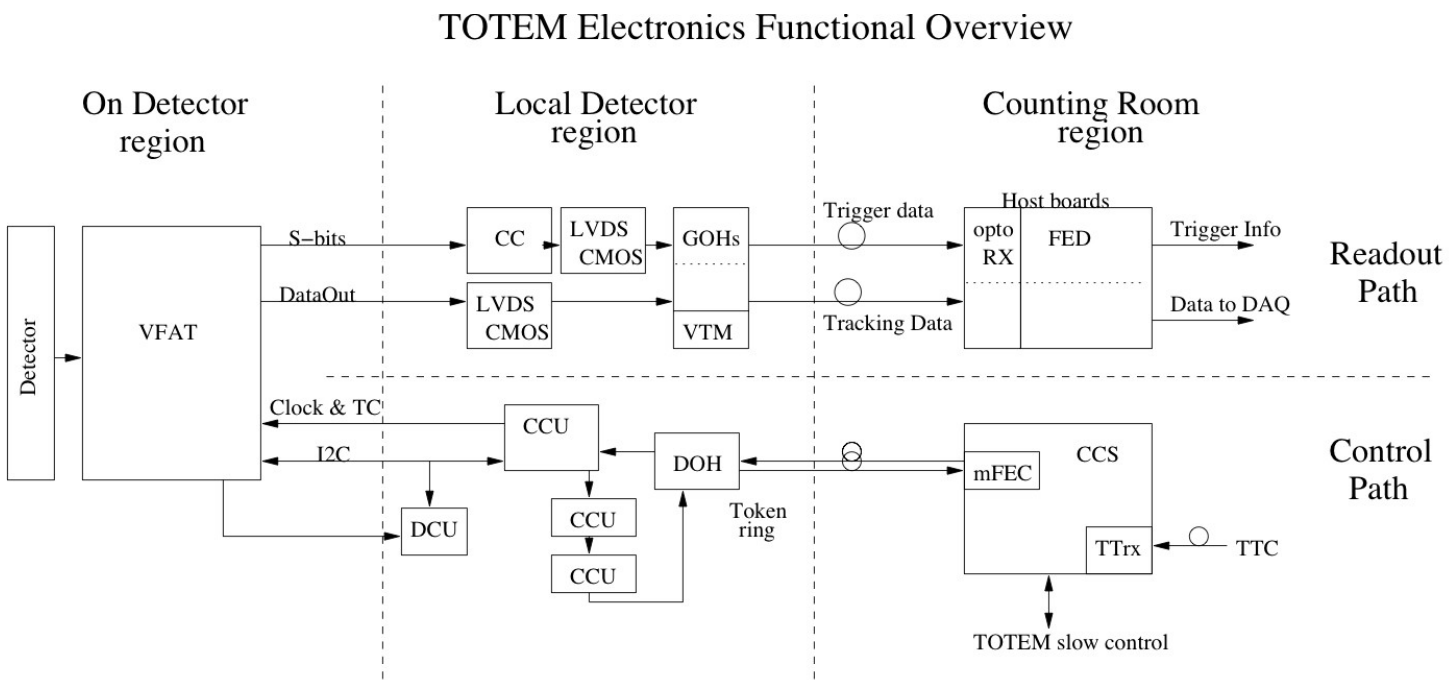

Figure 2.3. The scheme of the TOTEM's front-end electronics.

The OptoRx is housed, via a special connector, on the TOTFed ${ }^{6}$ board, equipped with FPGA devices to process and buffer data, to check consistency and send the prepared packets to the local DAQ. Each transition from the OptoRx to the central CMS or local DAQ has to be done through FED.

\subsection{TOTEM Local DAQ system}

\subsubsection{Architecture of the readout chain}

The readout chain of the TOTEM is presented in Figure 2.4. Each of the sixteen VFATs is connected electrically to a one GOH. Twelve GOHs create a bundle of optic fibres, that goes directly to a OptoRx on the TOTFed. The TOTFed collects

${ }^{6}$ in the CMS jargon is simply the FED (Front End Driver) 
data at most from three OptoRx devices and builds an event frame which contains all tracking information from detector region serviced by the TOTFed. The data are buffered on the motherboard and wait for a readout via VME (Versa Module Eurocard) $[8]$. When it initiates, data are sent to a group of local PCs, that filter and store information.

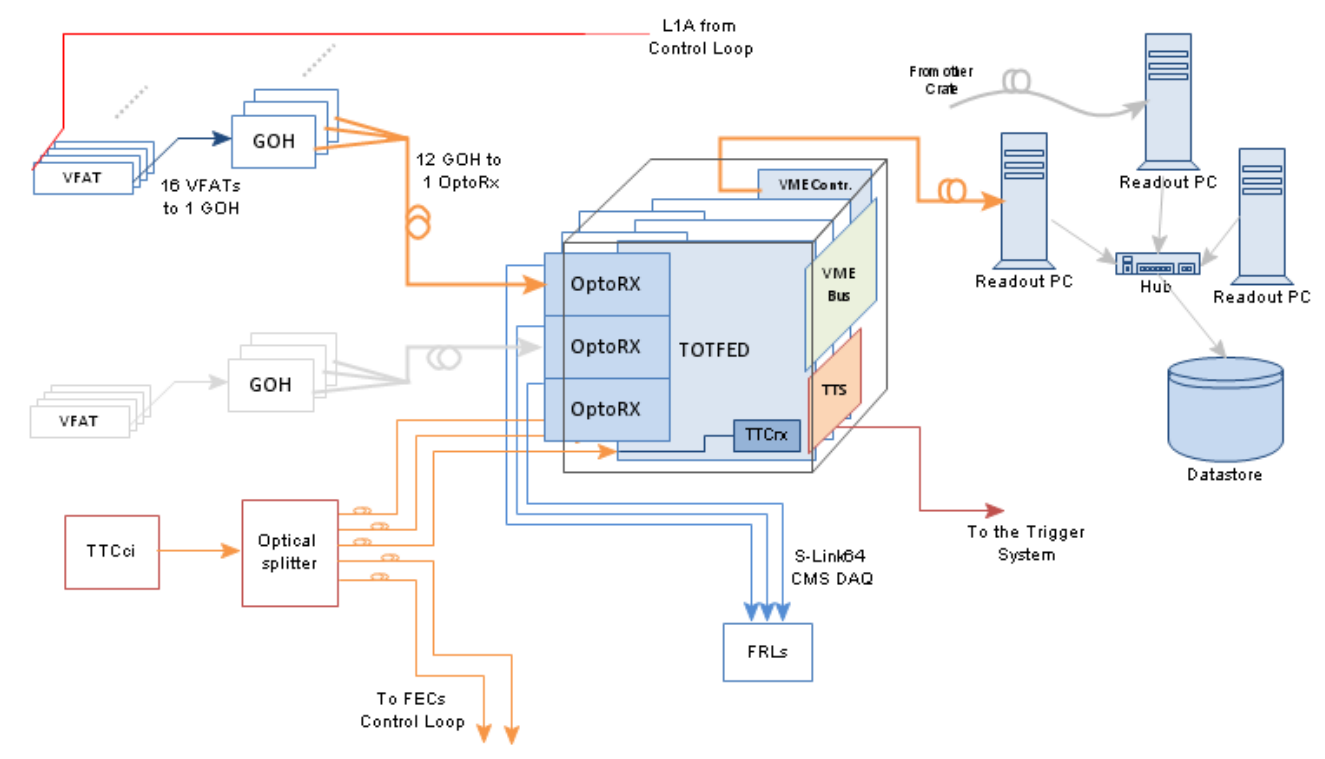

Figure 2.4. General scheme of the DAQ architecture.

The data flow starts when the VFATs receive a trigger signal - Level One Accept (L1A). This signal is generated by dedicated system called Timing, Trigger and Control CMS interface (TTCci) [9|. The L1A is provided to the VFATs by the Control and Clock System (Section 2.3.2 ), which, roughly speaking, translates the TTC information to simple electric signals.

\subsubsection{The Control and Clock System}

Controlling of VFATs is made by a special ring architecture, called the Control and Clock System (CCS). The first element on the control path is FEC (The Front-End Control) $[10]$ board. It is capable to serve up to eight rings, using dedicated mezzanines - mFECs. General scheme of the FEC is presented in Figure 2.5. The TTC signals are received by a board and translated to the protocol of the ring. Using the FEC, operator is able to set complex trigger conditions. What is important, FEC supports the TTS (The Trigger Throttling System) (Section 2.3.4) - an internal logic, which can slow down the trigger rate, if the DAQ system is overloaded.

The control ring consists of the mFEC and CCU devices (Communication and Control Unit). As it is presented in Figure 2.6, the CCU supports many simple protocols. Between the CCUs a cabling redundancy can exist to let the system work, even if one of the components is broken (the ring will simply omit it). VFAT, as it is done in the TOTEM experiment, can be one of the CCU's slaves. 


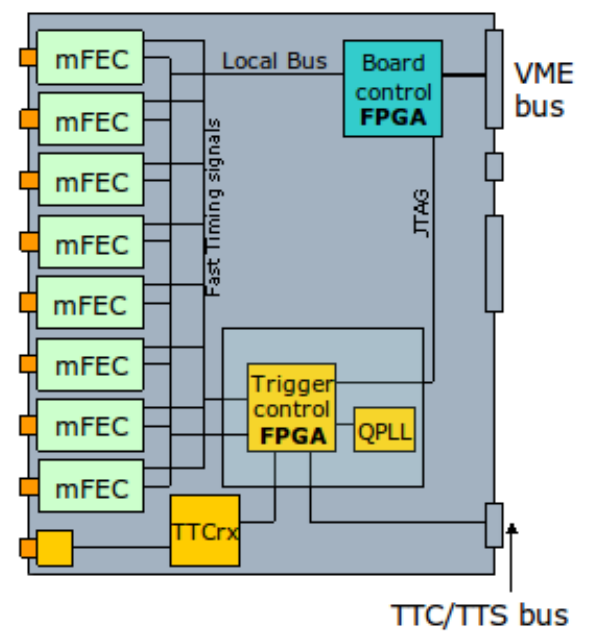

Figure 2.5. Genereal scheme of FEC.

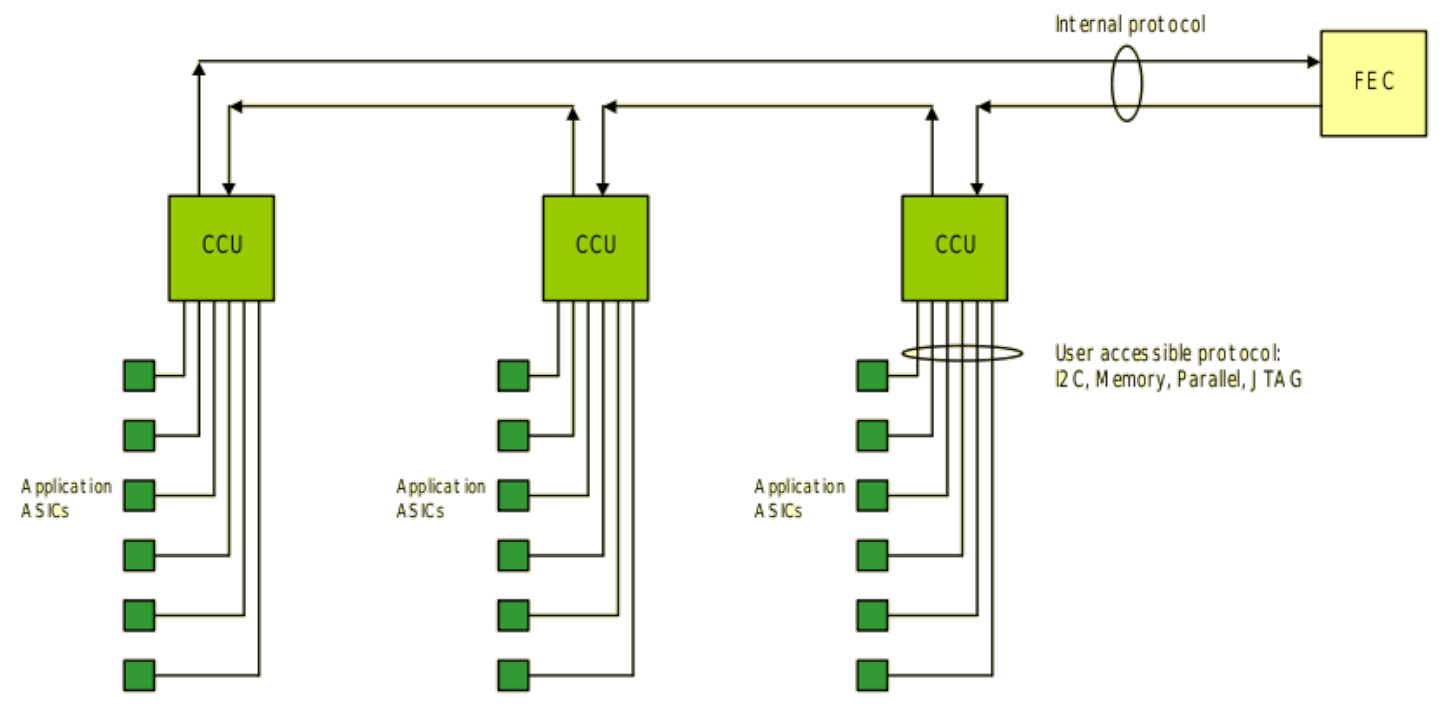

Figure 2.6. Architecture of the ring in CCS.

\subsubsection{Timing, Trigger and Control System}

The TTCci (Figure 2.4) is a very important block from the DAQ point of view. As it was mentioned before, the TTCci provides the L1A signal. However, this component delivers much more information to the control and readout chain:

- the LHC machine global clock;

- the LHC orbit;

- the first level trigger: Level One Accept (L1A);

- the fast commands, that can be used to reset, control and calibrate the readout chips (VFATs).

As delay and crosstalk of timing signals are critical for the TOTEM, TTCs lines are distributed directly to each TOTFed card and the CCS by an optical link. It forces the usage of additional optical splitters. 


\section{TTC Receiver Chip}

As shown in Figure 2.4, every TOTFed is equipped with a Timing, Trigger and Control receiver (TTCrx). The TTCrx is an ASIC chip designed at CERN, that receives and decodes TTC signals to generate internally the timing data:

- the event counter, that represents the number of transmitted L1As;

- the event reset;

- the bunch crossing counter, that can be considered as the event time stamp;

- the bunch crossing zero (BCO) signalling the LHC orbit.

On the TOTFed, information from TTCrx are independently available for the VME and the three OptoRxs.

\subsubsection{Trigger Throttling System}

The feedback from the front-end electronics to the trigger source is assured by so-called Trigger Throttling System (TTS). This system has defined binary statuses (Table 2.2), that can be changed by the trigger logic (for example in a FEC) to adjust the trigger frequency or ask for resynchronization in case of system errors.

As the TOTEM is hosted by the CMS, it has to follow a well defined protocol, common to all CMS subdetectors serviced by the DAQ of CMS. It is obligatory, to implement the TTS state machine with a hysteresis mechanism to avoid neighbour state oscillations (digital hazards). The TTS state-transition diagram is presented in Figure 2.7.

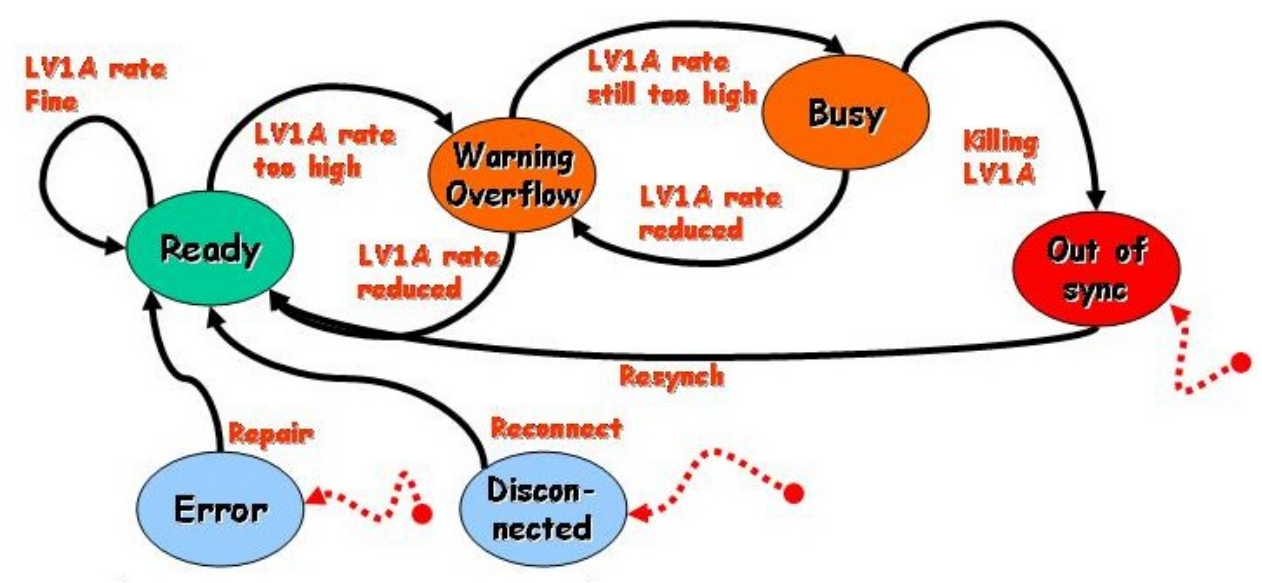

Figure 2.7. The TTS state-transition diagram.

Since the TOTEM experiment is still in the initial phase and not yet fully combined with the CMS, the TOTFed works in a local mode. It means, that at the moment, the motherboard uses simply the busy state, to make the TOTEM trigger system aware of buffer-full condition and out of synch to mark suspicious events in the DAQ. 


\begin{tabular}{c|c|l} 
Value & State & Comment \\
\hline 0000 & Disconnected & Hardware failure or broken cable \\
0001 & Warning overflow & Imminent buffer overflow \\
0010 & Out of synch & FED is no more synchronized with the TTC values \\
0100 & Busy & Cannot accept triggers \\
1000 & Ready & Ready to accept triggers \\
1100 & Error & Any other state that prevents correct functioning \\
1111 & Disconnected & Hardware failure or broken cable \\
others & & Reserved
\end{tabular}

Table 2.2. TTS state machine coding.

\subsection{The CMS Common Data format}

Because of the fact, that TOTEM works as a part the CMS DAQ, the readout requires to use the special data format called "CMS common data format". Every transmitted piece of data is encapsulated by hardware in to a packet of 64-bit words. Data are transmitted to a storage using the S-Link64 interface ( $|11|)$. Figure 2.8 presents structure of the packet.

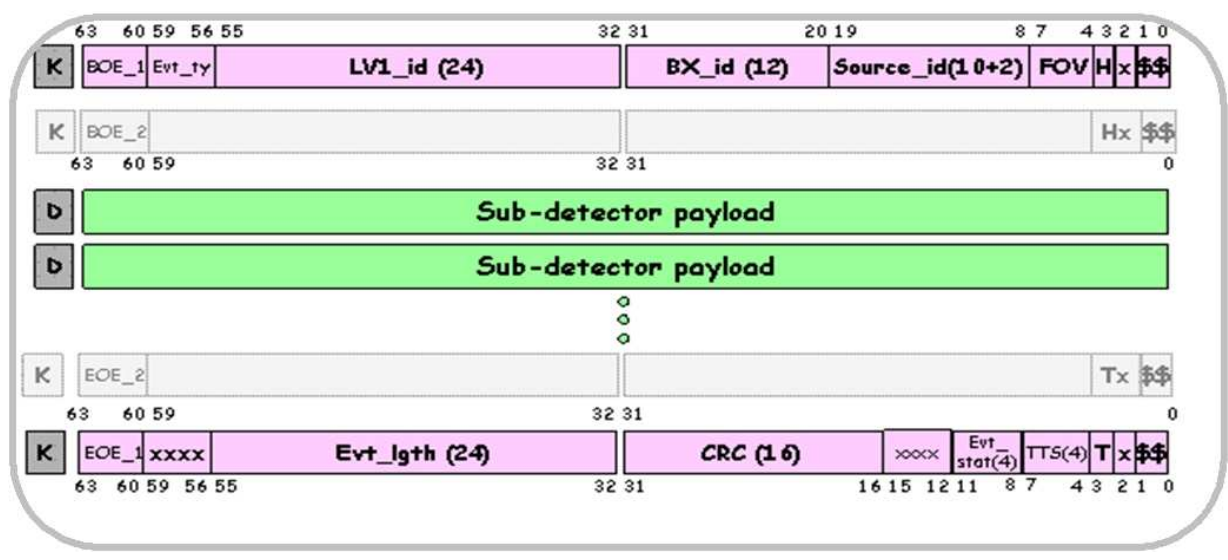

Figure 2.8. CMS Data Format.

The headers and trailers are added by a S-Link sender (i.e. the OptoRx) and contain fields to simplify the management of the events collected by the experiment. Their meanings is the following:

— BOE_n: the identifier for the beginning of an event frame $\left(\mathrm{BEO}_{-} 1=0 \times 5\right)$.

- Evt_ty: the event type identifier.

- LV1_id: the level-1 event number generated by the TTC system.

- BX_id: the bunch crossing number. Reset on every LHC orbit.

- Source_id: unambiguously identify the data source (FED/DCC).

- FOV: version identifier of the common FED encapsulation (header+trailer).

- H: when set to 'O', the current header word is the last one. When set to ' 1 ', another header word is following.

- EOE_n: the identifier of the end of an event fragment (EOE_1 $=0 x A)$.

- Evt_lgth: the length of the event fragment counted in 64-bit words including header and trailer. 
- CRC: Cyclic Redundancy Code of the event fragment including header and trailer.

- C : when set to ' 1 ', the FRL has detected a transmission error over the S-link cable. The FED/DCC must set this bit to 'O'.

- F : when set to '1', the FED_ID given by the FED is not the one expected by the FRL. The FED/DCC must set this bit to 'O'.

- Evt_stat: the event fragment status information.

- TTS: current values of the TTS bits.

- T: when set to '0', the current trailer word is the last one. When set to ' 1 ', another trailer word is following.

- R: when set to ' 1 ', the CRC value has been modified by the S-link sender card. The FED/DCC must set this bit to 'O'.

- $\mathbf{x}$ : indicates a reserved bit. The FED/DCC must set this bit to 'O'.

- \$: indicates a bit used by the S-LINK64 hardware. The FED/DCC must set this bit to 'O'.

The CRC code is calculated according to the CRC-16CCITT specification. The ready VHDL code is provided and suggested to use by authors of the "CMS common data format" [12]. The CRC field, should force all bits of the calculated checksum to 'O'.

\subsection{VFAT}

The first level of the TOTEM's DAQ is the VFAT (Very Forward ATLAS and TOTEM chip) [13|. It is a trigger and tracking front-end ASIC, designed specially for the readout of sensors in the TOTEM experiment at the LHC. Figure 2.9 shows how the VFAT looks like.

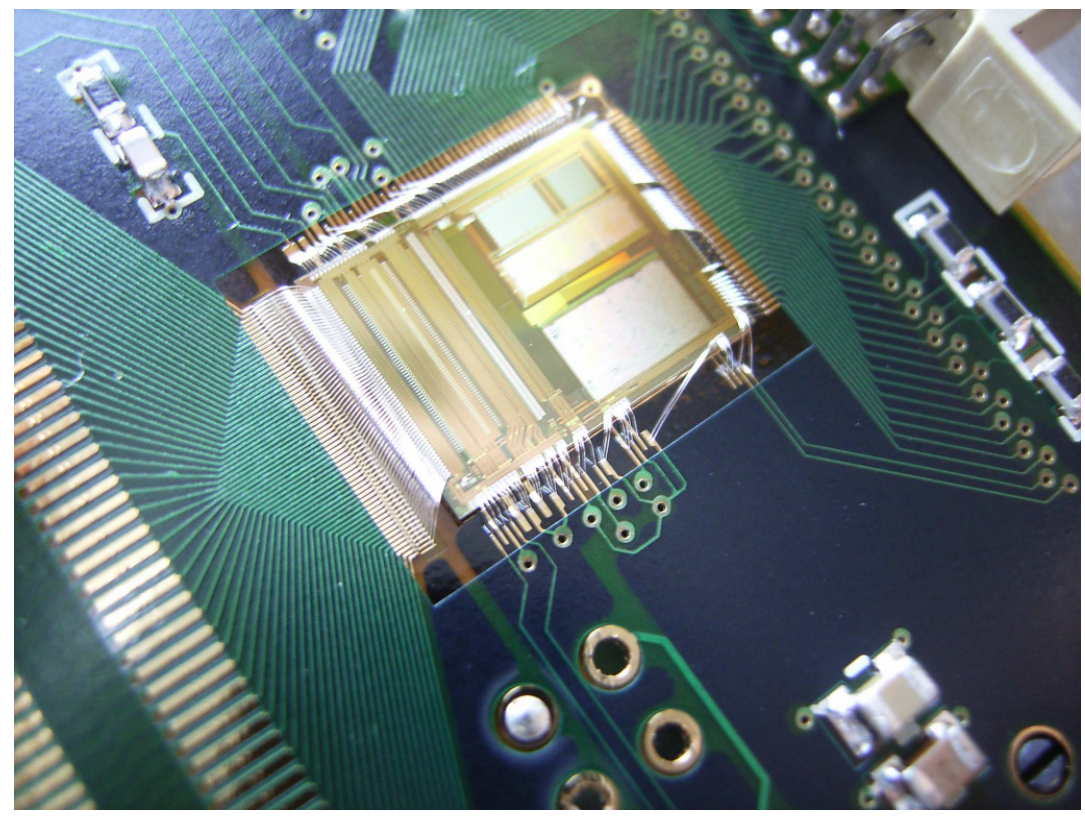

Figure 2.9. Photograph of the VFAT chip.

The VFAT device has the two following functions: 
— tracking — provide spatial hit information about triggered event;

- trigger - provide programmable "fast OR" function, that is used to create a level-1 trigger (L1A)

\subsubsection{General description}

The VFAT is a device, that combines analogue and digital part of the experiment. The analogue front-end detects and converts the electric charge from 128 inputs, while the digital part generates the data frame to be transmitted to the readout system (Figure 2.10). The digital parts uses a general LHC system clock of frequency $40.08 \mathrm{MHz}$, that synchronize all the detectors of the LHC.

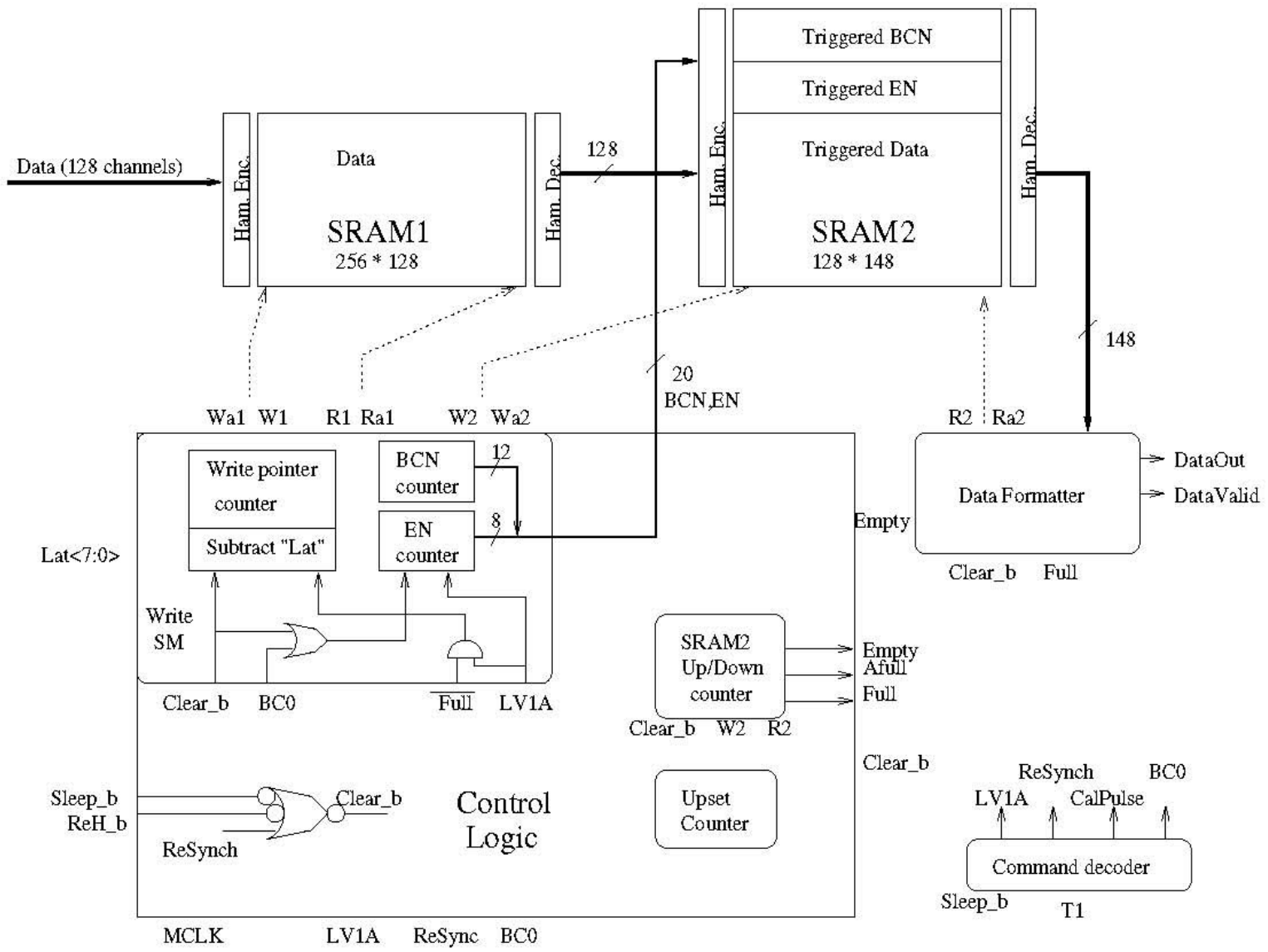

Figure 2.10. Scheme of the VFAT.

The data flow starts, when the VFAT receives the L1A trigger signal. The internal state machine extracts the event from the SRAM1 and combines it with the time stamp (BCN) and the event counter (EN) in SRAM2. At the end, data pass by a data formatter where CRC checksum is calculated, and finally are transmitted serially to a next level of the DAQ. The output frame is indicated by a signal Data Valid (DV). 


\subsubsection{The VFAT data format}

The VFAT data frame contains 128 bits, which represent charge detected from 128 channels of a subdetector ${ }^{7}$ encapsulated by header and trailer. It means, that a single frame counts 192 bits (Figure 2.11).

The frame of the VFAT is accordingly composed of:

1. Header:

a) The Time Stamp, from the MSB to the LSB: a 4 bit identifier 1010, followed by 12 bits of the actual time stamp (BC);

b) The Event Counter, from the MSB to the LSB: a 4 bit identifier 1100, followed by 8 bits of the actual event counter (EC) and 4 bits for VFAT's flags;

c) The Chip ID, from the MSB to the LSB: a 4 bit identifier 1110 , followed by 12 bits of the hardware chip identifier.

2. Payload -128 bits of data from the detector

3. Footer - 16 bits of the CRC checksum (Cyclic Redundancy Check code). It is calculated according to the CRC-16-CCITT 8 specification excluding the VFAT's trailer.

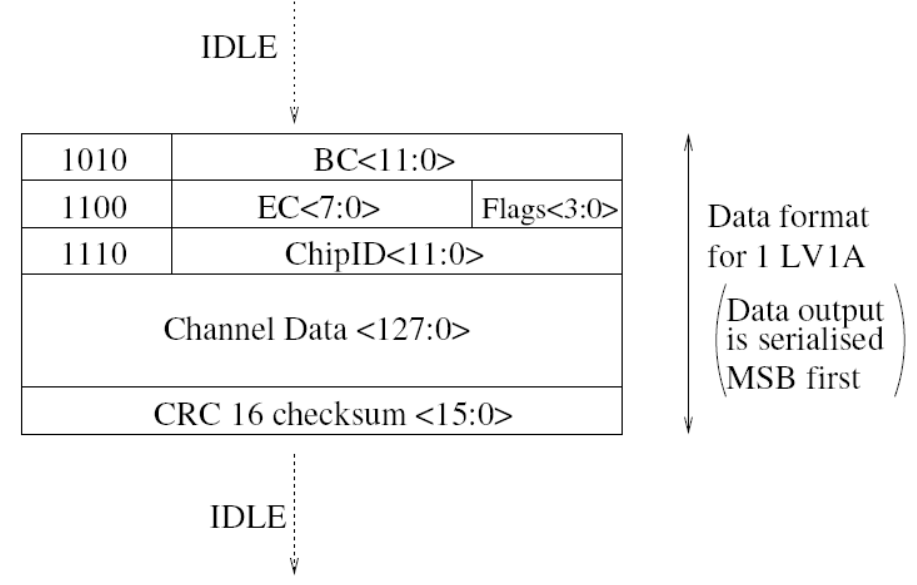

Figure 2.11. The VFAT frame.

\subsection{GOH}

The GOH (Gigabit Optical Hybrid) is the last part of the readout system in the interaction area. It converts the electrical data from the VFATs to an optical form and sends it to the OptoRx. The GOH uses the GOI (Gigabit Optical Link) |7| input chip.

The GOL, which in fact can be considered as a data serializer, enables to use different transmissions protocols. For the TOTEM readout purpose it has been configured in Gigabit Ethernet mode (8b/10b encoding). Up to 16 VFAT chips are

\footnotetext{
7 The single bit represents a single hit on the fragment of the detector connected to input channel.

${ }^{8}$ Polynomial form: $x^{16}+x^{12}+x^{5}+1$. Very popular kind of the CRC checksum used by XMODE, Bluetooth, SD, etc.

9 an ASIC chip designed at CERN
} 


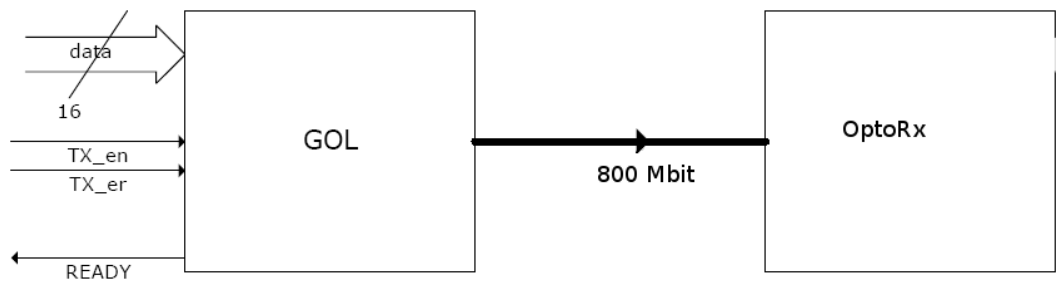

Figure 2.12. Data chain in the GOH.

connected to the GOL input channels (Figure 2.12). One of the VFATs is defined as a master and controls the GOL input called Transmission Enable (TX_en). Once the GOL is locked and initialized it starts sending an idle control pattern (0xBC50 or 0xBCC5) to synchronize a receiver. As it is shown in Figure 2.13, the data are sent, when TX_en input signal is asserted.

\begin{tabular}{|c|c|c|c|c|c|c|c|c|c|c|}
\hline \multicolumn{3}{|c|}{ IDLE pattern } & SOF & \multicolumn{5}{|c|}{ Data Packet } & \multicolumn{2}{|c|}{ IDLE pattern } \\
\hline IDLE & IDLE & IDLE & CXT & DATA & DATA $\cdots \cdots$ & DATA & DATA & CRC & IDLE & IDLE \\
\hline
\end{tabular}

Figure 2.13. Data packet from GOH.

\subsection{Experiment configuration}

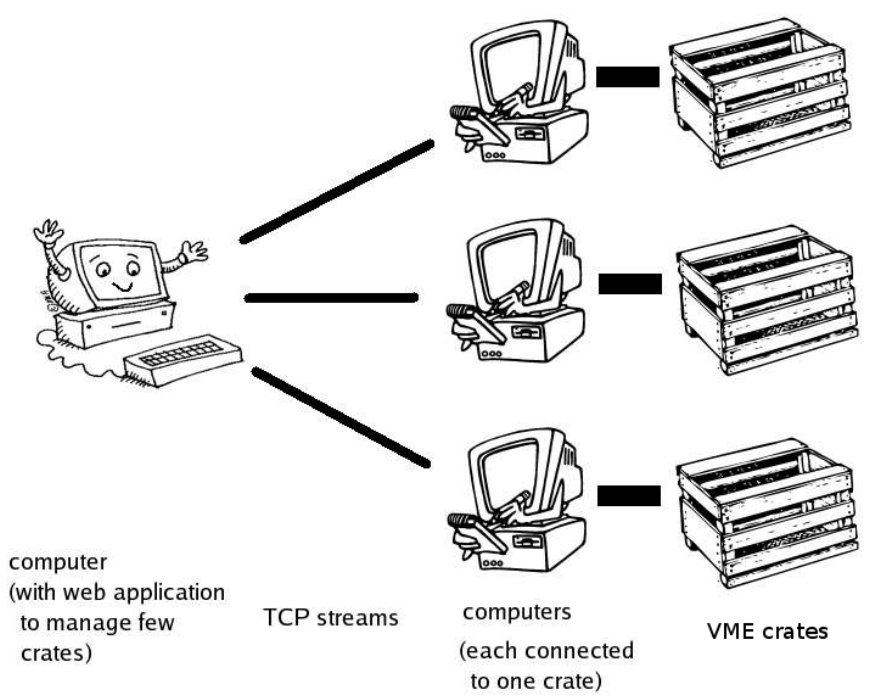

Figure 2.14. Schema of the readout system in DAQ.

Since TOTEM uses three various subdetectors to detect particles, there is a need to use three separate VME crates to read data in the DAQ. As it is presented in Figure 2.14, each VME crate responsible for the data readout from a single subdetector, is assigned to a separate computer. The computers control the VME buses 
using the special PCI-VME optical link bridges - V2718 [14 provided by CAEN company. The producer includes the Unix library, which provides a framework in $\mathrm{C}++$ language to control the crates.

All computer communicate through TCP protocol to a master machine, which controls the whole system. A single VME crate consists of:

- VME 64 bus driver, this is the master of the local VME bus controlled by an assigned computer;

- TTCci, to receive the TTC signals and generate the L1A;

- FEC, to control the the CCS (Section 2.3.2) loop;

- two TOTFeds, to collect data from the subdetector.

The TOTEM experiment uses one common LTC (Local Trigger Controller) to receive clock and orbit signals from the LHC. It allows to operate in a stand-alone mode using the local DAQ and the local triggers independently of the CMS. Moreover, the module is capable to receive the TTS signals (Section 2.3.4) ensuring the readout overload protection. The LTC controls TTCci boards installed in every crate. The TOTEM has also an additional VME crate which contains the trigger TOTFeds. Their objective is to provide the trigger for the experiment, basing on current data from the detector ("fast or" function of the VFATs - Section 2.5). The trigger is routed to the LTC and then, depending on the current TTS state, it makes a decision about the L1A generation. 


\section{The TOTFed}

\subsection{General Overview}

The TOTFed is a ten-layer motherboard designed according to the VME specification. The size of the board is $402 \times 366.7 \mathrm{~mm}$ - it is the biggest one (9U) provided by the standard. Figure 3.1 presents the TOTFed motherboard.

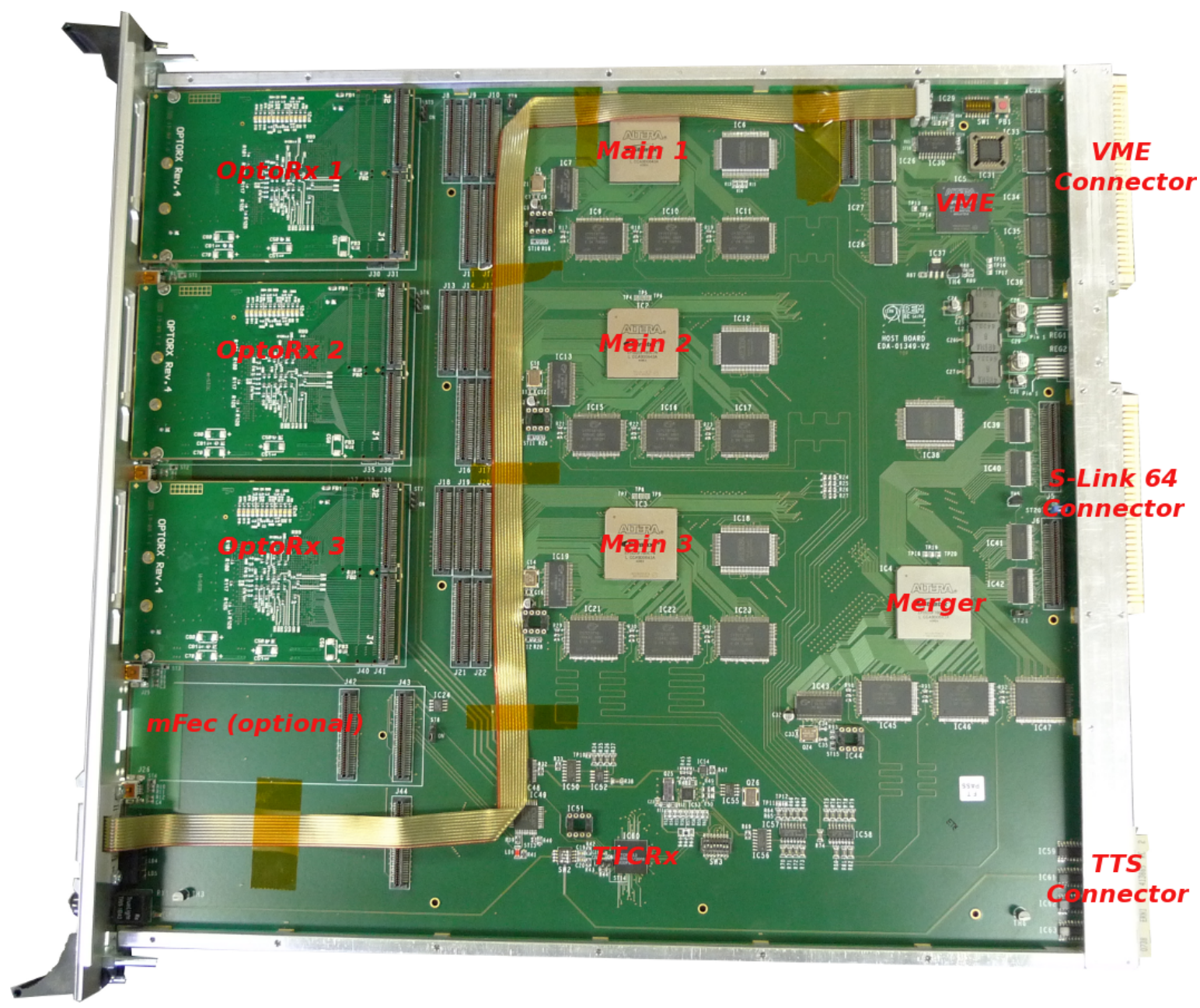

Figure 3.1. The photograph of the TOTFed motherboard with three OptoRx mezzanines connected.

The author would like to point out, that the TOTFed was not only designed for the TOTEM experiment. It is a reference board used as well by the CMS project. In addition, the functional requirements and the planned usage of the 
board resources changed during the design process. It explains, why not all the components are used at the current state of the project and why some non-optimal solutions were required, which could have been simply solved at the board-design stage, to avoid the complex firmware in some parts.

The scheme of the TOTFed is presented by Figure 3.2. Each OptoRx, where a data flow starts, is connected directly to the associated Main via two separate data buses: S-Link 64 bus, and Opto-local bus. The S-Link 64 bus is a local implementation of the interface, which allows to send 64 bits of data in a single word. Data are sent in one direction, from the master - an OptoRx, to the slave a Main, clocked with $40 \mathrm{MHz}$ clock. In the future, the S-Link 64 bus will be routed to an output connector and passed directly to a storage device in the counting room. The Opto-local bus (16 bits) is used to configure the FPGA from the VME level and read the device status.

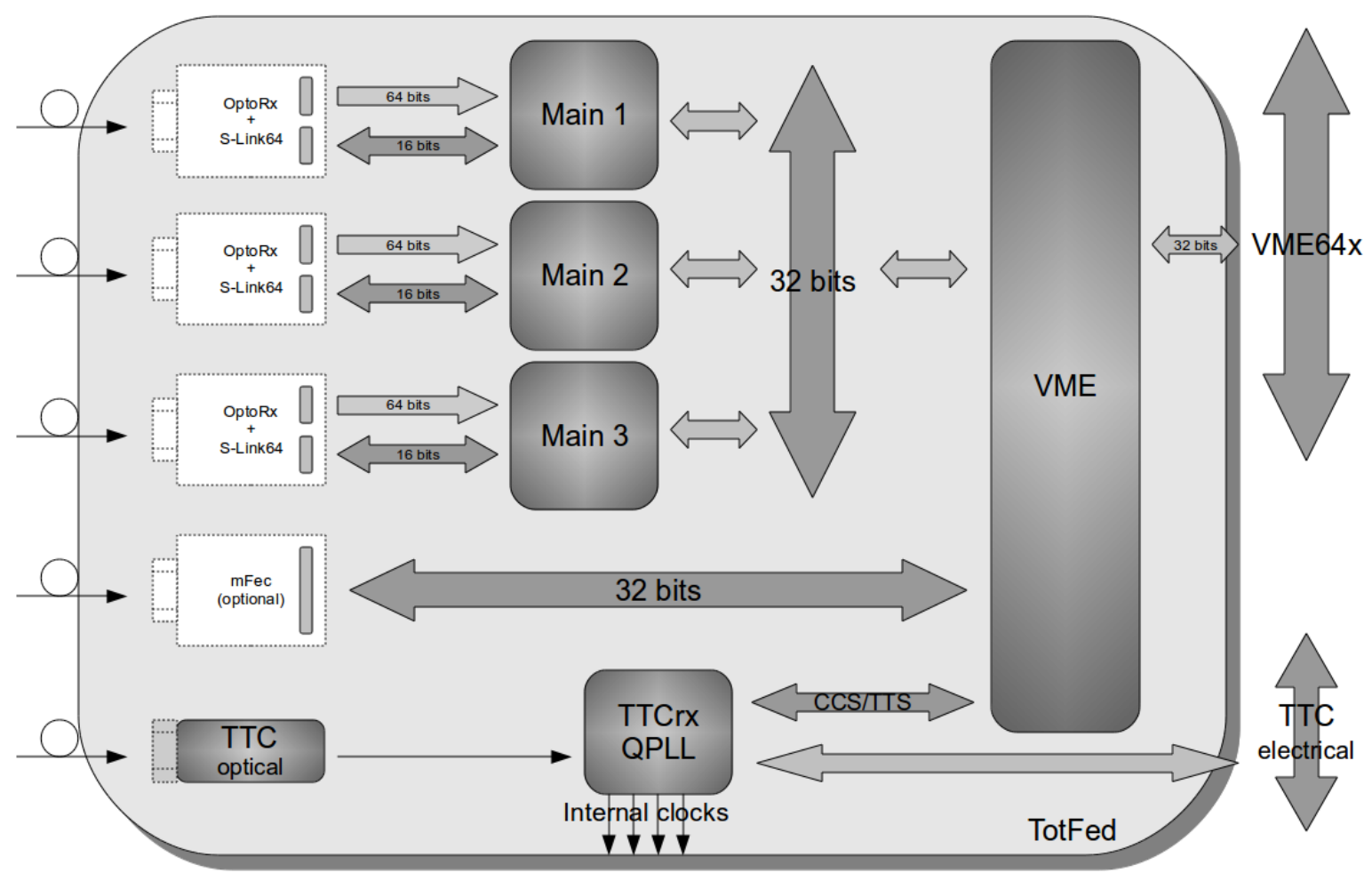

Figure 3.2. The scheme of the TOTFed.

Between the Mains and the VME there exists a shared 32-bit bus named Local bus controlled by the VME FPGA. It is used to read data from the VFAT chips as well as to configure and obtain the status of the TOTFed components.

The VME and the optional mFEC connector are linked together by a separate bus of 32 bits, which is used by the Trigger version of the TOTFed and is not covered by this document. However, it is possible to extend the Local bus with an additional device on the $\mathrm{mFEC}$ connector by writing a specific value to one of the registers of the VME.

The author took part in implementing and designing the firmware of the VME FPGA (Chapter 4), the Mains FPGAs (Chapter 5) and the OptoRxs FPGAs (Chapter 6), which is the scope of this thesis and is reported in the following chapters. 


\subsection{TTC signals distribution on the TOTFed}

The bottom of the Figure 3.2 presents the TTC path. An optical signal containing TTC information (Section 2.3.3) goes to the TTCrx. Decoded commands are sent independently to the VME and the three OptoRxs. The TTCrx provides as well 40 MHz reference clock to the QPLL (Quartz Crystal Based Phase-Locked Lop) [15]. The QPLL is an ASIC chip, that act as a jitter-filter and a clock multiplier for the LHC bunch-crossing frequency $(40.08 \mathrm{MHz})$. It is conceived to meet the LHC radiation requirements. The QPLL is a source of a clock signal for all synchronous device hosted by the TOTFed : the three OptoRxs, the three Main, the Merger 1 and the VME. The electrical paths to the Mains are specially tuned to obtain a coherent signal. The other devices are nor equipped with such a functionality. Although the GPLL is able to multiply the clock frequency, due to a physical limitations of the motherboard, the distributed clock signal frequency is $40.08 \mathrm{MHz}$. However, all FPGAs are equipped with internal PLLs so it is possible to generate higher frequency inside the programmable devices.

\subsection{Memory Space}

For the TOTEM purposes, the VME bus uses 32-bit words for both addressing and data [16]. Figure 3.3 presents the address mapping scheme.

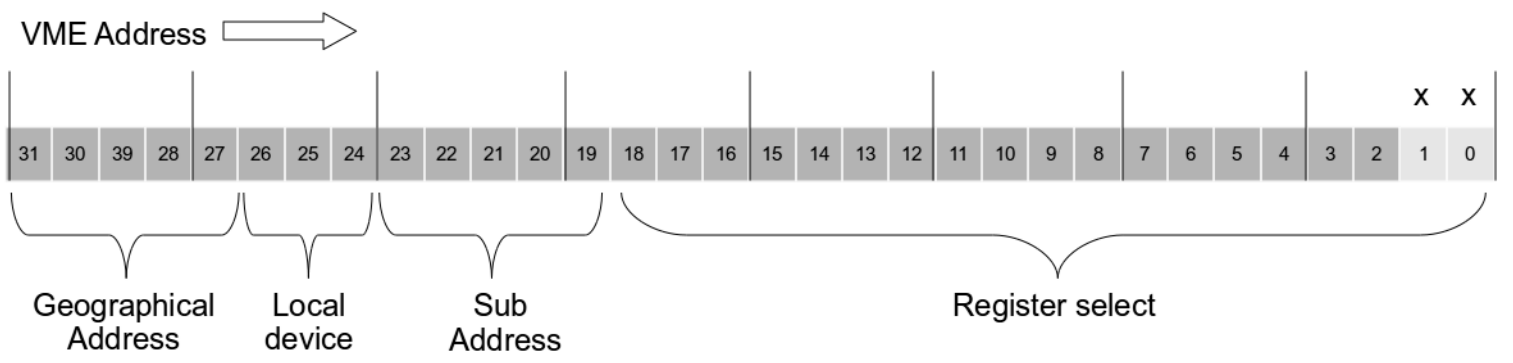

Figure 3.3. The address mapping scheme on the TOTFed motherboard.

The five most significant bits represent geographical address of the TOTFed. This is a hexadecimal code of a slot number, assigned by the VME bus independently for each of the slave devices, shifted left by 27 positions (i.e. for slot 1 memory space starts from 0x08000000). Next three bits, from the MSB to the LSB, designate device on the Local bus as it is presented in Table 3.1 (Local Device).

The subaddress part (Figure 3.3) is used to divide the FPGA device address space into memory pages reserved to slave devices of FPGAs (i.e. external memory and OptoRx). The remaining 20 bits represent registers addresses. The 2 least significant bits are not used, because of the chosen addressing mode (32 bits). For example, by switching to one on positions 20th and 19th of the VME address, it is possible to select the OptoRx card through the Main FPGA; i.e: if the TOTFed board is plugged into the 14th slot and one OptoRx is connected to the Main 1, the OptoRx 1 address space stretches from 0x 71180000 to 7118 03FF.

\footnotetext{
${ }^{1}$ The FPGA used in Trigger version of the TOTFed
} 


\begin{tabular}{c|c} 
Local Device & Assigned local device \\
\hline 0 & VME \\
1 & Main 1 \\
2 & Main 2 \\
3 & Main 3 \\
4 & Reserved - not used \\
5 & mFec connector \\
6 & Merger \\
7 & Reserved - not used
\end{tabular}

Table 3.1. Mapping of the local devices in the address

\subsection{Reset system of the TOTFed}

The reset subsystem scheme of the TOTFed is presented in Figure 3.4. All reset signals are distributed by the VME. Three ports act as the reset source. Two of them: the VME system reset and the TOTFed reset button are low-level, which means that through 'and' gate ${ }^{2}$ are routed directly to the outputs: board reset, QPLL reset and TTCrx reset. In contrast to them, the last source is synchronous and trigged by a write access through the VME bus to a dedicated register of the VME FPGA (address: 0x00000010). Whatever value is written to the register it enables a dedicated reset subsystem at the device.

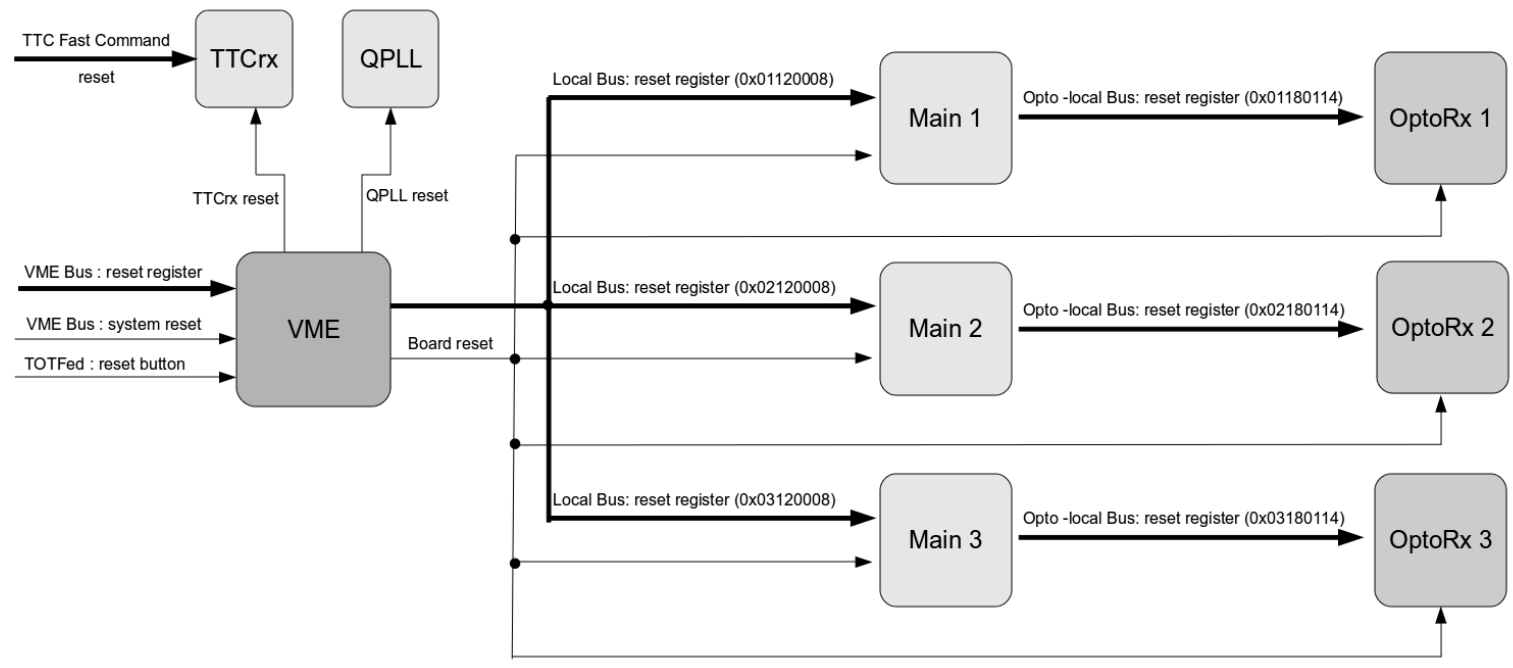

Figure 3.4. The reset subsystem scheme of the TOTFed.

The subsystem, as it resets the QPLL and consequently the clock source, uses the VME system clock of the frequency of $16 \mathrm{MHz}$ provided by the VME bus. The clock is needed by an internal counter, which measures the time of a reset pulse. According to the TTCrx manual [17], the width of the reset pulse depends on the pull-up and pull-down resistors located on the data bus between the TTCrx and the Mains. The resistors specify an ID of the TTCrx. In case of the TOTFed, the pulse should be about 500 ns long. It is routed through 'and' gate with low-level signals and finally provided to the reset outputs. The author would like to point out,

${ }^{2}$ reset signals are low active 
that three described signals reset all the devices and components on the TOTFed (instead of the reset subsystem in the VME).

The board reset is considered by the Mains and the OptoRxs as an asynchronous signal and resets the entire logic. However, there is a possibility to reset the independent components of the FPGAs using the Local bus. Each Main and OptoRx has its own reset subsystem trigged by an access to a dedicated register. In contrast to the subsystem of the VME, they diversify the written value and every bit resets a different module. For the Main the address of the reset register is OxOn 120008, where " $n$ " is the number of the FPGA. Table 3.2 explains the meaning of each bit.

For the OptoRx, similarly, the reset register is accessible from 0x0n180114 address (Table 3.3). Outputs of the reset subsystems are connected to the asynchronous inputs of the components.

\begin{tabular}{c|l|l} 
Bit no. & Function & Related instances in the VHDL code \\
\hline 0 & Reset of the processing part & TTCrx receiver, OptoRx receiver, TTS \\
1 & Reset of the settings registers & Settings \\
2 & reserved &
\end{tabular}

Table 3.2. Mapping of the reset register in the Main (address: 0x0n120008).

\begin{tabular}{c|l|l} 
Bit no. & Function & Related instances in the VHDL code \\
\hline 0 & $\begin{array}{l}\text { Reset of an analogue part } \\
\text { of the GX FPGA receivers } \\
\text { Reset of a digital part } \\
\text { of the GX FPGA receivers }\end{array}$ & s2gxBasic \\
2 & $\begin{array}{l}\text { Reset of the processing part } \\
\text { Reset of the settings registers }\end{array}$ & $\begin{array}{l}\text { InputCh, processing, TTCrx_4bit } \\
\text { Settings } \\
\text { processing }\end{array}$ \\
4 & Reset S-Link
\end{tabular}

Table 3.3. Mapping of the reset register in the OptoRx (address: 0x0n180114). 


\section{The VME}

\subsection{General overview}

The VME uses one of the smallest FPGA of Altera's Cyclone Family: EP1C4F400C8. The device is equipped with 4000 logic cells, $77 \mathrm{kbits}$ of an internal memory and 2 PLLs. It is developed at a 130-nm process node and is supplied from a $1.5 \mathrm{~V}$ voltage source. Specific information can be found in the data sheet provided by the producer [18]. In Figure 4.1 is presented a scheme of a logic element [18]. It is a well-known architecture, with a four-input LUT, which is sufficient for logic functions consisting of a few variables.

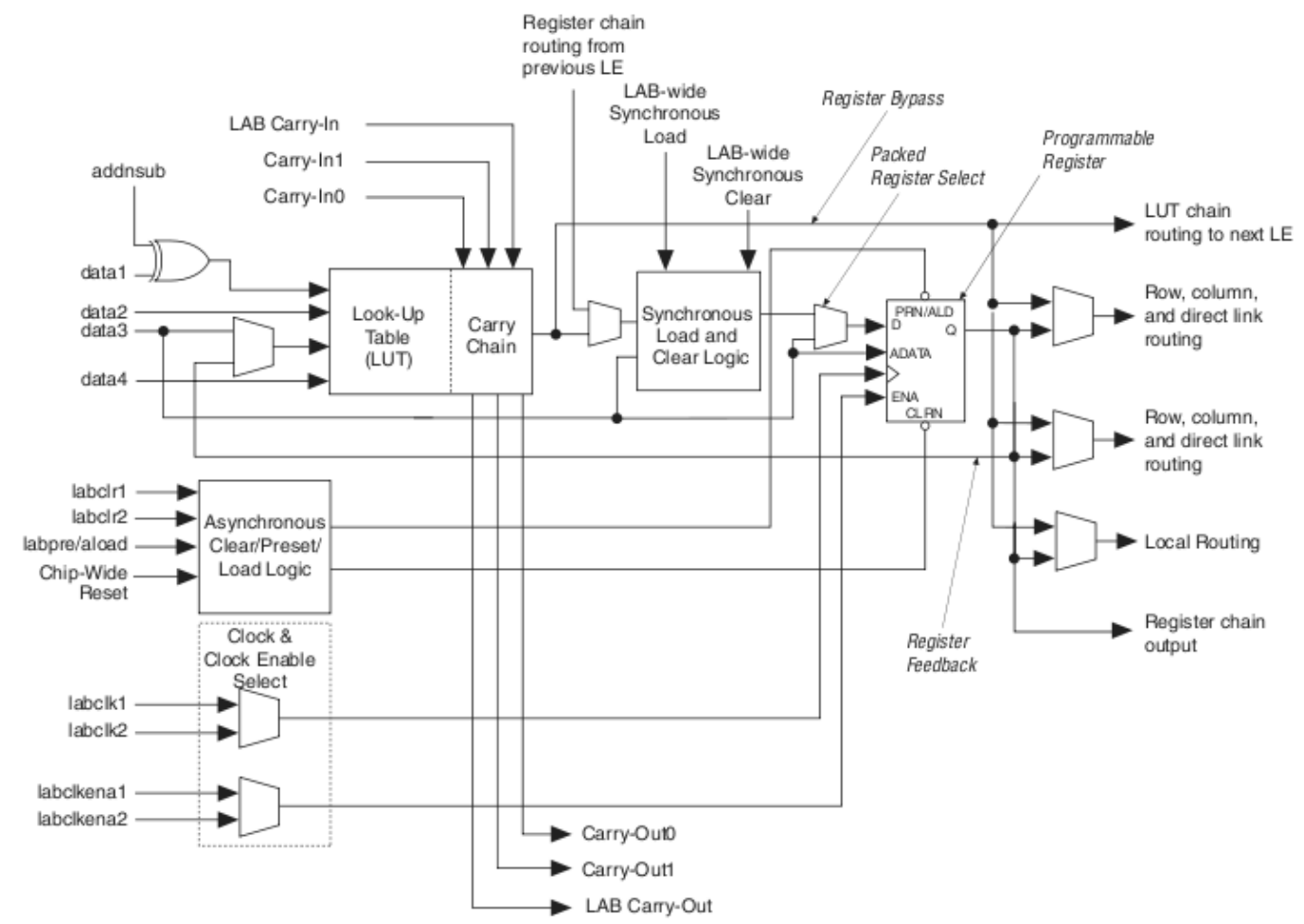

Figure 4.1. Logic Element of the Cyclone Family.

The last digit of a device number indicates a speed grade. For simple PLDs it was the time of a signal propagation through a logic cell expressed in ns. However, devices have become more complex and nowadays it can be considered only as a speed parameter, which distinguish devices within a one family. A smaller value means a faster chip. For the VME, 8 indicates the slowest option. Nevertheless, the Cyclone features were enough to satisfy not trimmed objectives: 
- acting as a bridge between VME 64 bus and the TOTFed;

- being a controller for simple interfaces like I2C, OneWire or JTag;

- implementing TTS state machine;

- supporting of the TTCrx chip.

The architecture presented in Figure 4.2 meets enumerated demands. Of course, the major block is a state machine designed accordingly to the VME bus specification [16]. It translates VME data into internal buses ${ }^{1}{ }^{1}$ and vice versa. What is more, the FSM split the device: on the left of it, each component is synchronized to the clock of $80 \mathrm{MHz}$ provided by the PLL, on the right, signals are not synchronized because the TOTEM uses an asynchronous kind of the VME bus. The most utilized is the path between the Local and the VME bus due to the data acquisition. However, from time to time, there is a need to use the simple interfaces connected to the Internal bus.

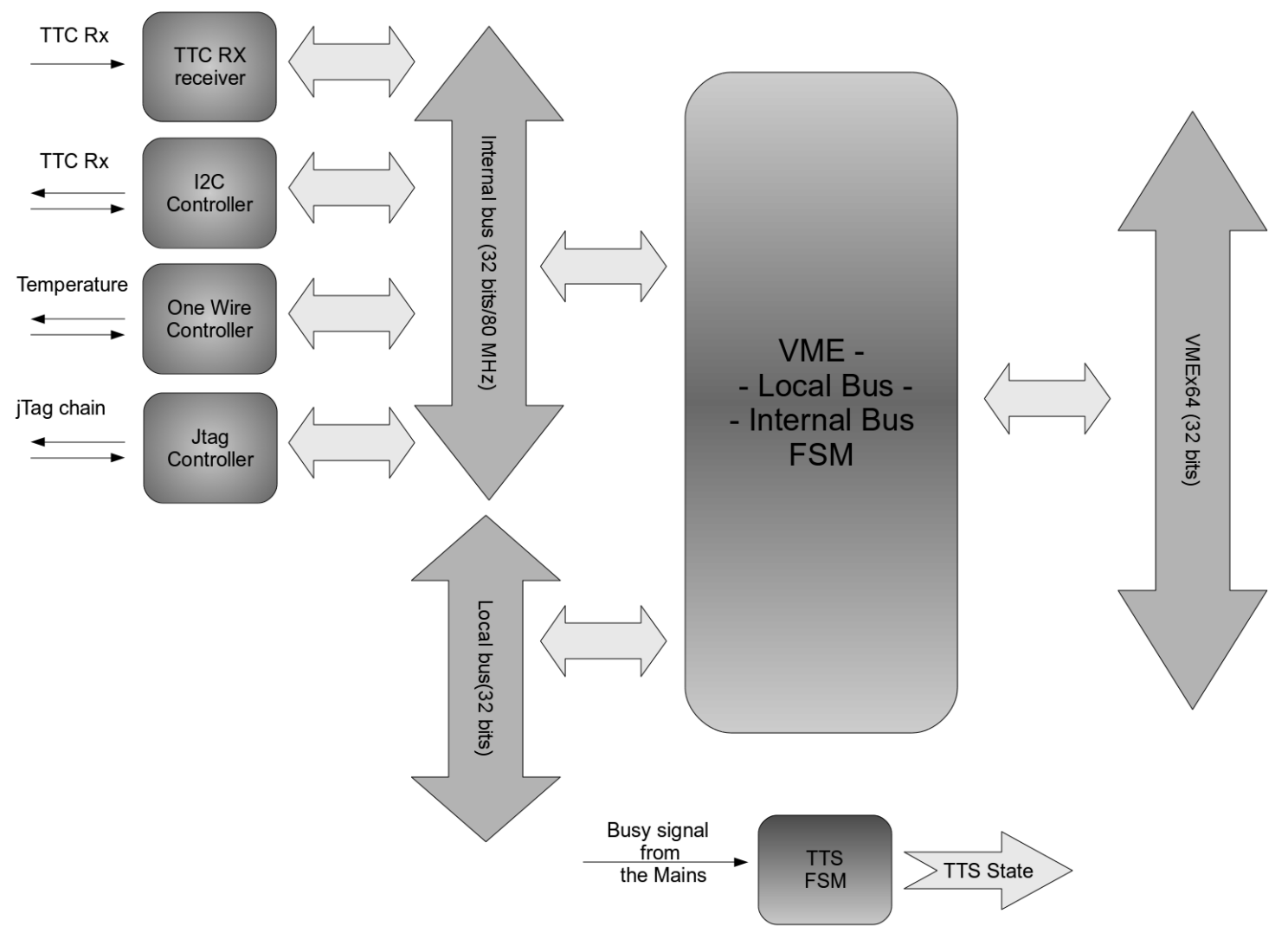

Figure 4.2. The VME architecture.

The TTCrx Receiver is used to decode the event counter (EC) and bunch counter (BC) basing on a 4-bit line controlled by the TTCrx chip. The I2C Controller allows access to the TTCrx registers. The One Wire Controller is utilized to obtain informations from embedded temperature sensors. The JTag controller gives the opportunity to boundary-scan of the TOTFed. It is a very handy feature, because allows program a FPGA remotely. At the bottom of Picture 4.2 is presented the TTS

\footnotetext{
${ }^{1}$ the VME internal bus and the Local bus
} 
machine, which based on busy signals from the OptoRxs, generates the busy state to the TTS system (chapter 2.3.4).

\subsection{Implementation}

\subsubsection{The TTCrx Controller by I2C bus}

The TOTFed has access to the TTC interface (chapter 2.3.3) through the TTCrx. Control of the chip is possible by a I2C bus. It uses the Fast-Mode Plus ( $\mathrm{Fm}+)$ mode with 7-bits addressing [19], which enables communication with speed of $1 \mathrm{Mb} / \mathrm{s}$. The entity responsible for support of the protocol in the VME is the TTCrx_I2C_Controller. The declaration of the component is presented on the listening below:

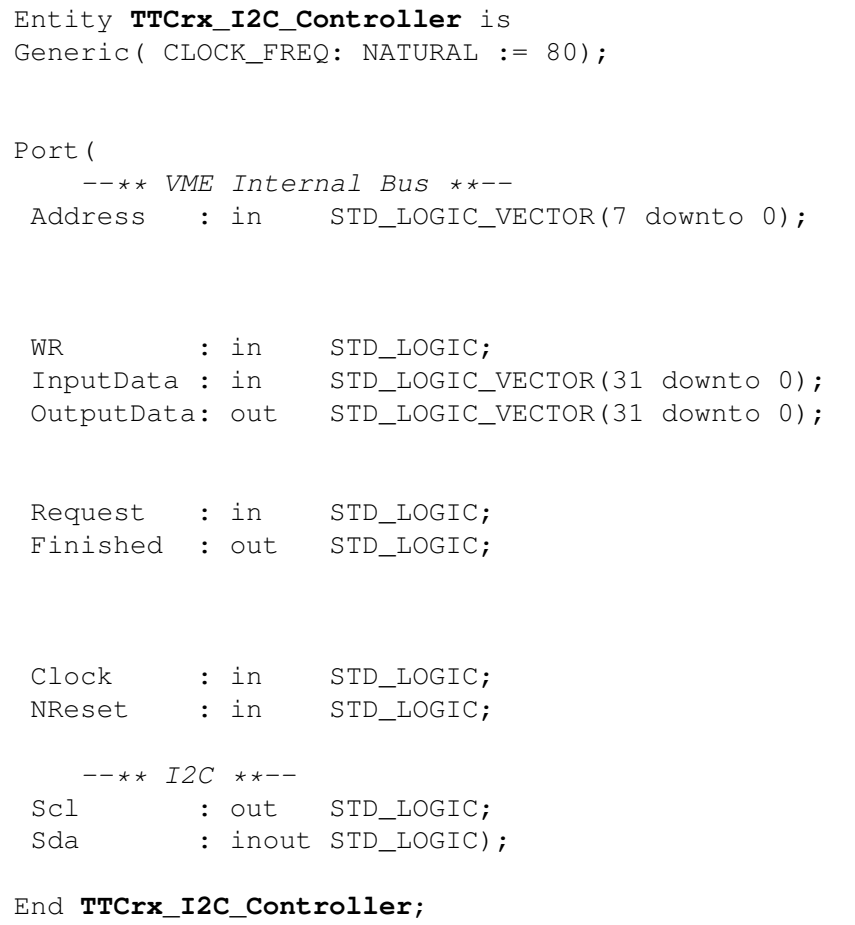

End TTCrx_I2C_Controller;

The constant CLOCK_FREQ is used by the I2C Clock Generator as a scaler (Figure 4.3) to produce $1 \mathrm{MHz}$ clock. Port Address selects the destination: if the most significant bit is one, the Address indicates internal registers of the component. In other cases the Address corresponds to the registers of the TTCrx. Detailed address mapping is presented in Table 4.3. Port WR specifies transfer direction (readout is indicated by logic one).

\begin{tabular}{c|l} 
Bit no. & Description \\
\hline 0 & $\begin{array}{l}1 \text { if the controller has not received acknowledge signal from the TTCrx. } \\
\text { It can happen if Base address is incorrect. } \\
1\end{array}$ \\
$\begin{array}{l}1 \text { if the I2C interface is busy } \\
\text { Controller can not master the interface, because SDA line is driven to low }\end{array}$
\end{tabular}

Table 4.1. Bits description in Status Register (0x81) of the TTCrx_I2C_Controller. 
Ports Input and OutputData are used to exchange information with the Internal bus of the VME (Figure 4.2). As the I2C is a 8-bit bus ${ }^{2}$ only the least significant byte is used. Rest of the bits are filled with logic zeros. However, situation changes, when user reads registers of the TTCrx. The most significant byte of the OutputData corresponds then to the status register (Table 4.1). The Request port triggers the component. It should be active during the entire work time of the controller. The Finished pin indicates the end of an operation, whether it has done successfully or failed. It stays low, until the Request is deny. The Clock by default should be connected to the source of $80 \mathrm{MHz} 3$. Pins Scl and Sda are associated to the I2C interface and can be considered, respectively, as a clock and a data line. The TTCrX_I2C_Controller is the only master on the bus, so Scl port is unidirectional.

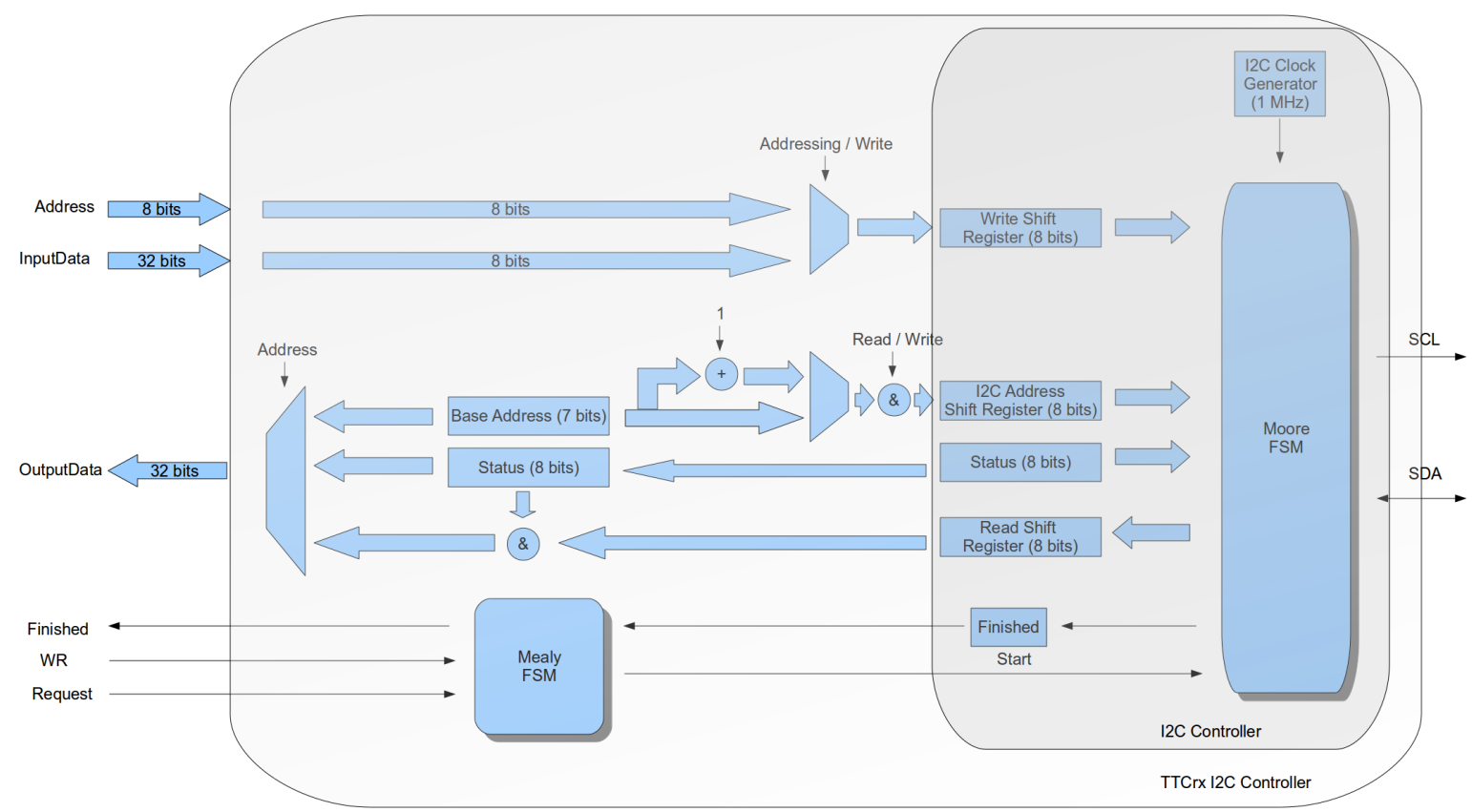

Figure 4.3. Block structure of the TTCrx I2C Controller

Figure 4.3 presents the block diagram of the TTCrx_I2C_Controller. The TTCrx occupies two addresses of the I2C bus [17|: "I2C Data Pointer" and "I2C Data". The 7-bit I2C Base Address register of the TTCrx_I2C_Controller corresponds to first of them. The TTCrx stores its own I2C address in the ID_I2C $<5: 0>$ register, whose value is set during reset of the chip 4 . The description, how to obtain the two mentioned $\mathrm{I} 2 \mathrm{C}$ addresses is presented in Table 4.2 .

The I2C_pointer is a five-bits register in the TTCrx, which points the internal register of the chip. Each access to the TTCrx via I2C has two steps

- write the register number to the $12 C_{-}$pointer;

- read or write its value from the $12 C \_$data.

\footnotetext{
${ }^{2}$ During a single access 8 bit of data are transferred

3 If not, CLOCK_FREQ has to be changed.

4 The TTCrx over the reset state changes direction of the SubAddr and Data lines from outputs to inputs. The read value is used by the chip as the ID for I2C and unicast fast commands communication described in the specification of the TTC.
} 


\begin{tabular}{l|l} 
I2C register & I2C address \\
\hline I2C_pointer & ID_I $2 \mathrm{C}<5: 0>* 2$ \\
I2C_data & ID_I $2 \mathrm{C}<5: 0>* 2+1$
\end{tabular}

Table 4.2. Calculation of the I2C addresses of the TTCrx.

As SubAddr and Data line are connected to the Mains, the ID_I2C depends on them. In consequence, the VME needs the base address of the TTCrx from software part of DAQ. The appropriate value should be written to the Base Address register (Table 4.3).

\begin{tabular}{|c|c|c|}
\hline Address & Register name & Default content \\
\hline \multicolumn{3}{|c|}{ TTCrx Internal register ${ }^{5}$} \\
\hline 0x00 & Fine Delay 1 & "00000000" \\
\hline $0 \times 01$ & Fine Delay 2 & "00000000" \\
\hline 0x02 & Coarse Delay & "00000000" \\
\hline 0x03 & Control & "10010011" \\
\hline 0x08 & Single error count $<7: 0>$ & "00000000" \\
\hline 0x09 & Single error count $<15: 8>$ & "00000000" \\
\hline OxOA & Double error count $<7: 0>$ & "00000000" \\
\hline $\mathrm{OxOB}$ & SEU error count $<15: 8\rangle$ & "00000000" \\
\hline $0 \times 10$ & $\mathrm{ID}<7: 0>$ & "00000000" \\
\hline $0 \times 11$ & MasterModeA $<1: 0>$, ID $<13: 8>$ & "00000000" \\
\hline $0 \times 12$ & MasterModeB $<1: 0>$, I2C_ID $<5: 0>$ & "00000000" \\
\hline $0 \times 13$ & Config 1 & "00011010" \\
\hline $0 \times 14$ & Config 2 & "10000100" \\
\hline $0 \times 15$ & Config 3 & "10100111" \\
\hline $0 \times 16$ & TTcrx Status & "11100000" \\
\hline $0 \times 18$ & Bunch Counter $<7: 0>$ & "00000000" \\
\hline $0 \times 19$ & Bunch Counter $<15: 8>$ & "00000000" \\
\hline $0 \times 1 \mathrm{~A}$ & Event Counter $<7: 0>$ & "00000000" \\
\hline $0 x 1 B$ & Event Counter $<15: 8>$ & "00000000" \\
\hline $0 \times 1 C$ & Event Counter $<23: 16>$ & "00000000" \\
\hline \multicolumn{3}{|c|}{ TTCrx I2C Controller internal registers: } \\
\hline $0 \times 80$ & Base Address & 0x00000000 \\
\hline $0 \times 81$ & TTcrx Controller Status & 0x00000000 \\
\hline
\end{tabular}

Table 4.3. TTCrx I2C Contoller Address mapping.

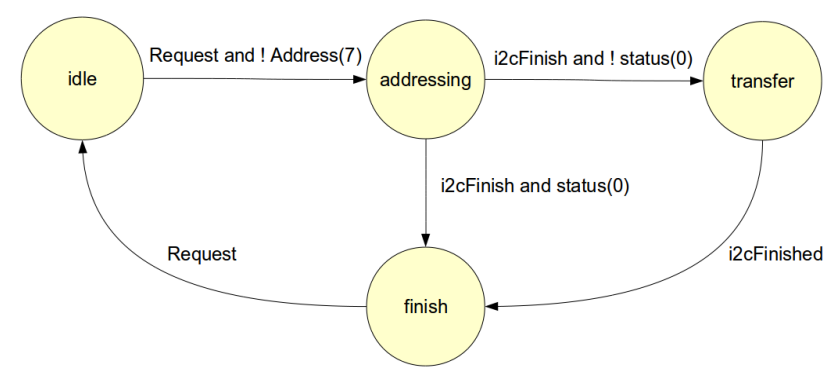

Figure 4.4. States diagram of the Mealy's FSM in the TTCrx_I2C_Controller.

\footnotetext{
5 The description of the registers can by find in the manual of the TTCrx 17
} 
The FSM in the TTCrx_I2C_Controller (Figure 4.3) toggles mainly between 2 states: addressing and transfer. The Mealy's approach in the state machine results the fast implementation. The states diagram is presented in Picture 4.4. It simply switch multiplexer that provides data to low-level entity in the design (I2C_Controller) and asserts Finish flag when operation has done (state: Finish).

\section{I2C_Controller}

The I2C_Controller is a low-level component, which accomplishes the I2C transitions. Underneath is presented the declaration of the entity:

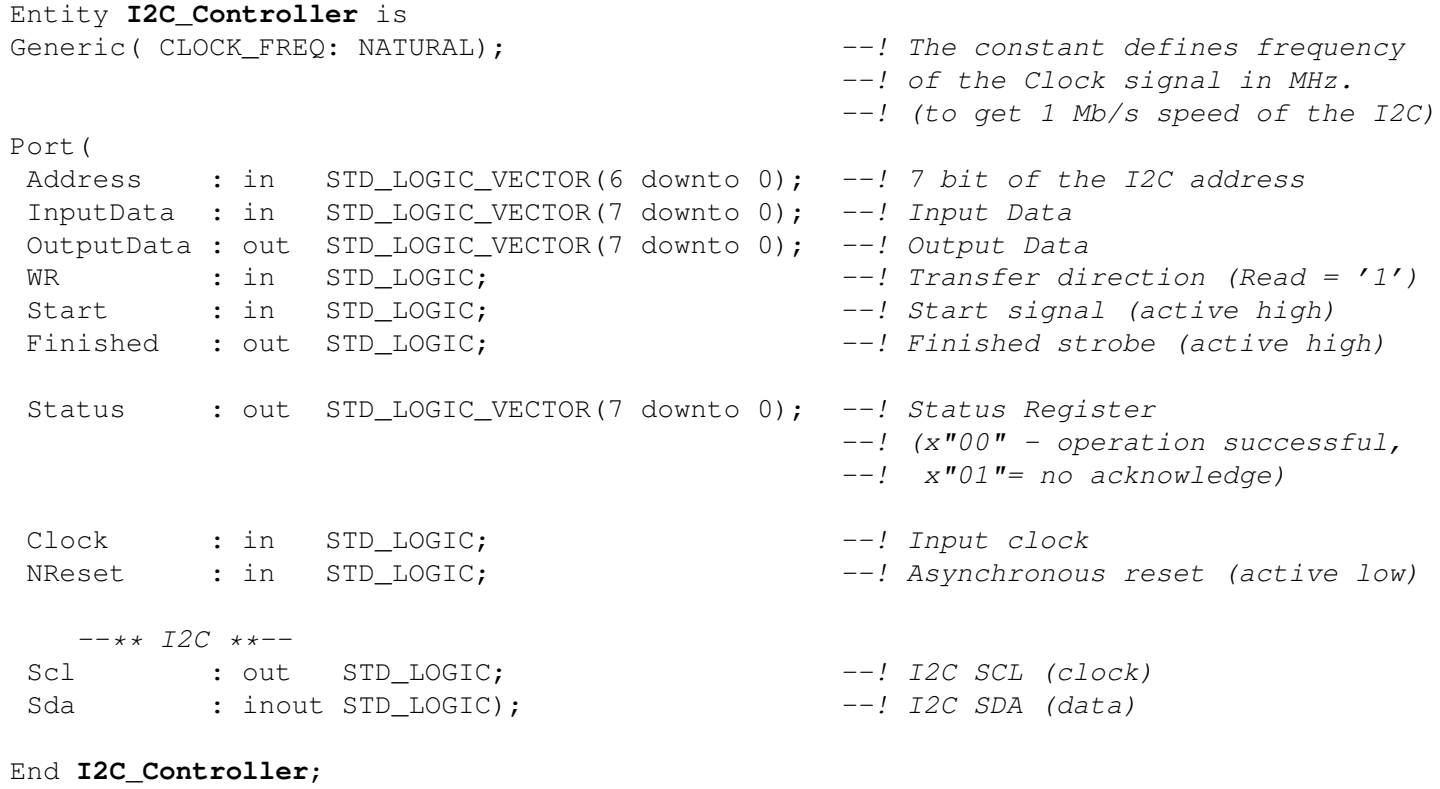

The ports destination of the I2C_Controller is alike to the TTCrx_I2C_Controller. As it is presented in Figure 4.3, the component mainly consists of a FSM. The Moore approach combined with a one-hot state codding lets to obtain stable transitions on the external pins (SCL and SDA) of the VME FPGA. Consequently, the FSM has a quite lot of states, whose diagram is presented in Figure 4.5. The states with the suffix "_s" from stable, indicates states, where the SCL is high and data bit on the $S C L$ has to be valid. All transactions on $S D A$ line 6 are done a one Clock cycle after a falling edge of the SCL. The FSM uses a counter to count number of consecutive bits, which are proceeded by the shift registers. The Finish flag informs the high-level component, if a transfer was completed. It is asserted only a one Clock cycle, which is enough for the external Mealy's state machine.

\begin{tabular}{c|c} 
Status code & Description \\
\hline Ox00 & Operation succeed \\
Ox01 & No acknowledge signal from slave \\
Ox02 & Slave is Busy (SDA driven to GND)
\end{tabular}

Table 4.4. Description of the status register values.

\footnotetext{
${ }^{6}$ instead of Start and Stop conditions
} 
The I2C Clock Generation generates the signal of $1 \mathrm{MHz}$, which is used by the FSM to drive the interface with an appropriate timing. To reduce power consumption, related to a transistors switching, in the idle state, the clock generator is disabled.

The status registers informs about results of an last operation. A meaning of specific values are gathered in Table 4.4

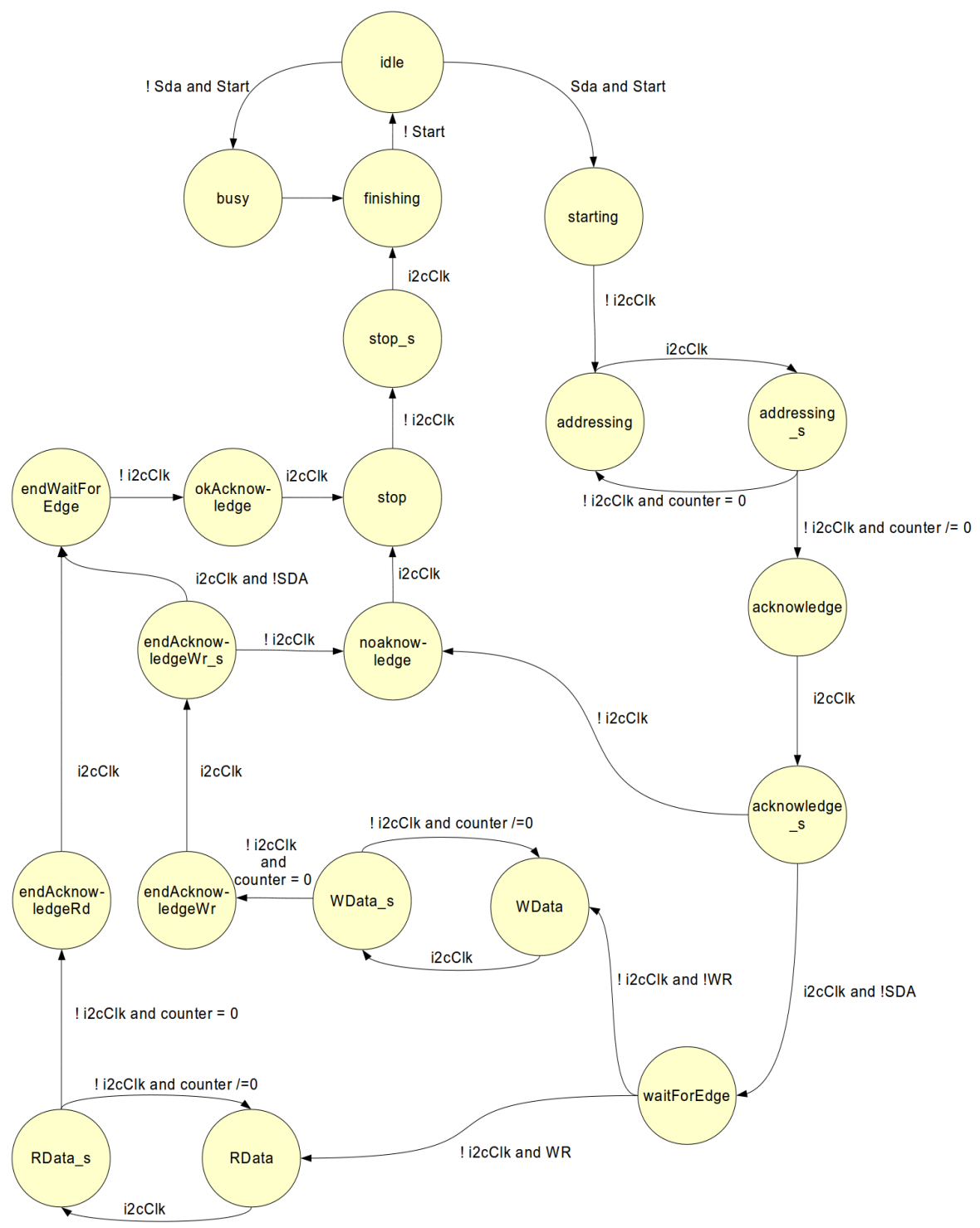

Figure 4.5. States diagram of the Moore's FSM in the I2C_Controller. 


\section{Example usage}

The waveform in Figure 4.6 presents example write access to the Coarse Delay register. The transfer begins from the start state of the I2C bus (7 $085 \mathrm{~ns}$ ): the falling edge of the SDA during a high level of the SCL. The controller addresses the slave and waits for an acknowledge from it - the low level of the SDA during high SCL (15 $915 \mathrm{~ns})$. Next, the TTCrx_I2C_Controller writes the appropriate address to the I2C_pointer register of the TTCrx (state: addressing) and again checks the acknowledge. Afterwards, the I2C stop is sent - the rising edge of the SDA accompanying a high level of the SCL (27 $090 \mathrm{~ns})$. After this, the TTCrx_I2C_Controller goes to the transfer state, which is commenced again with the I2C start state (27 $625 \mathrm{~ns}$ ). Then, sequentially, the I2C_data register is addressed, the acknowledge is checked (36 $445 \mathrm{~ns}$ ), the appropriate data are sent, the end acknowledge is affirmative and master finishes the transmission with the I2C stop (47 $615 \mathrm{~ns}$ ). At the end, the component asserts the Finished flag and waits for release of the Request. During the readout of the I2_data register, the last acknowledge should be asserted by the controller. However, as it is a last byte of a single transfer, it is specific situation, described in the specification [19], and I2C acknowledge state does not occur7?

\footnotetext{
7 This fact explains why after the EndAcknowledgeRd state from the diagram 4.5 is only one path directly to the EndWaitForEdge state regardless of the SDA
} 


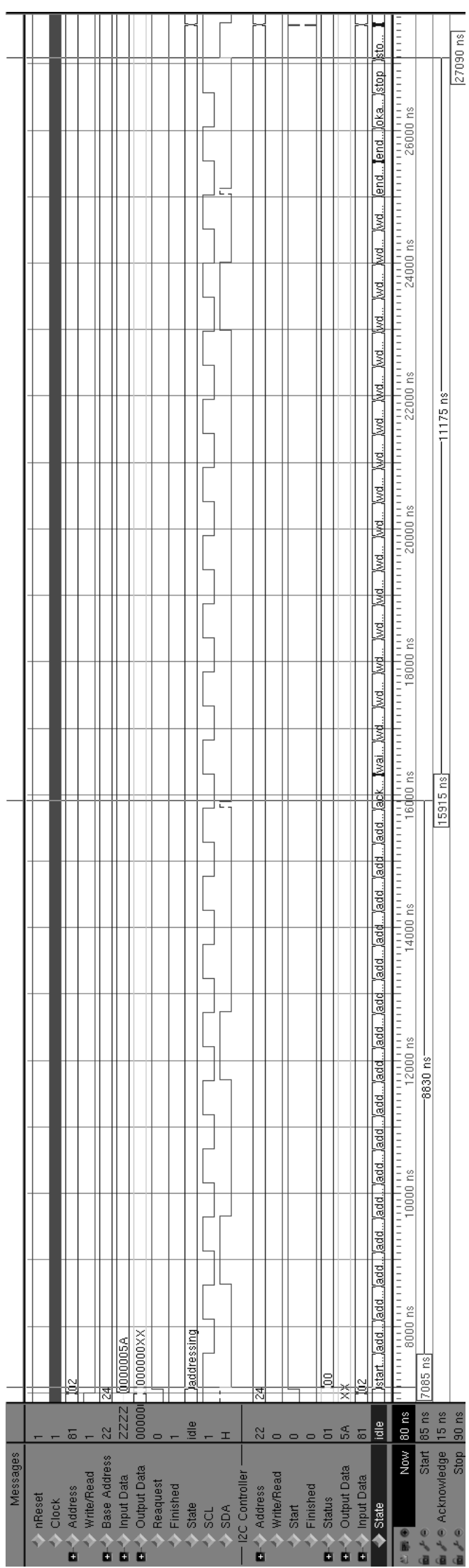

(a) Addressing

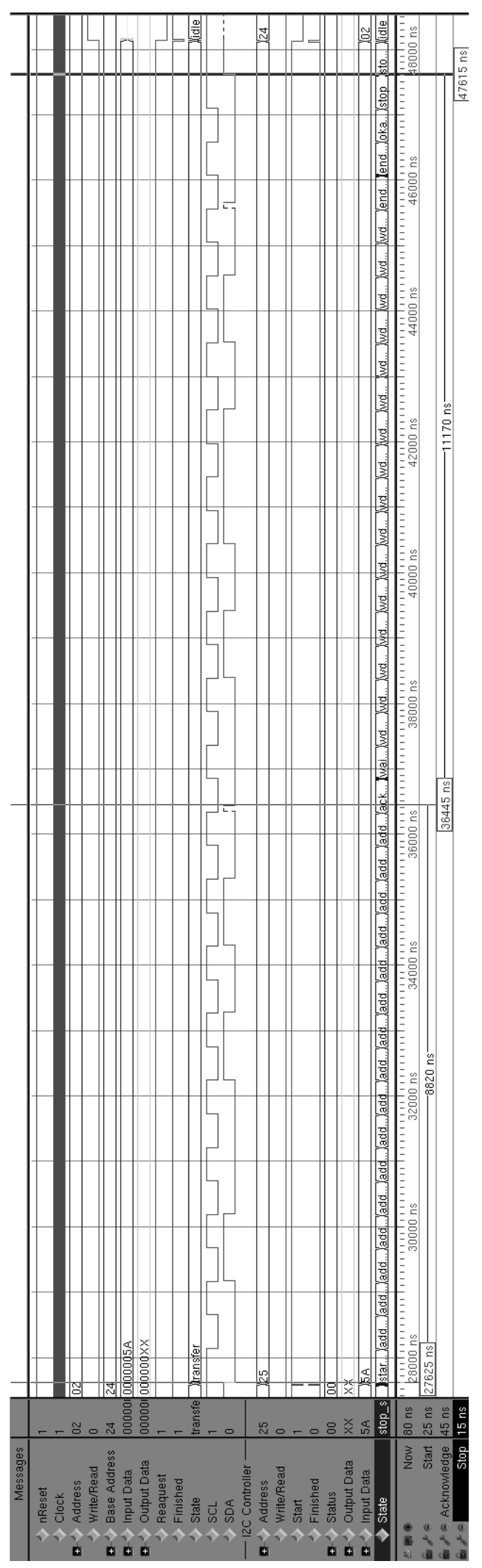

(b) Transfer

Figure 4.6. Waveform of write access to the Coarse Delay register of the TTCrx (Base address: 0x22). 


\subsubsection{VME Reset Subsystem}

The VME Reset Subsystem is responsible by the reset policy, which was roughly described in Section 3.4. The following listening presents a entity declaration:

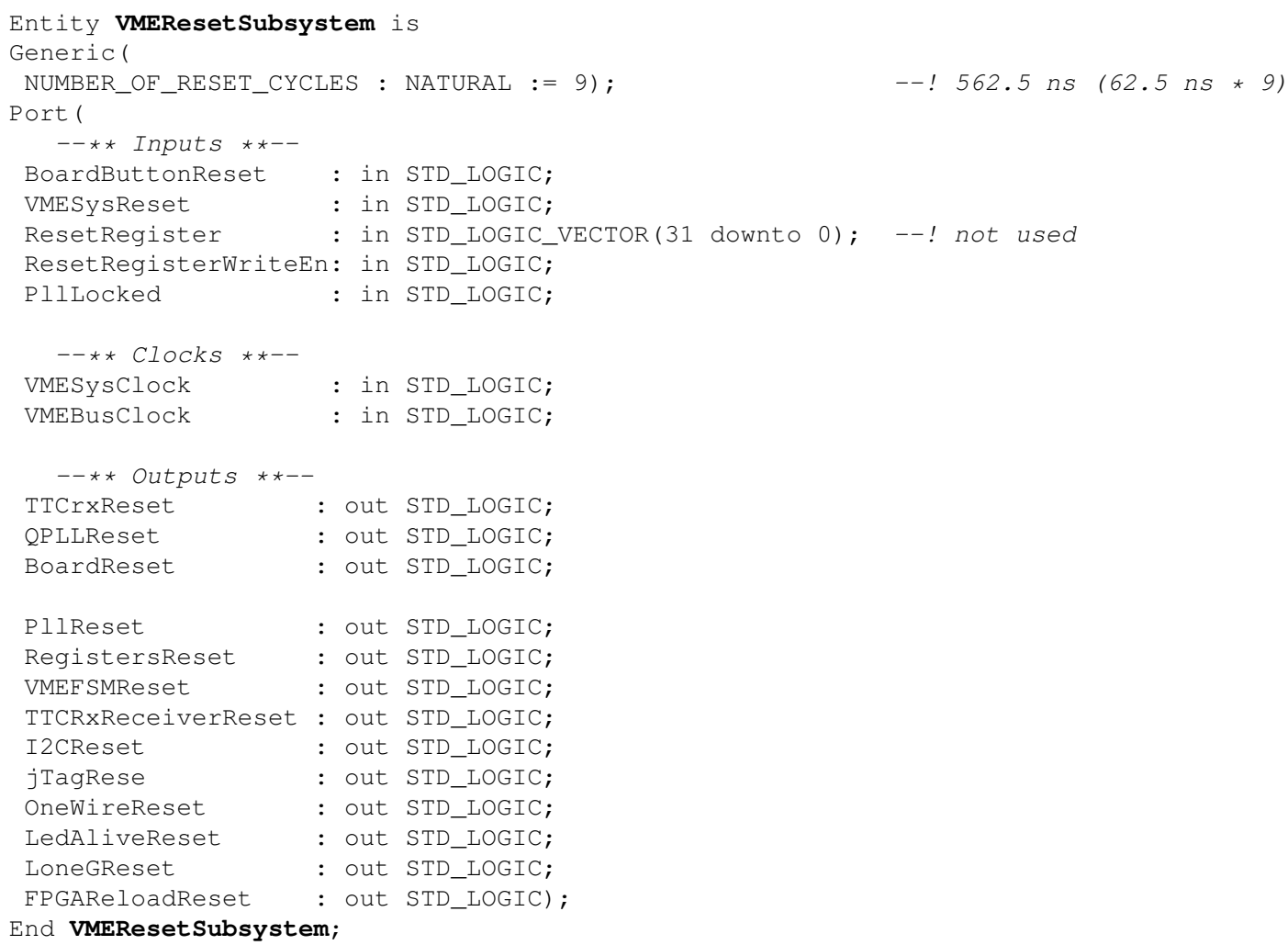

The generic value NUMBER_OF_RESET_CYCLES specifies a width of the reset pulse appearing at each of the outputs, caused by a synchronous write access to the MODULE_RESET register. The diagram 4.7 shows structure of the block.

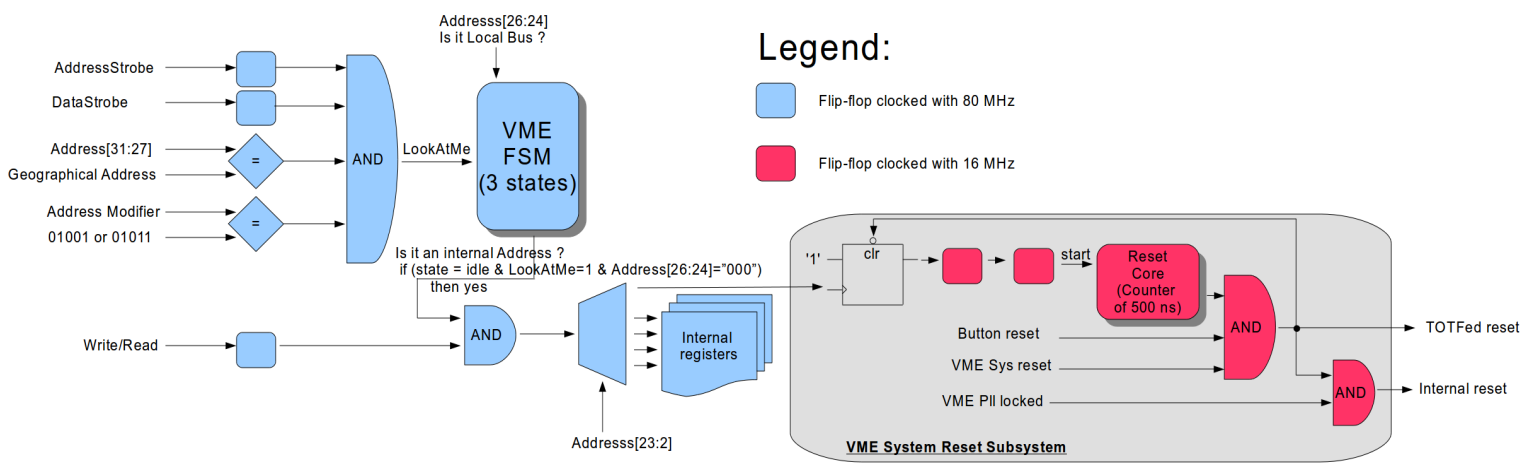

Figure 4.7. Structure of the VME reset subsystem against the background of the rest related logic.

As during the reset condition, QPLL is reset as well, the component uses the VMESysClock of $16 \mathrm{MHz}$ provided by the VME bus to count width of the generated pulse during the synchronous reset. This forces to use a clock domain bridge in form of a chain of flip-flops. The first one, is the flip-flop, which reacts on a rising 
edge of the ResetRegisterWriteEn. As it is only a one latch, it does not consume a global clock line 8 , but uses only a local LAB clock multiplexer.

The next, two, red flip-flops synchronize the ResetRegisterWriteEn to the VMESysClock domain. Figure 4.7 presents the external logic related to the module. It was critical, to avoid feedback of logic, which could cause metastable transitions. The TOTFed reset signal from the figure is associated with the TTCrxReset, QPLLReset, BoardReset and the PLLReset ports. Rest of the outputs use the Internal reset from the figure, which determines a reset condition also because of lack of a PLL synchronization. Although, that current project does not give the opportunity to distinguish an internal component to be reset, this feature can by simply provided by means of the ResetRegister which is implemented, but not used.

\section{ResetCore}

The VMEResetSubsystem uses the low-level entity called ResetCore. It is the common module used by all FPGAs on the TOTFed. The ResetCore simply asserts the ResetOutput for a NUMBER_OF_RESET_CYCLES Clock cycles, since the Start strobe is activated. Below is presented the component declaration:

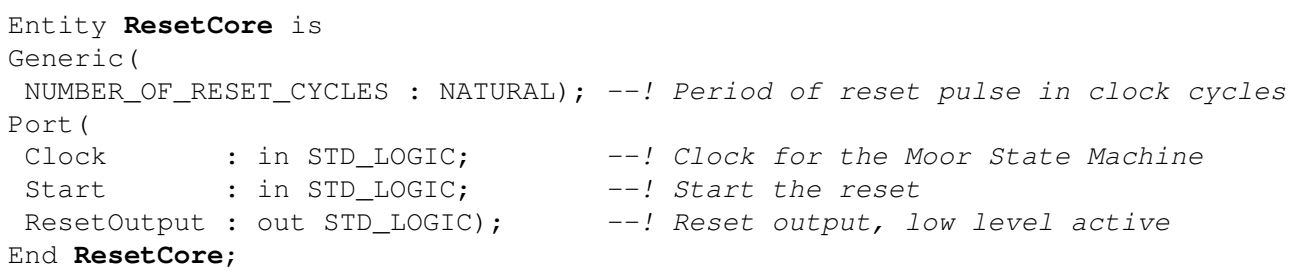

\section{Example usage}

Figure 4.8 presents work of the VME Reset Subsystem. The waveform can be divided into 3 parts. The first two illustrate behaviour of the module on respectively the Button reset ( $20 \mathrm{~ns}$ ) and the VME System reset (565 ns). The last one (1 $400 \mathrm{~ns}$ ) shows the synchronous reset. The start signal (Reset register write enable) spreads through latch and the two flip-flop changing clock domain. Finally the Reset core generates reset pulse (1 $594 \mathrm{~ns}$ ) of width $562.5 \mathrm{~ns}$. The reset signal for the internal components lasts longer due to the time needed by the PLL to lock a clock.

${ }^{8}$ the Cyclone has 8 of them 


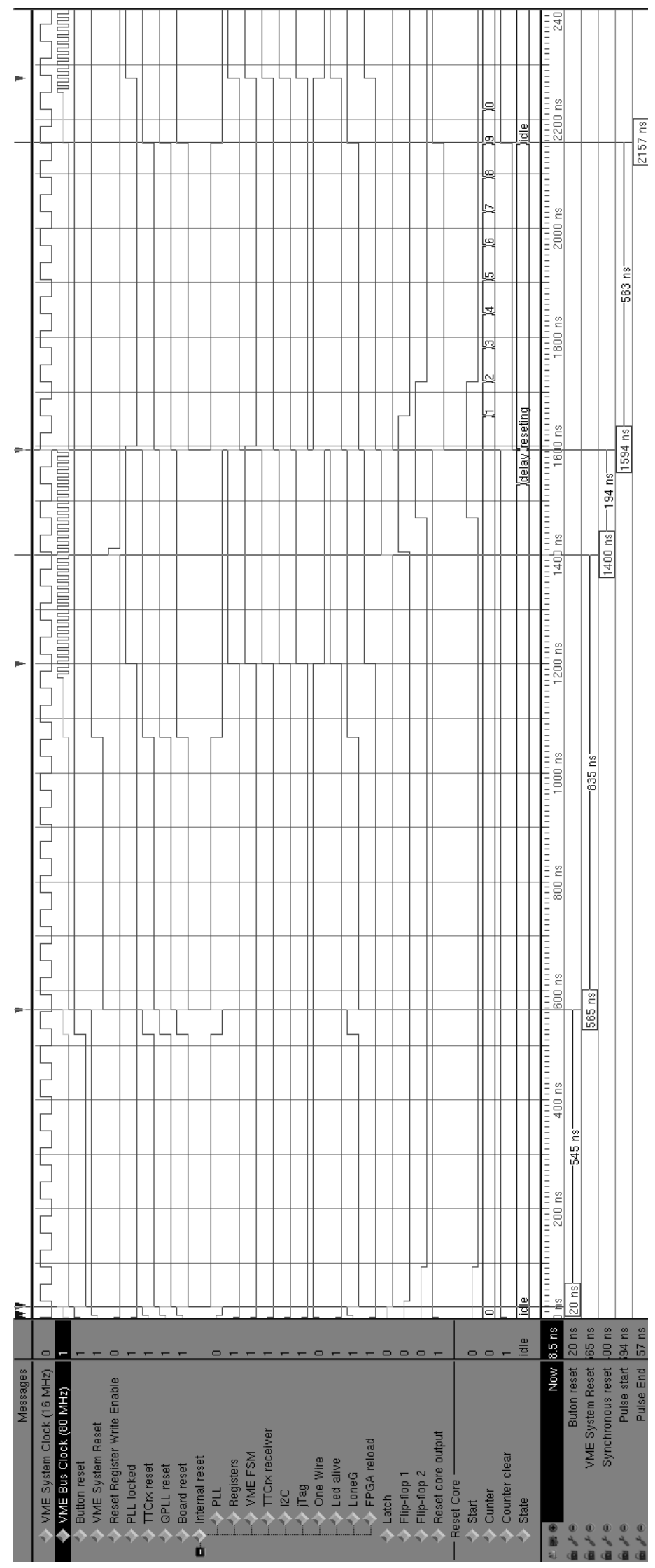

Figure 4.8. Example usage of the VME Reset Subystem. 


\subsubsection{VME Controller}

The VME Controller is responsible for multiplexing data from several data sources: the VME bus, the Local bus and the internal components, which give access to further interfaces like the I2C, the One Wire or the jTag. The implementation should ensure an easy way to expand the system to new entities. At the same time, it has to be reliable, because experiment data are transferred through this module, and quick enough to allow working with assumed $1 \mathrm{kHz}$ trigger rate. The component declaration is presented below:

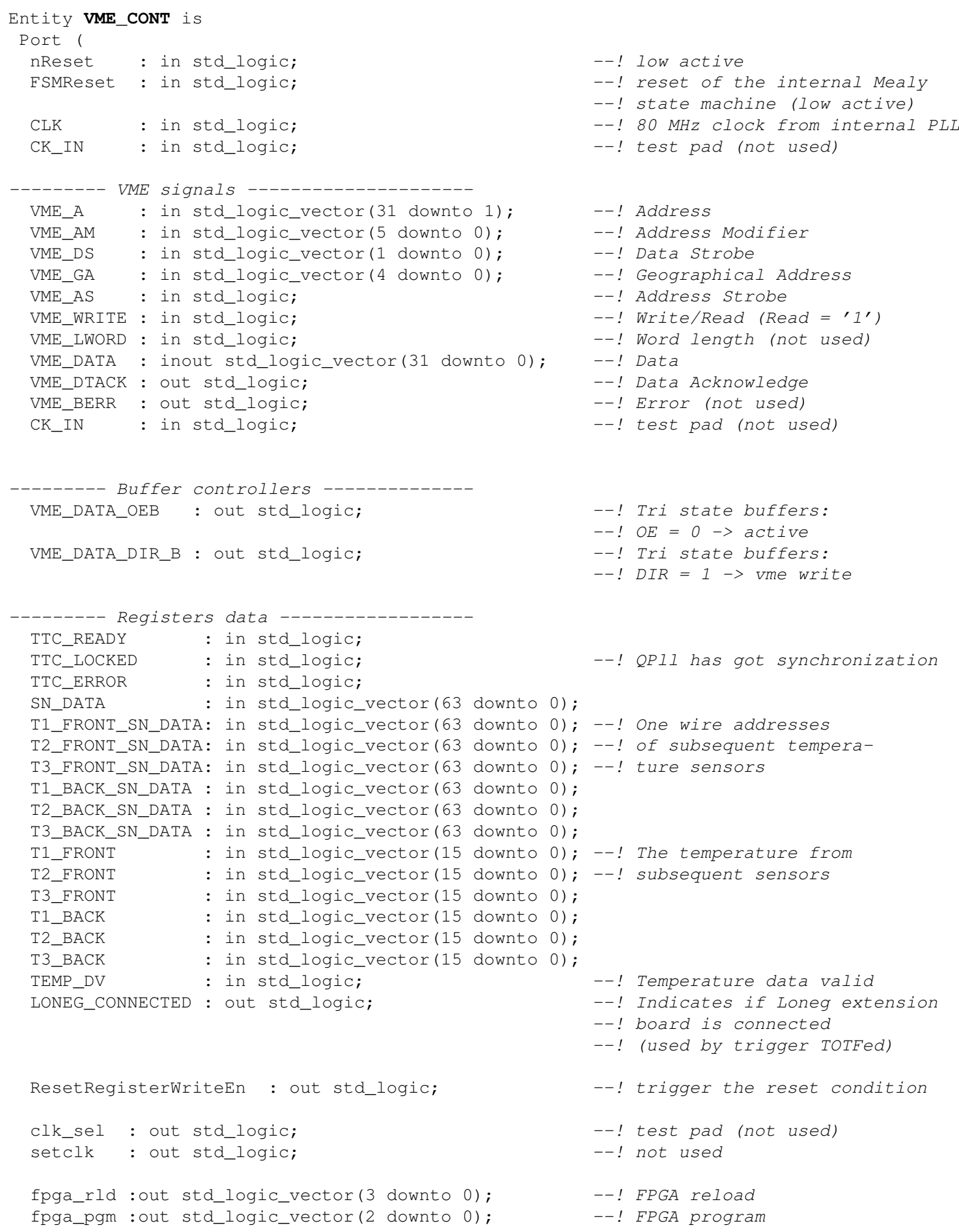




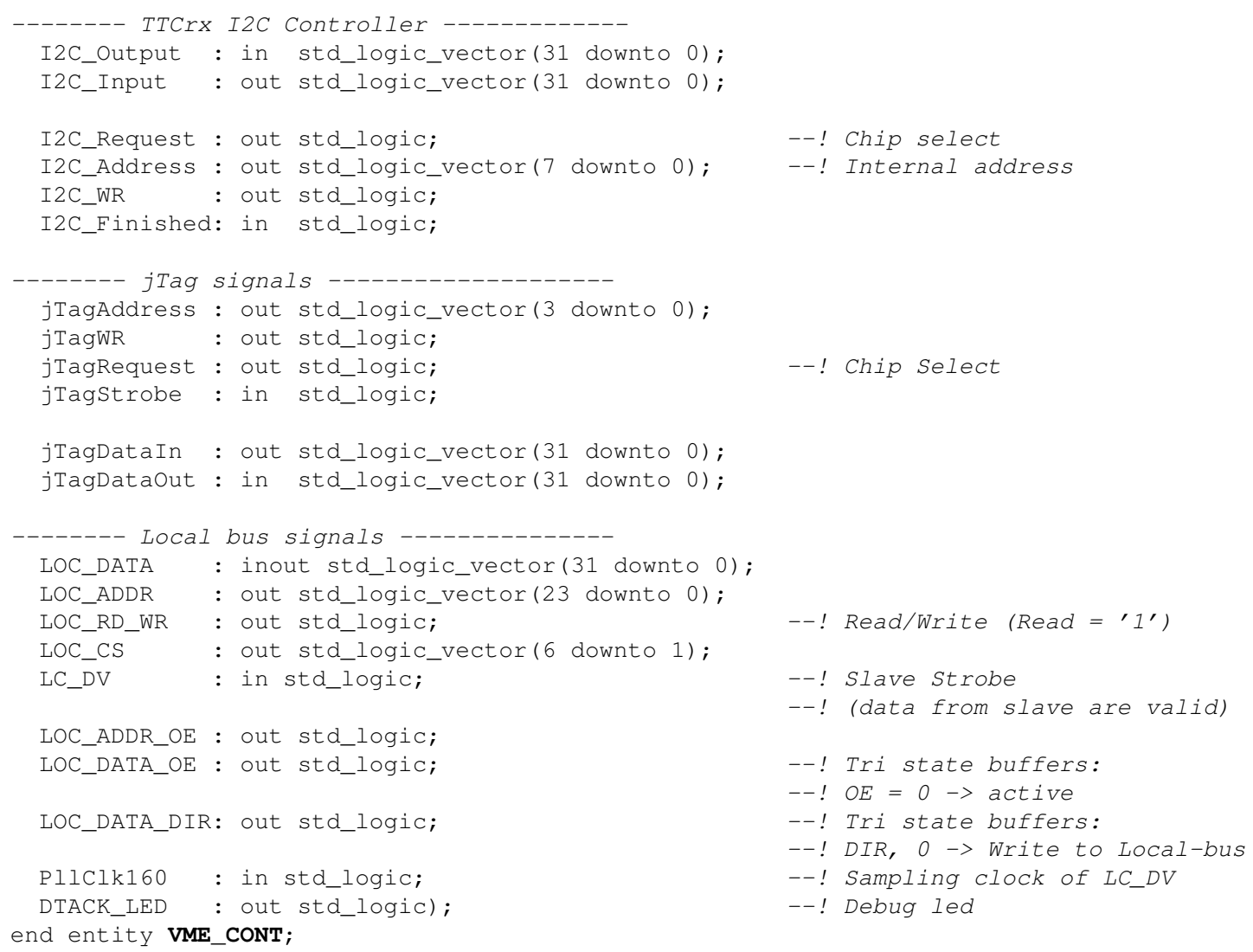

One can notice, that not all pins are used. This is because entity needs an upgrade. For example, VME_CONT should fully support the VME bus, the structure of the module should be hierarchical, etc. The current versions was validated in real conditions, before the long start of the LHC in March 2010 and proved, that works correctly. As the entity is critical for DAQ, any changes require a deeply debugging, which cannot be done during the LHC work. This fact has driven to postpone any changes to the temporary suspend time of LHC in 2012.

The VME_CONT is presented in Figure 4.9. The heart of the component is a small FSM. The Mealy's approach results with the quick reaction time on incoming signals and in consequence, increases performance. However, it is sensitive on any incoming glitches and creates peaks at the outputs. From this reason, input registers have been used. What is more, the critical signal from a speed point of view, the $L C \_D V \sqrt{9}$, is sampled with a faster $160 \mathrm{MHz}$ clock. The transfer procedure starts when the LookAtMe condition is satisfied. Right now, the only supported VME modes, selected by address modifier, are the 32-bit single and block read/write. For this reason, although the VME crate supports the VME64 standard, it is not possible to obtain performance benefits issued by usage of 64 bits.

In Figure 4.9, the internal registers map ports of the rest modules in the VME FPGA, like the TTCrx I2C Controller, in a one address space. They correspond as well to setting registers, which control a work of the device. The full list of the

9 The signal indicates valid data on the Local bus during readout. It is shared between all Mains and uses pull-up resistors, which are not recommended to high-speed interfaces (the cause is explained in Section 5.2.1. For this reason performance of this part, especially in a block readout mode is critical. 
registers with the VME addresses offset is presented in Section 4.2.3. The crucial, from synthesis point of view, were multiplexers surrounding the internal registers, so their select and input ports use registers to cut a critical paths and satisfy timing requirements. As the Local and VME bus is bidirectional, following objective for the FSM is to manage the output tri-state buffers.

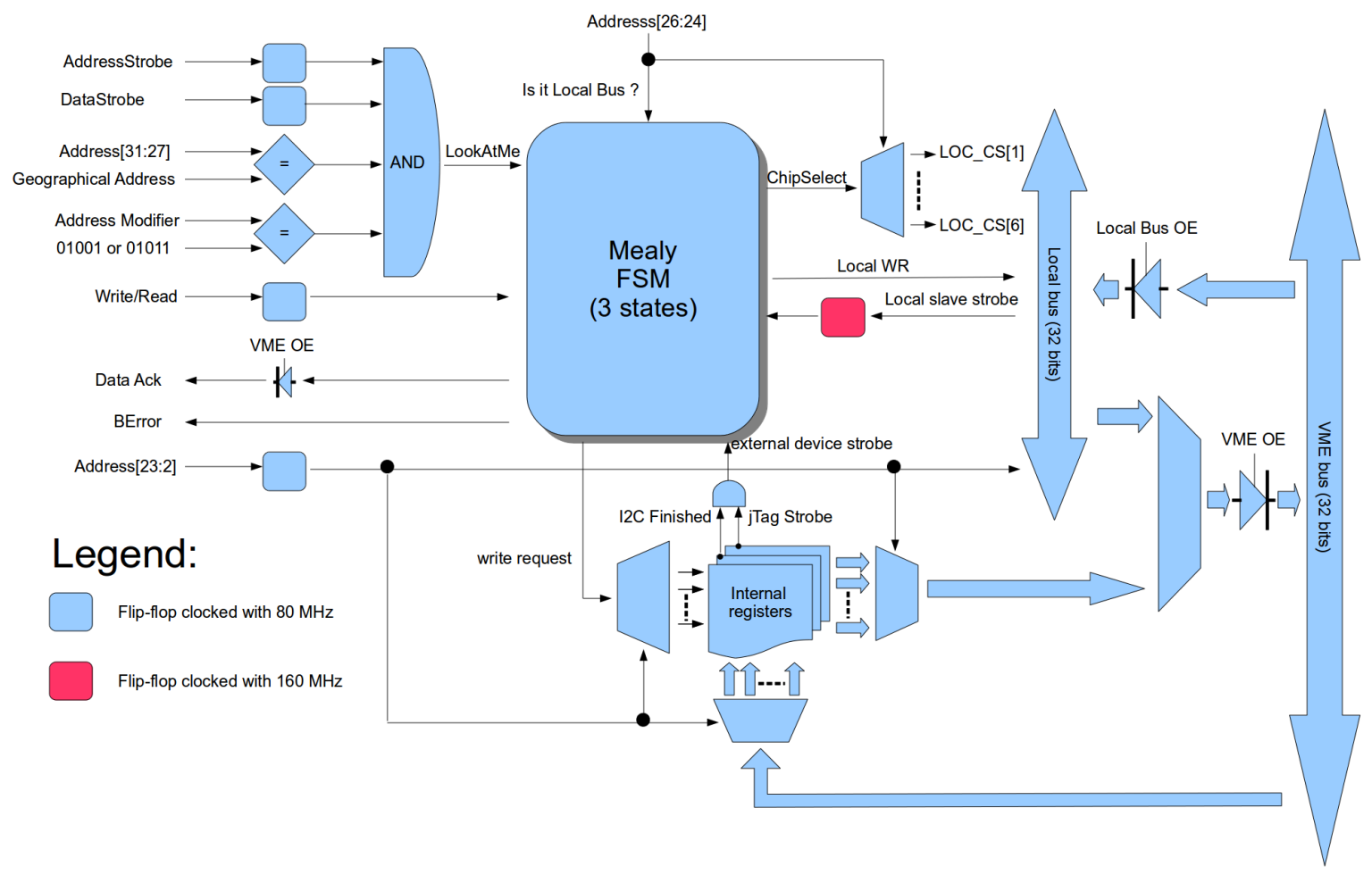

Figure 4.9. The VME Controller entity.

\section{VME Controller state machine}

The state transitions are presented in Figure 4.10. It has to be universal because of the multiple data recipient. In the SO state, the FSM waits until the LookAtMe condition is not satisfied. When it happen, before the FSM has managed to go to the $S 1$ state, it starts driving multiplexers, which choose a destination 10 and a transfer direction. The reader should be aware, that outside of the FPGA, on the TOTFed, exist external buffers: between the VME FPGA pins and the VME, Local buses lines. They are relatively slow, especially if one compares the time of switch from high-impedance (7.5 ns) to the clock of the FSM (12.5 ns). For this reason, they do not wait for the LookAtMe but relay on a lighter condition: active address strobe, which use to be asserted 12.5-25 ns faster than data strobe by the VME driver.

In the $S 1$ state the FSM waits for the end of the transaction. If an access is made to the one of the internal registers (instead of the registers, which need an access to the external device like the TTCrx I2C Controller), it asserts Data Acknowledge signal immediately. For the external devices it expects the finish strobe from an appropriate module. In case of the Local bus access, the state machine will wait in

10 the Local bus or the internal registers 


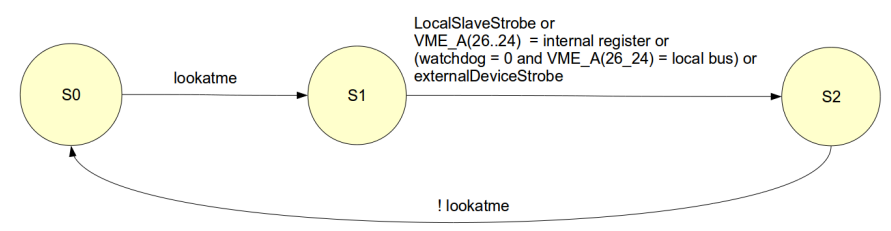

Figure 4.10. The VME Controller entity.

the $S 1$ state as long as the $L C \_D V$ stay low or an internal programmable watchdog achieve 0. As the FSM uses Mealy's implementation, the Data Acknowledge is asserted still in the $S 1$ state.

The S2 state is used to finish the transition. The state machine maintains the Data Acknowledge and simply waits for a release of all strobes by the VME driver.

\section{Address Space of the VME}

Table 4.5 presents mapping of all register of the TOTFed. The address follows a form presented in Section 3.3 . 


\begin{tabular}{|c|c|c|c|c|c|}
\hline $\begin{array}{l}\text { FP- } \\
\text { GA }\end{array}$ & Address Name & $\begin{array}{l}\text { Offset } \\
\text { (hex) }\end{array}$ & $\mathrm{R}$ & $\mathrm{W}$ & Comment \\
\hline \multirow{31}{*}{$\sum_{>}^{1}$} & MODULE_ID & 00000000 & 1 & 0 & Fixed pattern 0xFED00380 \\
\hline & BASE_ADDRESS & 00000004 & 1 & 1 & Geographical address \\
\hline & FPGA_DESIGN_RELOAD & 00000008 & 1 & 1 & not implemented \\
\hline & VME_VERSION & 0000000c & 1 & 0 & $\begin{array}{l}\text { Firmware version, } \\
\text { current: } 0 x B 06\end{array}$ \\
\hline & MODULE_RESET & 00000010 & 0 & 1 & $\begin{array}{l}\text { Any write access resets an all } \\
\text { TOTFed }\end{array}$ \\
\hline & LOCAL_READ_BACK & 00000014 & 1 & 0 & $\begin{array}{l}\text { Direct readout of data lines of } \\
\text { the Loal bus }\end{array}$ \\
\hline & VME_TEST_1 & 00000018 & 1 & 1 & Read/Write test register \\
\hline & VME_TEST_2 & $0000001 \mathrm{c}$ & 1 & 1 & Read/Write test register \\
\hline & BOARD_SERIAL_1 & 00000020 & 1 & 0 & Least(1) \\
\hline & BOARD_SERIAL_2 & 00000024 & 1 & 0 & $\begin{array}{l}\text { and most(2) significant bytes } \\
\text { of the } 64 \text { bits TOTFed serial } \\
\text { number (based on DS2401) }\end{array}$ \\
\hline & T1_FRONT_SERIAL_1 & 00000028 & 1 & 0 & Least (1) \\
\hline & T1_FRONT_SERIAL_2 & $0000002 c$ & 1 & 0 & and most (2) significant bytes \\
\hline & T2_FRONT_SERIAL_1 & 00000030 & 1 & 0 & of 64 bits serial numbers \\
\hline & T2_FRONT_SERIAL_2 & 00000034 & 1 & 0 & of three front \\
\hline & T3_FRONT_SERIAL_1 & 00000038 & 1 & 0 & thermometers (DS1822) \\
\hline & T3_FRONT_SERIAL_2 & $0000003 \mathrm{c}$ & 1 & 0 & \\
\hline & T1_BACK_SERIAL_1 & 00000040 & 1 & 0 & Least (1) \\
\hline & T1_BACK_SERIAL_2 & 00000044 & 1 & 0 & and most (2) significant bytes \\
\hline & T2_BACK_SERIAL_1 & 00000048 & 1 & 0 & of 64 bits serial numbers \\
\hline & T2_BACK_SERIAL_2 & $0000004 \mathrm{c}$ & 1 & 0 & of three back \\
\hline & T3_BACK_SERIAL_1 & 00000050 & 1 & 0 & thermometers (DS1822) \\
\hline & T3_BACK_SERIAL_2 & 00000054 & 1 & 0 & \\
\hline & TEMP_T1_FRONT & 00000080 & 1 & 0 & $\begin{array}{l}\text { Temperature result: from first } \\
\text { front thermometer }\end{array}$ \\
\hline & TEMP_T2_FRONT & 00000084 & 1 & 0 & $\begin{array}{l}\text { from second front thermome- } \\
\text { ter }\end{array}$ \\
\hline & TEMP_T3_FRONT & 00000088 & 1 & 0 & from third front thermometer \\
\hline & TEMP_T1_BACK & $0000008 \mathrm{c}$ & 1 & 0 & $\begin{array}{l}\text { Temperature result: from first } \\
\text { back thermometer }\end{array}$ \\
\hline & TEMP_T2_BACK & 00000090 & 1 & 0 & $\begin{array}{l}\text { from second back thermome- } \\
\text { ter }\end{array}$ \\
\hline & TEMP_T3_BACK & 00000094 & 1 & 0 & from third back thermometer \\
\hline & WATCHDŌG & 00000098 & 1 & 1 & $\begin{array}{l}16 \text { bits watchdog used for } \\
\text { transfers on the Local bus }\end{array}$ \\
\hline & LONEG_CONF & $0000009 c$ & 1 & 1 & $\begin{array}{l}\text { Bit } O \text { : LoneG is connected to } \\
\text { the mFEC mezzanine (set by } \\
\text { user) }\end{array}$ \\
\hline & MODULE_STAT & 000000a0 & 1 & 0 & $\begin{array}{l}\text { Bit 2: TTC Error } \\
\text { Bit 1: TTC Locked } \\
\text { Bit 0: TTC Ready }\end{array}$ \\
\hline
\end{tabular}




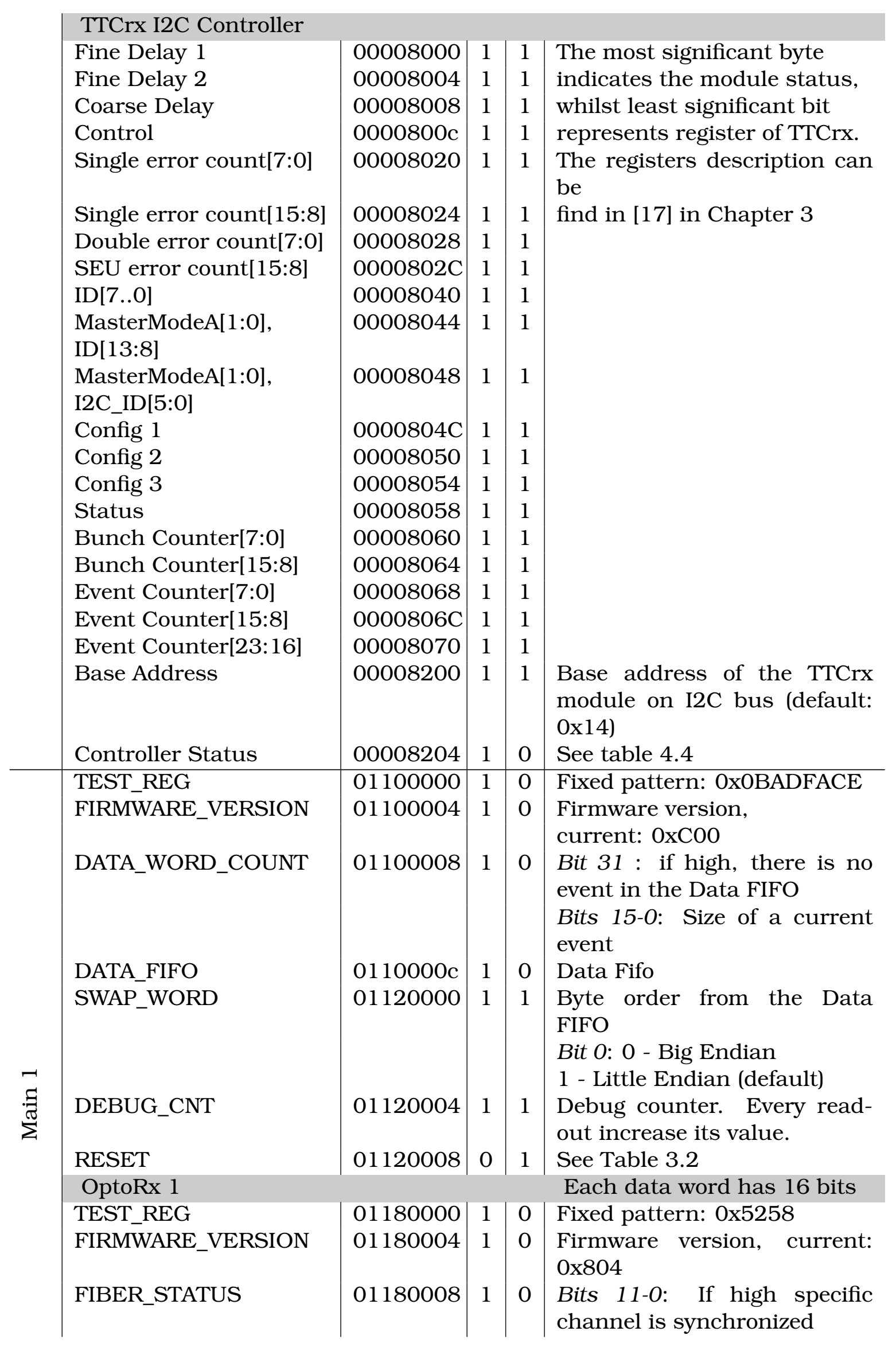




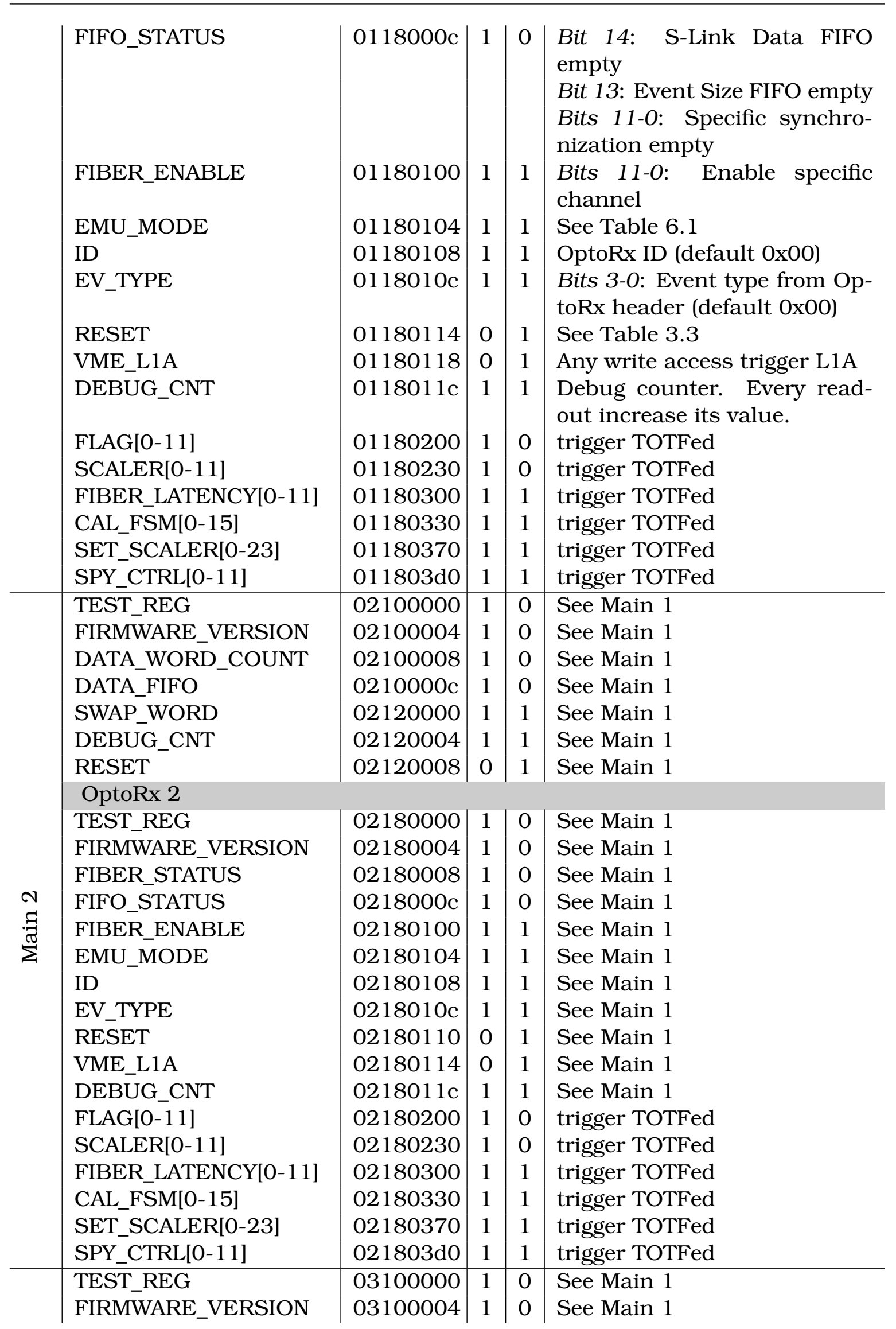




\begin{tabular}{|c|c|c|c|c|c|}
\hline & $\begin{array}{l}\text { DATA_WORD_COUNT } \\
\text { DATA_FIFO } \\
\text { SWAP_WORD } \\
\text { DEBUG_CNT } \\
\text { RESET }\end{array}$ & $\begin{array}{l}03100008 \\
0310000 \mathrm{c} \\
03120000 \\
03120004 \\
03120008\end{array}$ & $\begin{array}{l}1 \\
1 \\
1 \\
1 \\
0\end{array}$ & $\begin{array}{l}0 \\
0 \\
1 \\
1 \\
1\end{array}$ & $\begin{array}{l}\text { See Main } 1 \\
\text { See Main } 1 \\
\text { See Main } 1 \\
\text { See Main } 1 \\
\text { See Main } 1\end{array}$ \\
\hline & $\begin{array}{l}\text { OptoRx } 3 \\
\text { TEST_REG } \\
\text { FIRMWARE_VERSION } \\
\text { FIBER_STATUS } \\
\text { FIFO_STATUS } \\
\text { FIBER_ENABLE } \\
\text { EMU_MODE } \\
\text { ID } \\
\text { EV_TYPE } \\
\text { RESET } \\
\text { L1A_VME } \\
\text { DEBUG_CNT } \\
\text { FLAG[0-11] } \\
\text { SCALER[0-11] } \\
\text { FIBER_LATENCY[0-11] } \\
\text { CAL_FSM[O-15] } \\
\text { SET_SCALER[0-23] } \\
\text { SPY_CTRL[0-11] }\end{array}$ & $\begin{array}{l}03180000 \\
03180004 \\
03180008 \\
0318000 \mathrm{c} \\
03180100 \\
03180104 \\
03180108 \\
0318010 \mathrm{c} \\
03180110 \\
03180114 \\
0318011 \mathrm{c} \\
03180200 \\
03180230 \\
03180300 \\
03180330 \\
03180370 \\
031803 \mathrm{dO} \\
\end{array}$ & $\begin{array}{l}1 \\
1 \\
1 \\
1 \\
1 \\
1 \\
1 \\
1 \\
0 \\
0 \\
1 \\
1 \\
1 \\
1 \\
1 \\
1 \\
1 \\
\end{array}$ & $\begin{array}{l}0 \\
0 \\
0 \\
0 \\
1 \\
1 \\
1 \\
1 \\
1 \\
1 \\
1 \\
0 \\
0 \\
1 \\
1 \\
1 \\
1 \\
\end{array}$ & $\begin{array}{l}\text { See Main } 1 \\
\text { See Main } 1 \\
\text { See Main } 1 \\
\text { See Main } 1 \\
\text { See Main } 1 \\
\text { See Main } 1 \\
\text { See Main } 1 \\
\text { See Main } 1 \\
\text { See Main } 1 \\
\text { See Main } 1 \\
\text { See Main } 1 \\
\text { trigger TOTFed } \\
\text { trigger TOTFed } \\
\text { trigger TOTFed } \\
\text { trigger TOTFed } \\
\text { trigger TOTFed } \\
\text { trigger TOTFed }\end{array}$ \\
\hline & $\begin{array}{l}\text { TEST_REG } \\
\text { FIRMWARE_VERSION } \\
\text { DATA_WORD_COUNT } \\
\text { DATA_FIFO } \\
\text { TRIGG_SCALER[0-12] } \\
\text { TRIGG_LATENCY[0-5] } \\
\text { TRIGG_BIT_PATT[0-5] } \\
\text { TRIGG_DEBUG[0-15] } \\
\text { TRIGG_SPARE }\end{array}$ & $\begin{array}{l}06100000 \\
06100004 \\
06100008 \\
0610000 c \\
06140000 \\
06160000 \\
06160018 \\
06160030 \\
06160070\end{array}$ & $\begin{array}{l}1 \\
1 \\
1 \\
1 \\
1 \\
1 \\
1\end{array}$ & $\begin{array}{l}0 \\
0 \\
0 \\
1 \\
1 \\
1 \\
1\end{array}$ & $\begin{array}{l}\text { Fixed pattern: 0xOBADFACE } \\
\text { Firmware version, } \\
\text { current: Ox } 101 \\
\text { reserved } \\
\text { reserved } \\
\text { trigger TOTFed } \\
\text { trigger TOTFed } \\
\text { trigger TOTFed } \\
\text { trigger TOTFed } \\
\text { trigger TOTFed }\end{array}$ \\
\hline 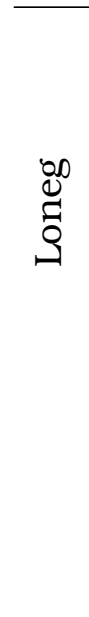 & $\begin{array}{l}\text { TEST_REG } \\
\text { FIRMWARE_VERSION } \\
\text { TRIGG_FIFO } \\
\text { TRIGG_SCALER[0-15] } \\
\text { TRIGG_FED } \\
\text { LATENCY[0-5] } \\
\text { TRIGG_SCALER } \\
\text { SET[0-31] } \\
\text { TRIGG_PRESCALER } \\
\text { SET[0-15] } \\
\text { TRIGG_SET[0-31] } \\
\text { DEBUG }\end{array}$ & $\begin{array}{l}05100000 \\
05100004 \\
05140000 \\
05140004 \\
05160000 \\
05160018 \\
051600 d 8 \\
05160118 \\
05160198\end{array}$ & $\begin{array}{l}1 \\
1 \\
1\end{array}$ & $\begin{array}{l}0 \\
0 \\
1\end{array}$ & $\begin{array}{l}\text { Fixed pattern: OxOBADFACE } \\
\text { Firmware version, } \\
\text { current: OxCOO } \\
\text { trigger TOTFed } \\
\text { trigger TOTFed } \\
\text { trigger TOTFed } \\
\text { trigger TOTFed } \\
\text { trigger TOTFed } \\
\text { trigger TOTFed } \\
\text { trigger TOTFed }\end{array}$ \\
\hline
\end{tabular}


Table 4.5: The registers address mapping in the VME FPGA. To obtain the VME address, the offset should be added to a geographical address of the TOTFed (Section 3.3), for example address of the watchdog from the VME, for the TOTFed located in slot 8 is 0x40000098. Columns $\mathrm{R}$ and $\mathrm{W}$ indicates respectively, if register allows for a readout and a write.

\section{Example usage}

Figure 4.11 presents the working VMECnt entity. The 4.11 shows readout of the Firmware Version registers of the VME (195 ns) and the Main (261 ns). The waveform in Figure 4.12 presents a write access to the Test Register of the VME (195 ns) and the Debug counter of the Main (261 ns). The figures includes signals for Texas's buffers (SN74ABT1645), which protect the Local and VME bus. One should notice that access to the VME register is faster than the Main. However, there is no timing difference between readout and write transfers. 


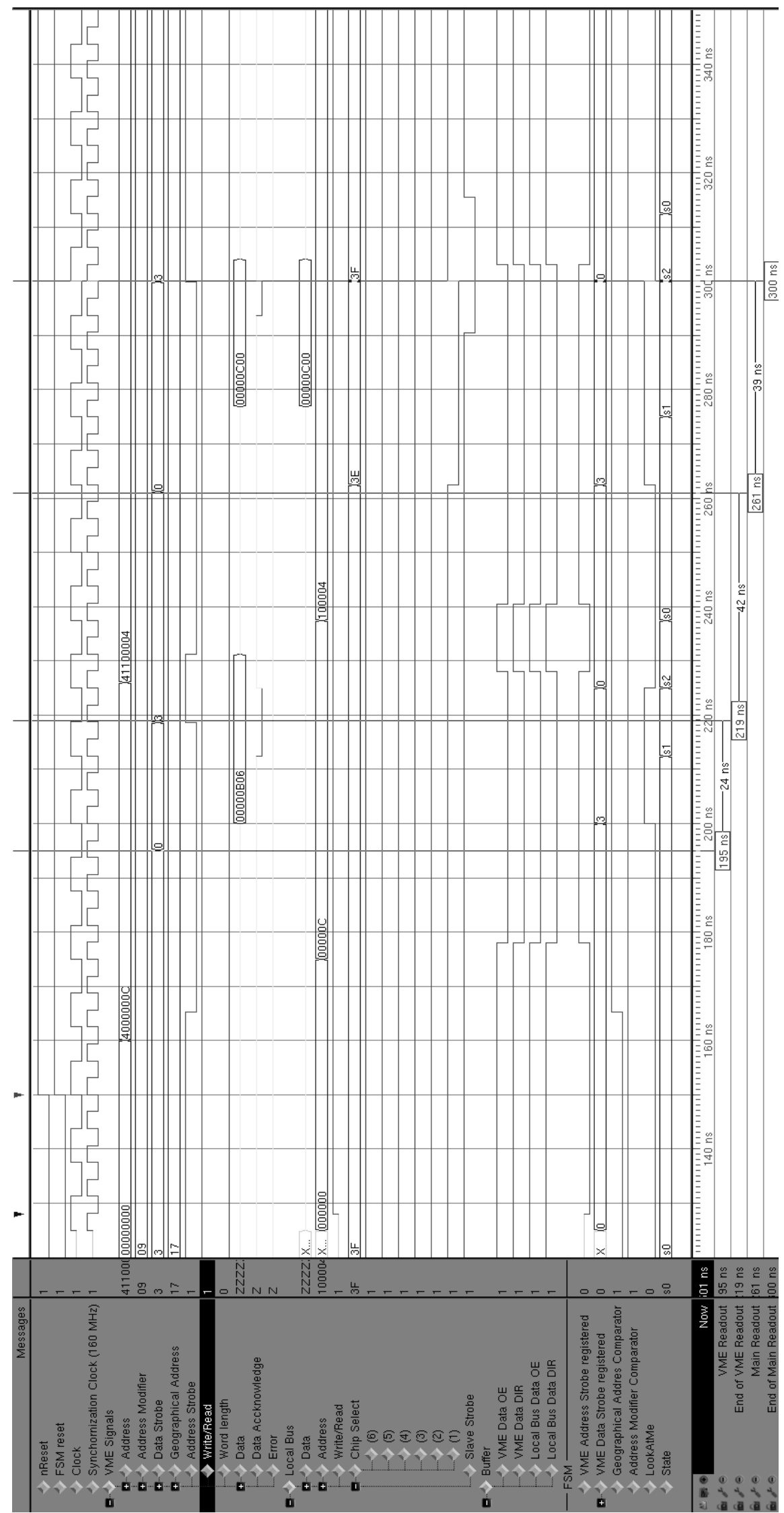

Figure 4.11. Waveforms presenting readout through VMECnt entity. 


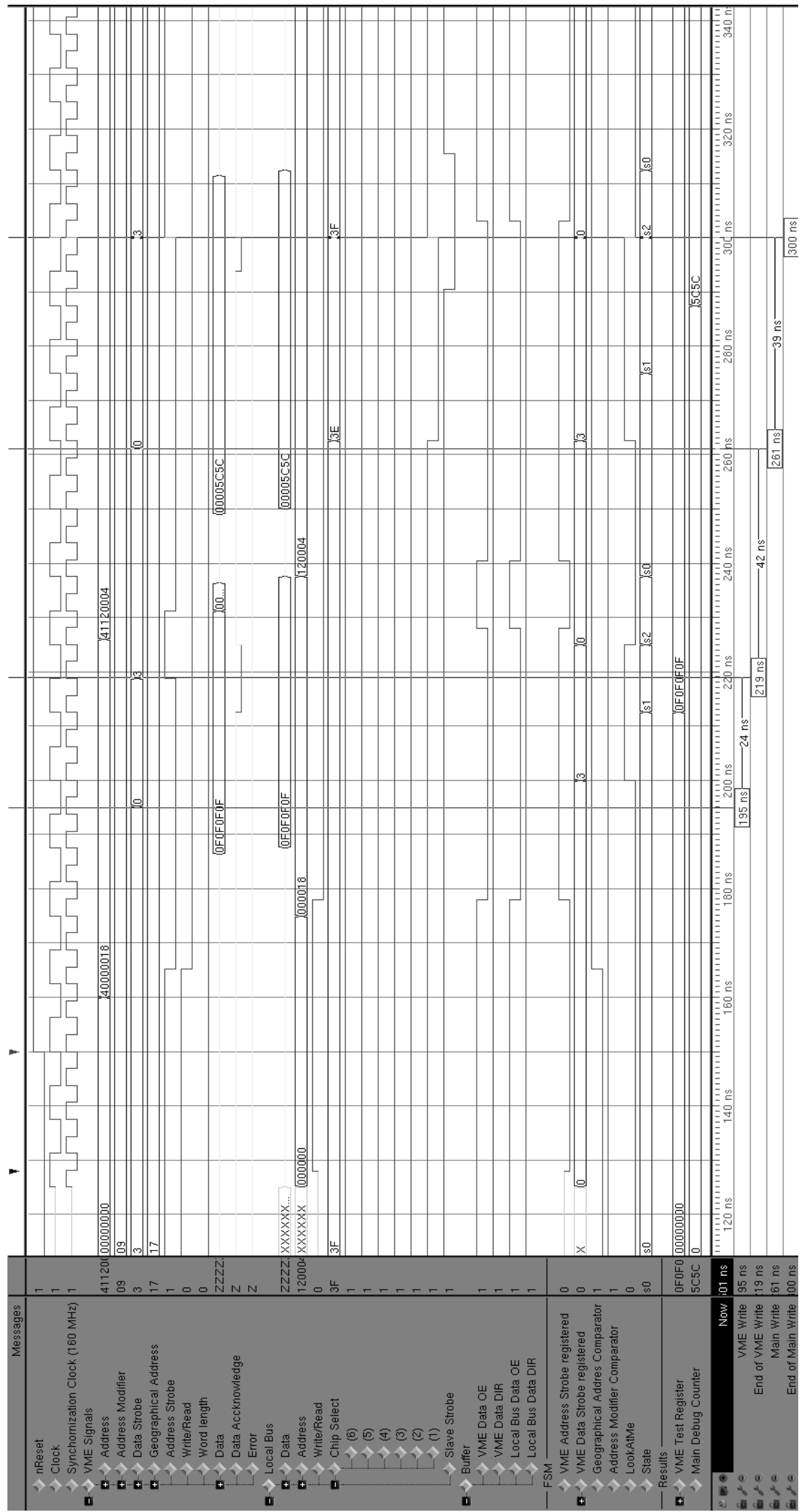

Figure 4.12. Waveforms presenting write through VMECnt entity. 


\section{The Main}

\subsection{General overview}

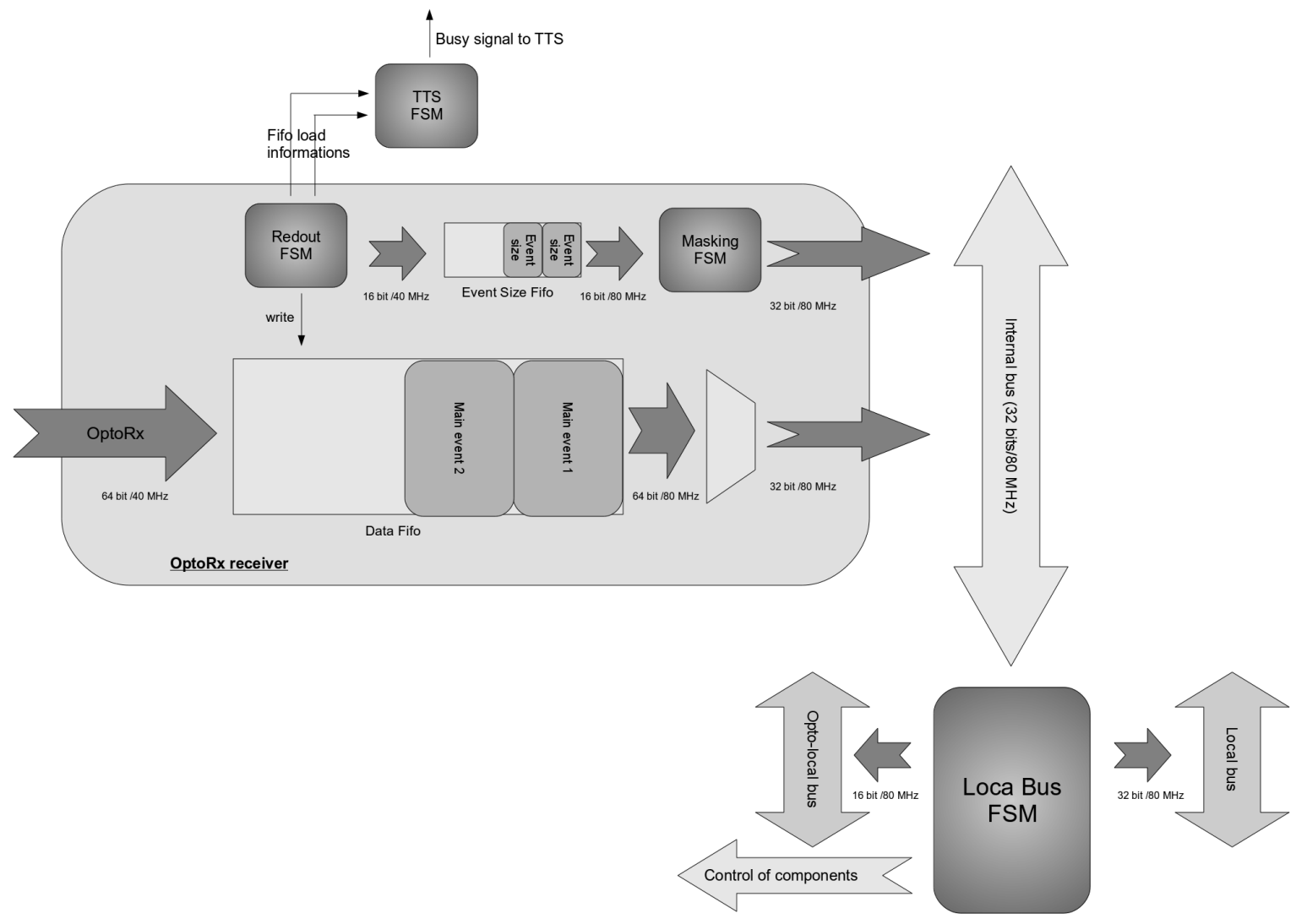

Figure 5.1. The functional scheme of the Main.

The Main uses the FPGA, which belongs to Altera's Startix Family: EP1S20F780C7. This is a well-equipped device, designed for a digital processing, that is capable to satisfy requirements of most applications. The producer characterizes it, as "low-power high-performance". Stratix family is developed at a $130-\mathrm{nm}$ process node and is supplied from a $1.5 \mathrm{~V}$ voltage source. Today such features seem to be a bit archaic, especially if one compares them to the process node of top processors, however during design development the author have not been obstructed by any device timing limitations. The FPGA used as the Main has $18460 \mathrm{LEs}$, about 1,6 Mb of an internal memory, 10 DSP blocks, 80 fast embedded multipliers and 6 PLLs. What is important, it allows to use 586 user's pins, what 
is crucial, for a device, which links several parallel buses. The specific information are available in producer's manual [20]. The logic element in the Stratix looks identically as in Cyclone (Figure 4.1. The speed grade of the used FPGA is 7, what means that it is the one of the slowest in the family.

The Main is a device, which mediate between the VME and the OptoRx. Its main goals are:

- receive data through S-Link interface in the CMS FED Common Data Format (Figure 2.8) and buffer them for a readout via the VME interface;

- allow access to the registers of the OptoRx from the Local bus via so called the Local-opto bus interface;

- generate the TTS signals (Section 2.3.4).

Figure 3.1 shows that each Main has to its disposal $18 \mathrm{MB}$ of the SRAM memory providing No Bus Delay Architecture (NoBL) and the USB2.0 port. This features are not supported by the current version of the firmware, however there are plans to implement needed controllers in next releases. It will give the possibility to enlarge space of the FIFOs and enables the local data readout through the fast interface ${ }^{1}$

The functional scheme of the Main is introduced in Figure 5.1.

\subsection{Implementation}

\subsubsection{The Local bus slave controller}

The Local bus interface should offer the data rate between the VME and the Main, which satisfy the $1 \mathrm{kHz}$ experiment trigger rate (L1A) requirement. Implementation of an effective data exchange protocol between above parts of the project had faced with some problems. The reason was the fact, that when the TOTFED board had been designing, specification of the Local bus was not fully thought out. It was assuming, that data should be available after a fixed time. Of course the time had to take account the worst case - a delay of a slowest device. This meant that the interface was slow and unreliable. What is more, any later efforts to improve performance met with a problem of deficiency of electrical lines. The current version of the Local bus should use as least as possible control lines. A data word size is 32 bit and the interface uses 24-bit addressing. As the original project, the bus remained asynchronous.

The author decided to use three control signals. The example data transfer shows Figure 5.2 .

As reader can notice, at the beginning the master of the bus (all the time this role is assigned to the VME) prepares the Address and the WR signals. The second of them indicates transfer type: a readout (high) or a write (low). After this, the Local Chip Select signal is asserted (active low) by the master. Next, the slave of the bus (one of the three Mains) issues Data. When the data are valid, it asserts the Local Slave Strobe (active low). The Local Chip Select remains low, until the master complete the data readout. Farther, the slave finishes transfer denying the Local Slave Strobe. The author would like to point out, that each slave (the three Mains and the Merger) uses the independent Local Chip Select signals. A lot of troubles were caused by the Local Slave Strobe. Because of an electrical line

\footnotetext{
${ }^{1}$ Usage of the S-Link 64 interface is possible only within the CMS system.
} 


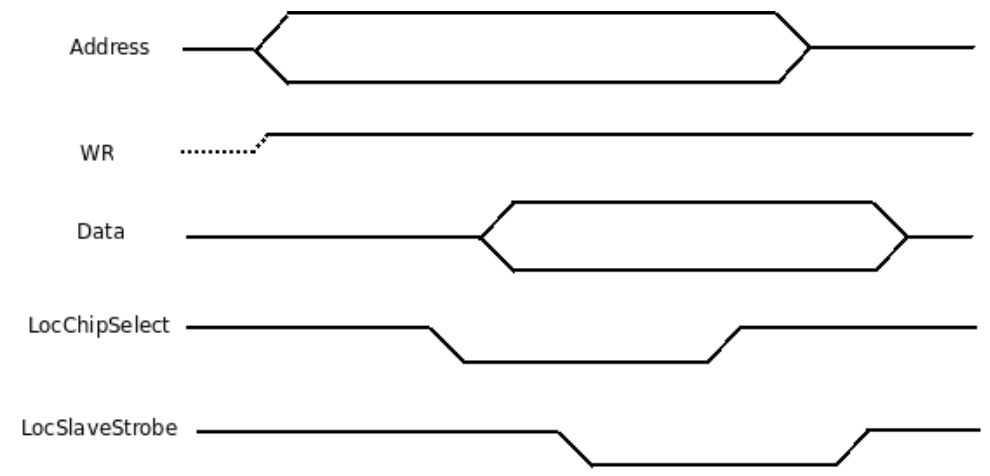

Figure 5.2. The example readout of the Main by the VME using the Local bus interface.

deficiency, this signal has to be shared by all slaves. That approach forces to use a high impedance and pull-up resistors. The layout of the TOTFed board does not consider this fact, so in the project internal weak pull-up resistors of the FPGA are used. Naturally a solution, which rely on pull-up resistors ${ }^{2}$ is slower than a direct transistor-transistor communication (TTL). For this reason the state machine uses some kind of trick to gain performance.

The declaration of the entity is presented below. It uses generic style of coding, because the module is common for the Main, the Merger (the Loacl Bus) and the OptoRx (the Opto-Local bus).

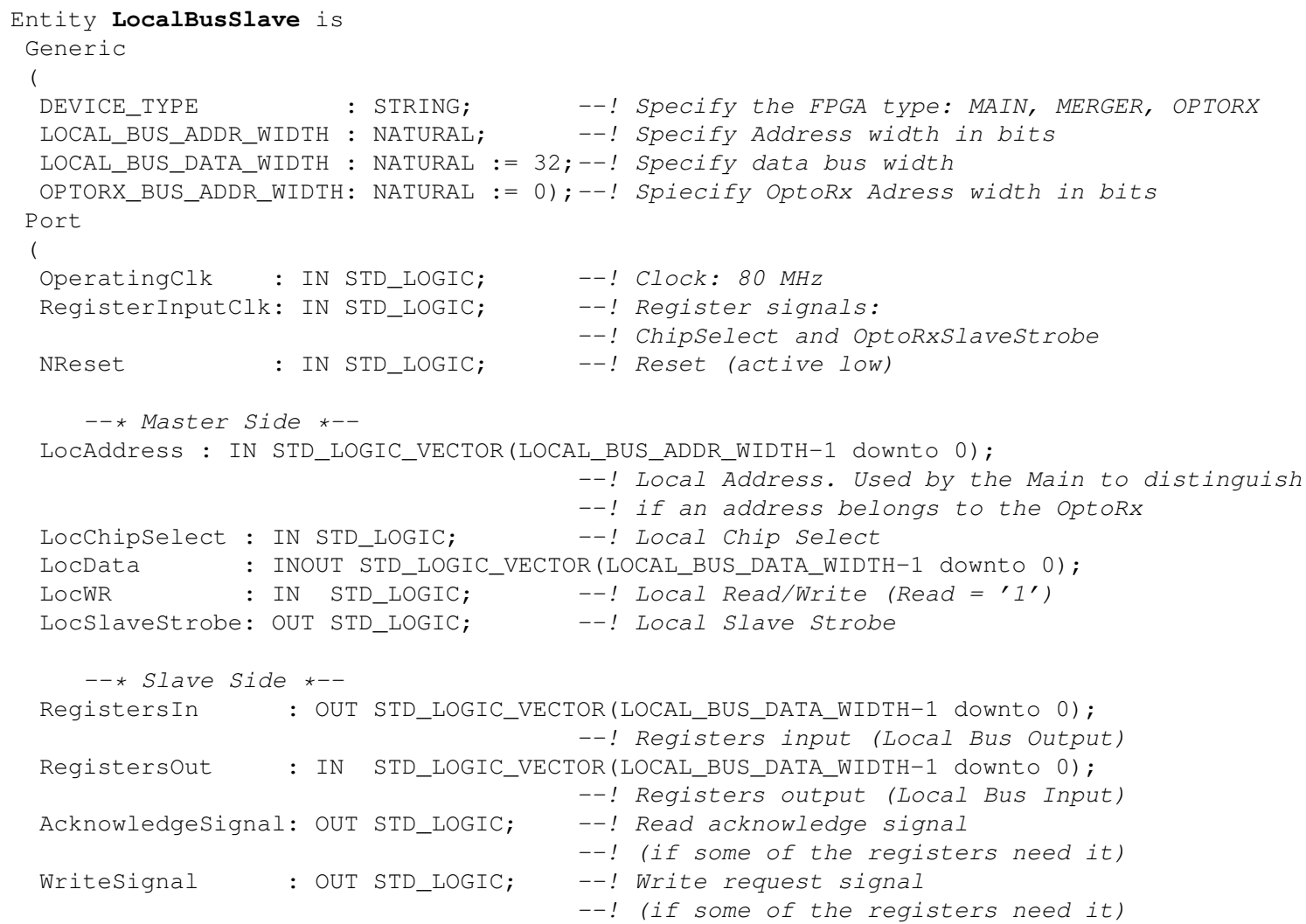

\footnotetext{
${ }^{2}$ which, in addition, are not discreet board elements
} 


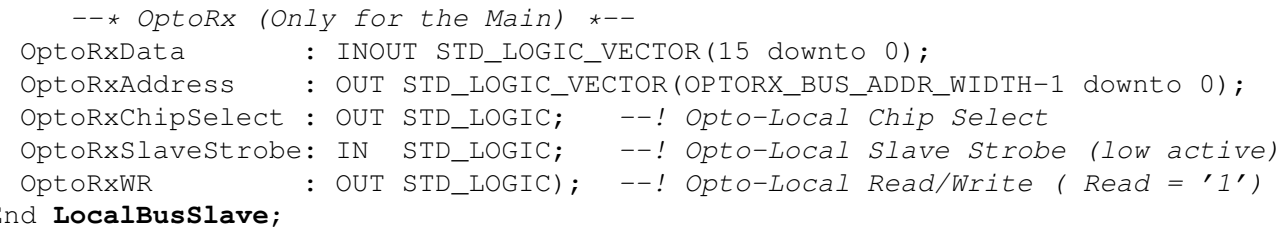

The scheme of the of the Local bus slave controller is showed in Figure 5.3 . The major part of the module is a state machine. From the same reasons as the VME Controller from the VME FPGA 4.2.3 it uses Mealy approach. Similarly, the critical signals from performance point of view (the OptoRx slave strobe and the LocChipSelect), to avoid glitches, are sampled by flip-flops clocked with $160 \mathrm{MHz}$. The rotting tool of Quartus II knowns clocks of components specified in '.sdc' file so it will take care of a timing requirements between marked on red the flip-flops from the figure and rest of the components. All multiplexers from the module use as a select signal the addressType. It is ' 1 ' when address correspond to the OptoRx (Table 4.5). The supported interfaces, the Local Bus and the Opto-local bus, are bidirectional, so data paths contain tri-state buffers driven by the state machine.

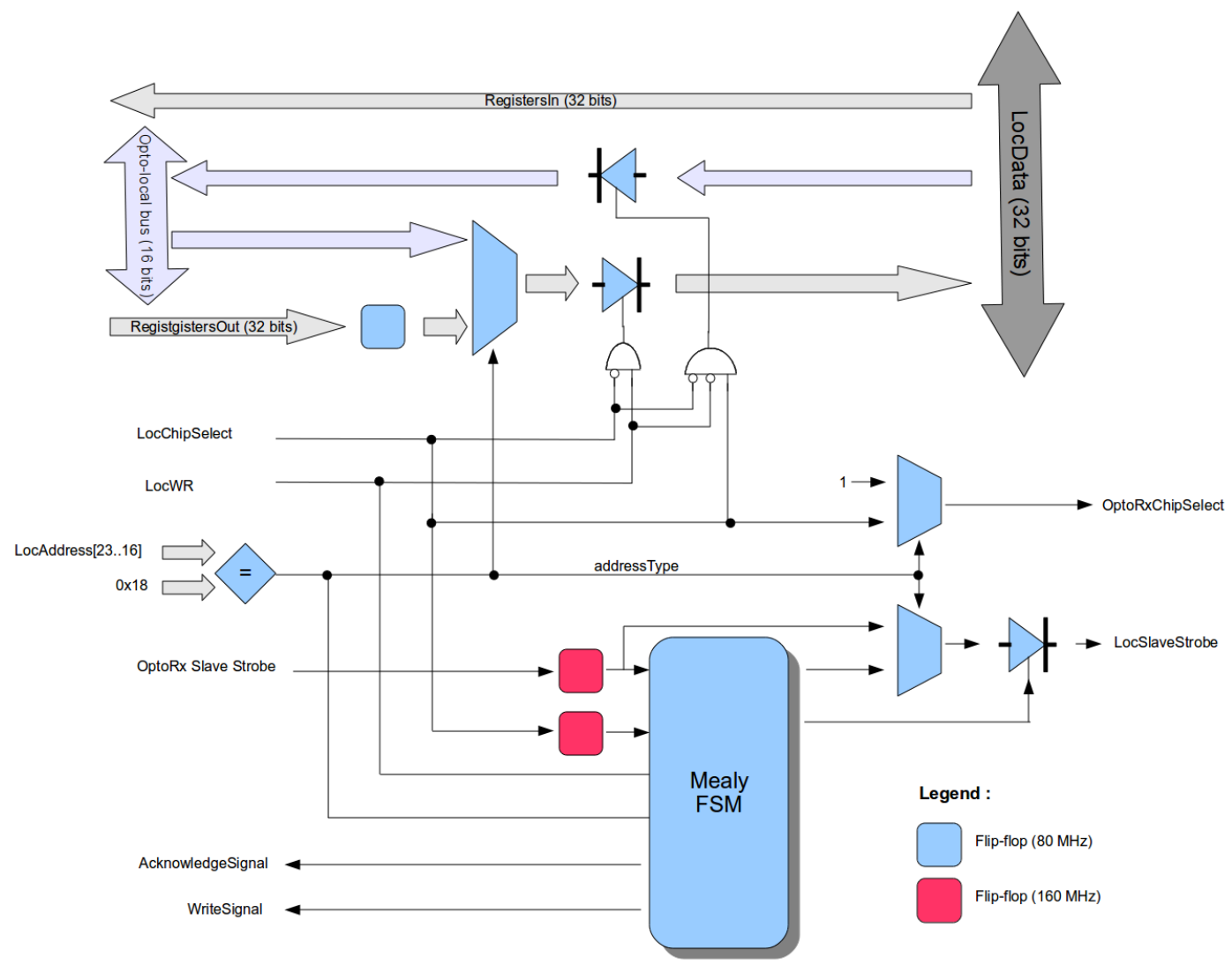

Figure 5.3. Scheme of the Main Local Bus slave Controller.

The output of the Main registers is connected to the RegistersOut and the input to the RegistersIn. The RegisterOut is routed through a register to cut the logic path from an external tree of multiplexers which selects the source of data. Some registers can need the read acknowledge (for example look-ahead FIFOs) or the write request signal (setting registers). They are delivered by the Mealy FSM and 
multiplexed outside the module to appropriate recipients. Signals are active high by one $80 \mathrm{MHz}$ clock cycle. For this reason, front-ends of the rest modules should use the same clock to avoid a timing violence.

\section{State machine}

As it was mentioned, the Local bus slave is controlled by Mealy's finite state machine. Figure 5.4 presents the states transitions.

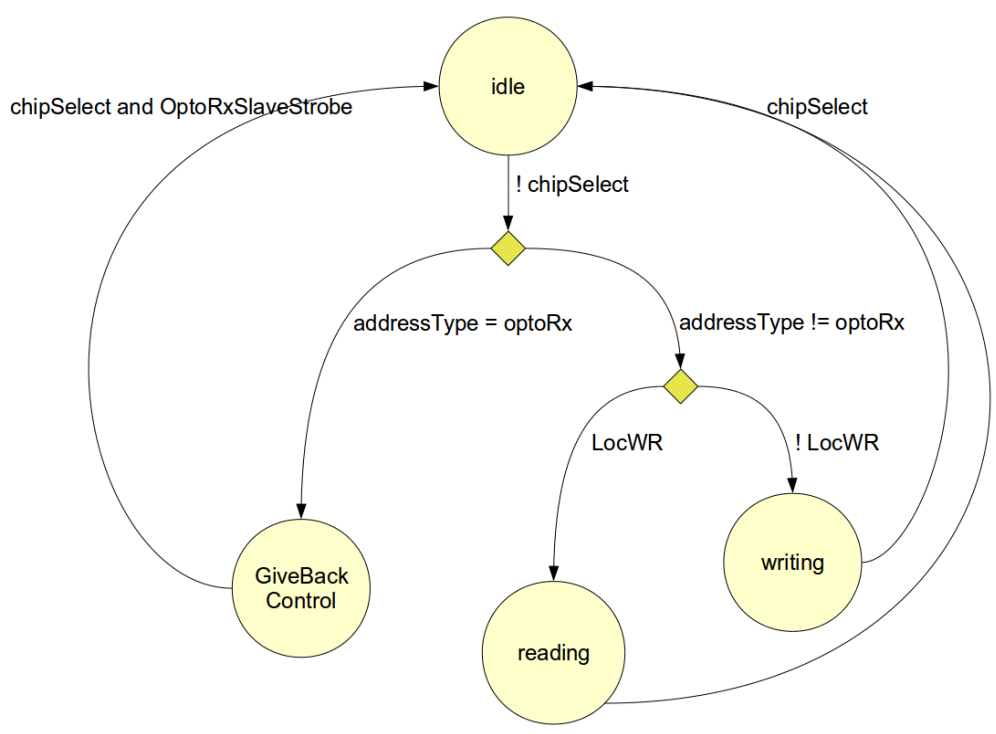

Figure 5.4. States transition for Mealy's finite state machine controlling the Local Bus slave interface in the Main.

At the beginning, the FSM waits for an assertion of the LocChipSelect signal (active low) from the latch clocked with $160 \mathrm{MHz}$. At the same time, the LocAddress is compared with the mask corresponding to the address space of the OptoRx. The result drives the addressType line. If it is ' 1 ', the state machine goes to the giveBackControl state. All control and data lines are driven in this state by the OptoRx. The FSM simply waits for the transmission end pointed by an assertion of the OptoRxSlaveStrobe by the OptoRx and a deny of the ChipSelect by the VME FPGA . After that it comes back to the idle state.

In other case, if LocAddress belongs to the internal registers of the Main, the state machine checks the LocWR signal. In case of a readout ( $\operatorname{Loc} W R=$ ' 1 ') the state machines, as it is presented by the diagram, goes to the reading state. It asserts the SlaveStrobe signal (active low) and enables the tri-state buffer related to the LocData. Data are already valid, because correct address came from the VME bus muster, routed throughout the VMA FPGA, a few cycles before $(12.5-25 \mathrm{~ns})$. The multiplexers had a time to switch itself. In the reading state the FSM waits for the end of transaction which is indicated by a deny of the ChipSelect by the VME FPGA. As the SlaveStrobe line uses the weak pull-up resistors, the time of a charge reload through their resistance is long. It is possible a scenario, where the VME would make subsequent accesses ${ }^{3}$ and the SlaveStrobe would not have a time to be loaded to a high value. The VME would consider invalid data as correct, because the state

\footnotetext{
${ }^{3}$ for example in the block readout mode
} 
of the SlaveStrobe would indicate a logic zero. For this reason, in the reading state, as soon as the state machine notices deny of the ChipSelect it starts driving the SlavStrobe to high value through a transistor. The FSM goes to the idle state only on a nearest rising edge of the $80 \mathrm{MHz}$ clock after the deny, the tri-state buffers are disabled and the SlaveStrobe remains in a high impedance. That approach lets to obtain fast edges of the signal and sorted out the interpretation problem on Local bus's master side. Furthermore, at the same time, when FSM starts driving the SlaveStrobe to high it asserts the read acknowledge signal. It is enabled on a nearest edge of the clock and can be interpreted by the internal registers.

If in the idle state, the address indicates an internal register of the Main and LocWR='0', the FSM asserts the write request signal. On a next clock edge it goes to the writing state, drives the SlaveStrobe to logic zero and waits for the end of transmission from the VME FPGA side. Driving of the SlaveStrobe after ChipSelect deny is made on the same way as for the reading state.

\section{Multiplexers tree}

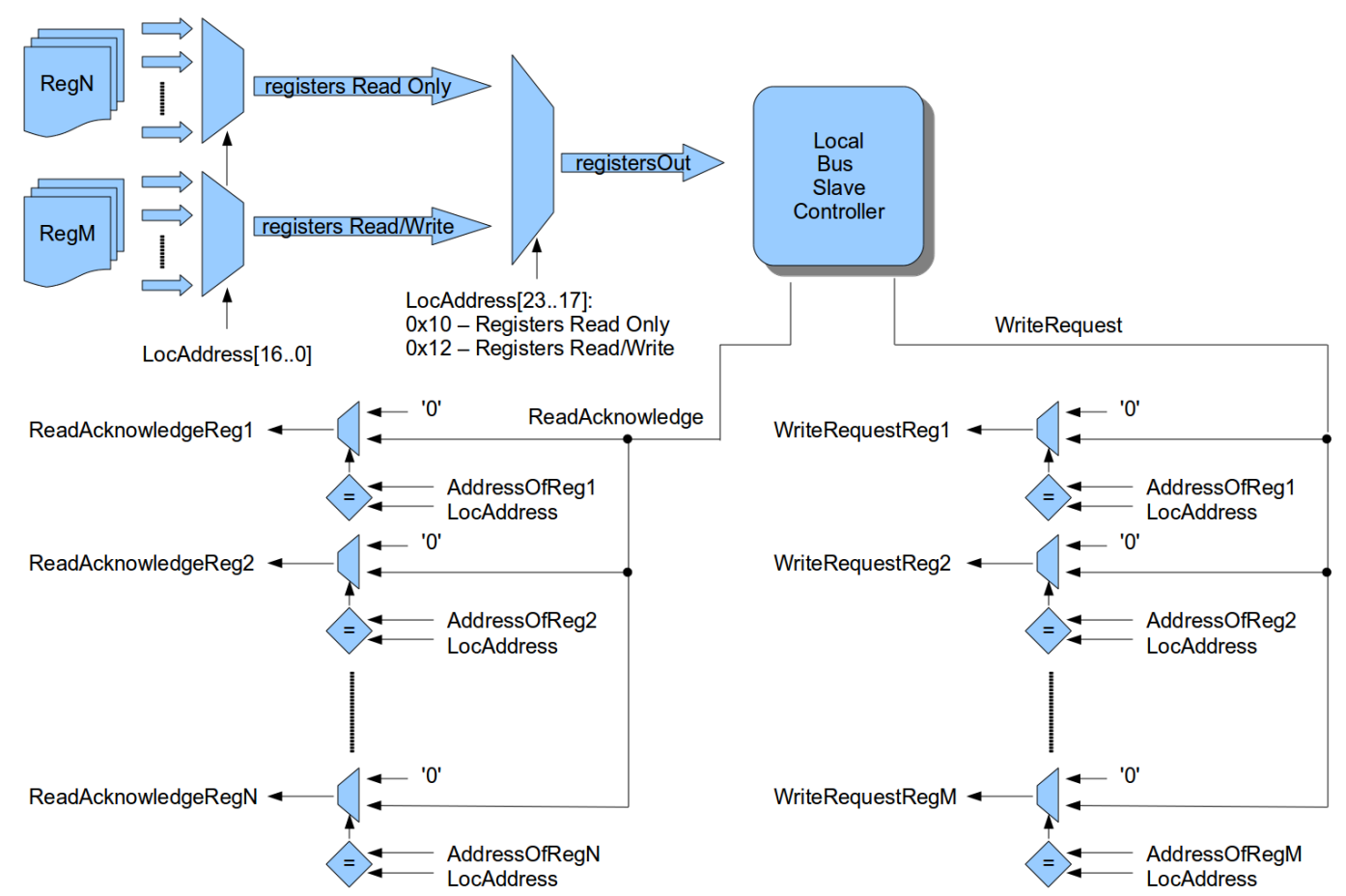

Figure 5.5. The structure of the multiplexers tree of internal registers related to the Local bus slave controller in the Main.

Outside the entity managing communication with the Local bus, there exists a tree of multiplexers presented in Figure 5.5. According to Section 3.3 the FPGAs uses some kind of the memory paging. The registers in the Main can be split into two groups: Read Only and Read/Write (respectively RegN and RegM in the figure). Specific registers is chosen by 17 least significant bits of the LocAddress. The next level of the multiplexers, using seven most significant bits of the LocAddress, selects the appropriate group of the registers. If in the future design will need new 
set of registers related to new objective 4 , it can be easily connected to a following level of the multiplexers tree.

As it was written above, a part of the registers can require the write request or, for some reasons, the readout acknowledge signal5. They are generated by the state machine in the Local Bus Slave Controller entity and are routed to all recipient by the hierarchical structure showed in Figure 5.5. One register can use two types of signal at the same time. The only obligation, which it has to satisfy, is the usage of the same $80 \mathrm{MHz}$ clock as the FSM from Figure 5.4. It allows avoid timing violence. As at present the number of registers is much lower than a theoretical limit related to the LocAddress, there is no need to built one big decoder common for all registers ${ }^{6}$. Instead of that, each register has an independent comparator. This approach in totally uses less resources than mentioned.

\section{Example usage}

Figure below presents usage of the Local bus slave controller. The waveform in Figure 5.6 shows readouts of the Firmware Version Registers of the Main (207 ns) and the OptoRx (282 ns), while 5.7 writes to their Debug counters. Naturally an access to the OptoRx FPGA lasts longer, however there is no a timing difference between a write and a readout transfer.

\footnotetext{
4 for example, project will be used in the trigger TOTFed

5 for example a look-ahead data FIFO

${ }^{6}$ this decoder would need to use all bits of the LocAddress, because addresses are scattered
} 


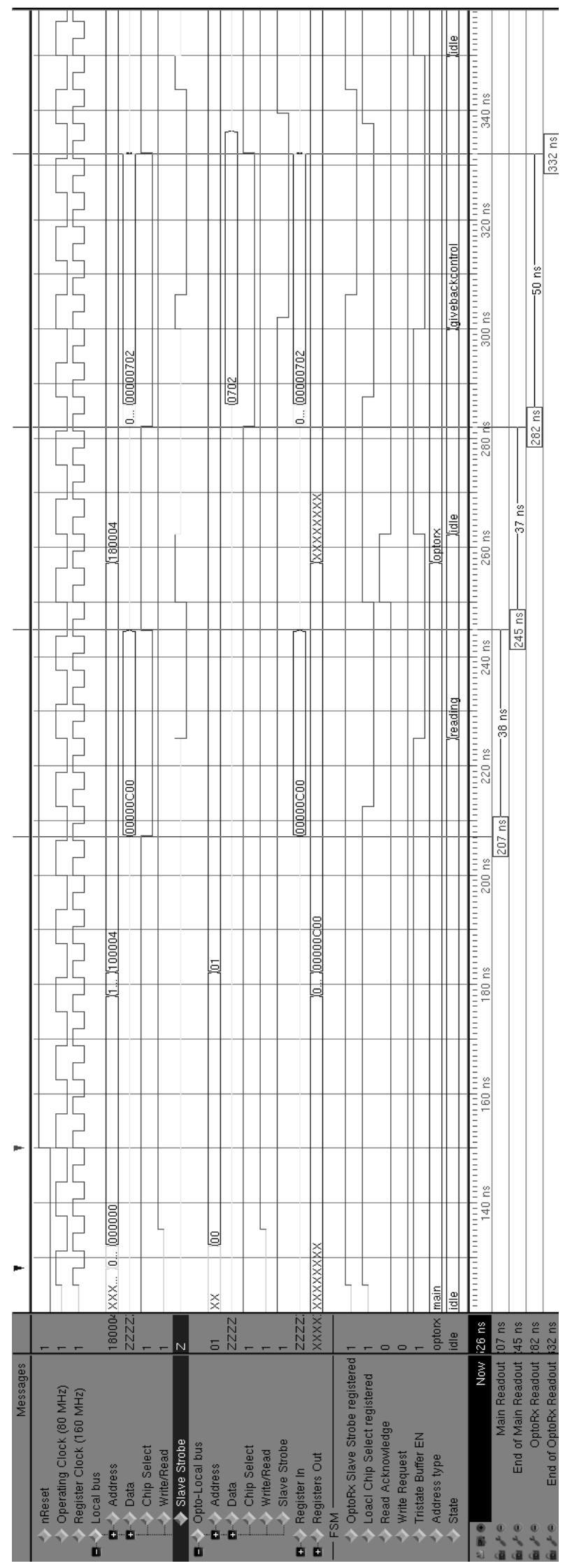

Figure 5.6. Waveforms presenting readout of the Local bus slave controller. 


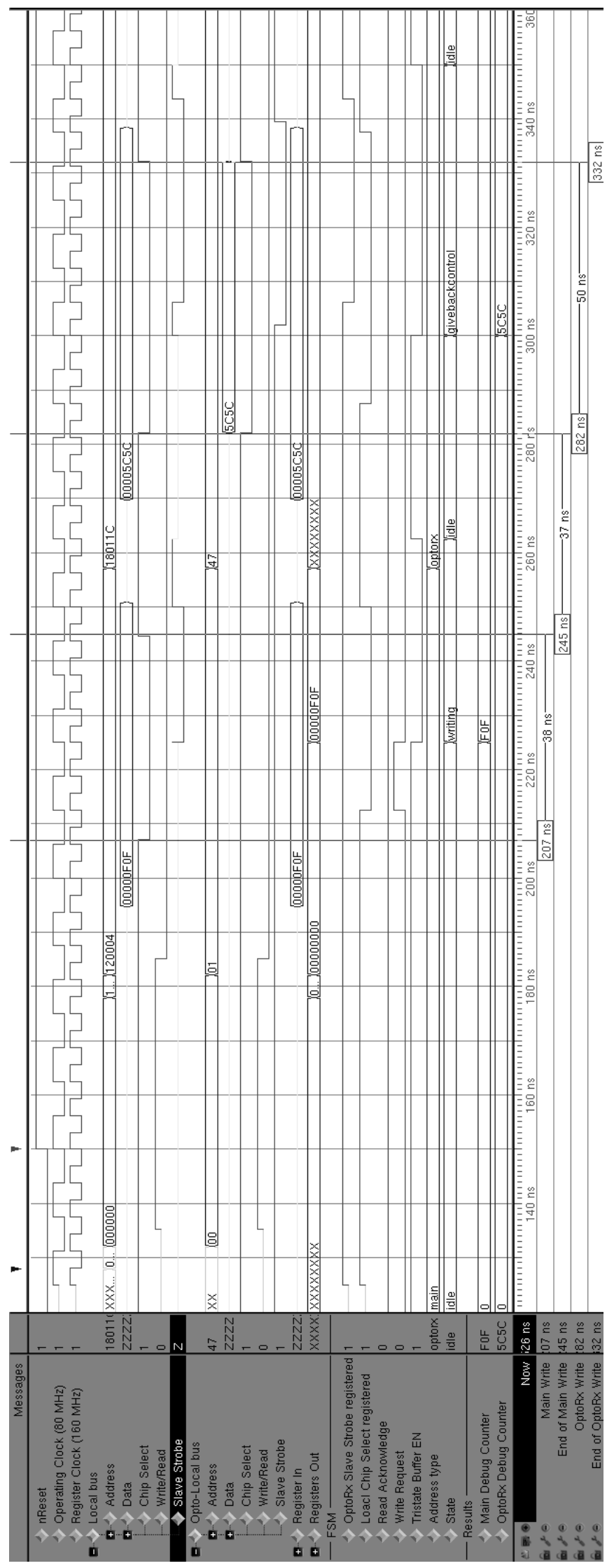

Figure 5.7. Waveforms presenting write access to the Local bus slave controller. 


\subsubsection{The OptoRx receiver}

The OptoRxReceiver is the major part of the Main FPGA. It assembles data from the OptoRx buffering and enables their readout from the VME bus level. Below is presented the entity declaration:

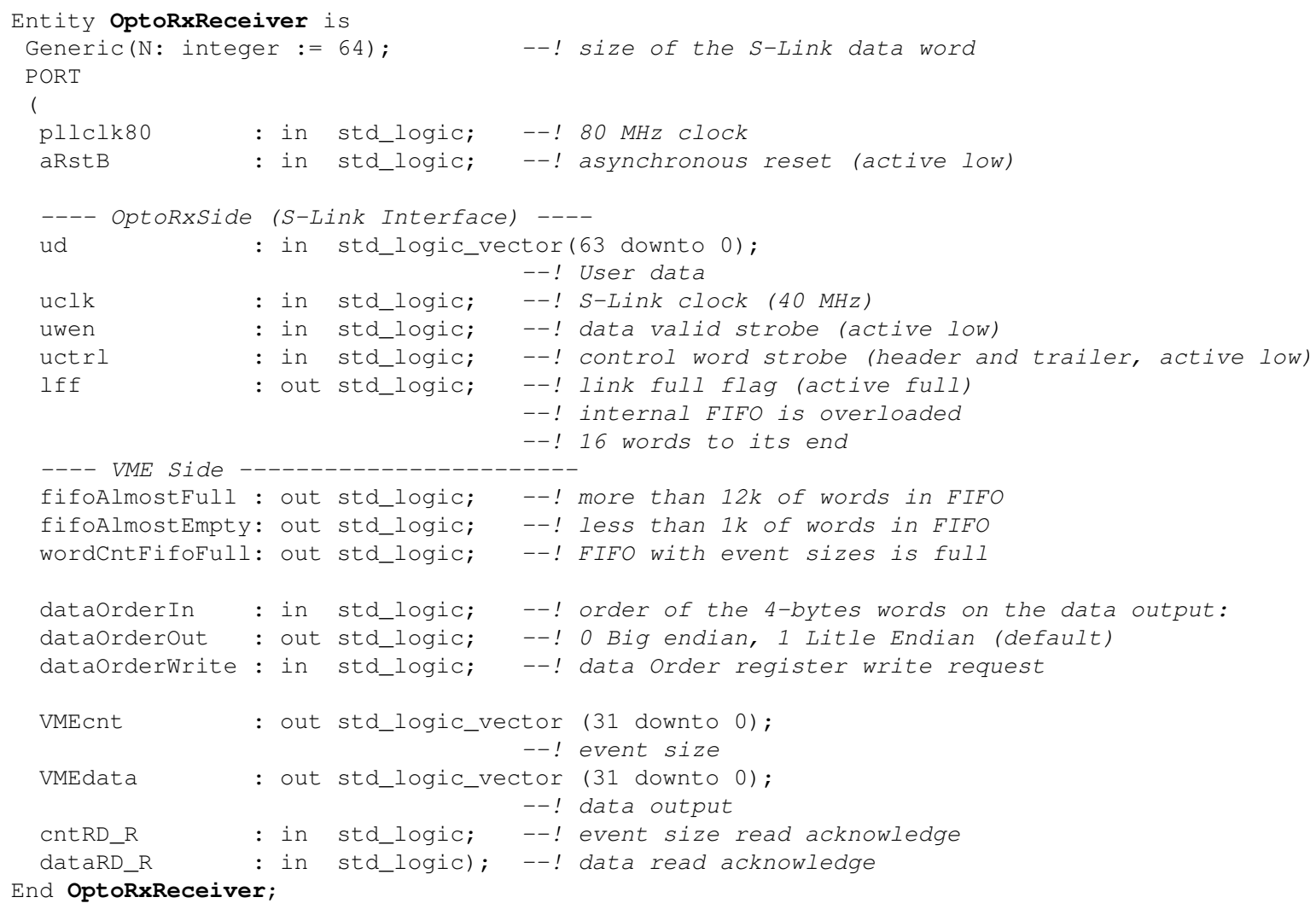

Figure 5.8 presents the block scheme of the OptoRxReceiver. The entity uses two clock domains: one clocked with $40 \mathrm{MHz}$ provided by a local S-Link $\mathrm{f}^{7}$ interface, marked in the figure by the blue rectangle and the second clocked with 80 $\mathrm{MHz}$ generated by an internal PLL of the FPGA. A clock bride is implemented as dual-port FIFOs. The coming 64 bits of the user data goes to the Data FIFO capable to store 16k of 64 bits words. With the Data FIFO are related 3 kinds of flags:

- FIFO almost empty - asserted if in the FIFO is less than $1 \mathrm{k}$ words;

- FIFO almost full - asserted if in the FIFO is more than $12 \mathrm{k}$ words;

- FIFO FULL - asserted if in the FIFO left space only for 16 words.

The output of the Data FIFO is demultiplexed on the two 32-bits words. According to the data order register, first are sent either the four most significant bytes (Big Endian) or the four least significant bytes (Little Endian - a default value).

The control signals from the local S-Link interface drive the Readout FSM. It provides the write strobes for the Data and Event Size FIFOs and manages the 16-bits word counter. After each S-Link frame the value of the counter is collected in the Event Size FIFO capable to store 256 words. The FIFO generates FIFO full flag if there is no more space to write data. The author would like to point out, that each FIFO has two independent ports: one to read and second to write. The free 11

${ }^{7}$ It is called local S-Link interface because it does not satisfy all requirements of the specification 


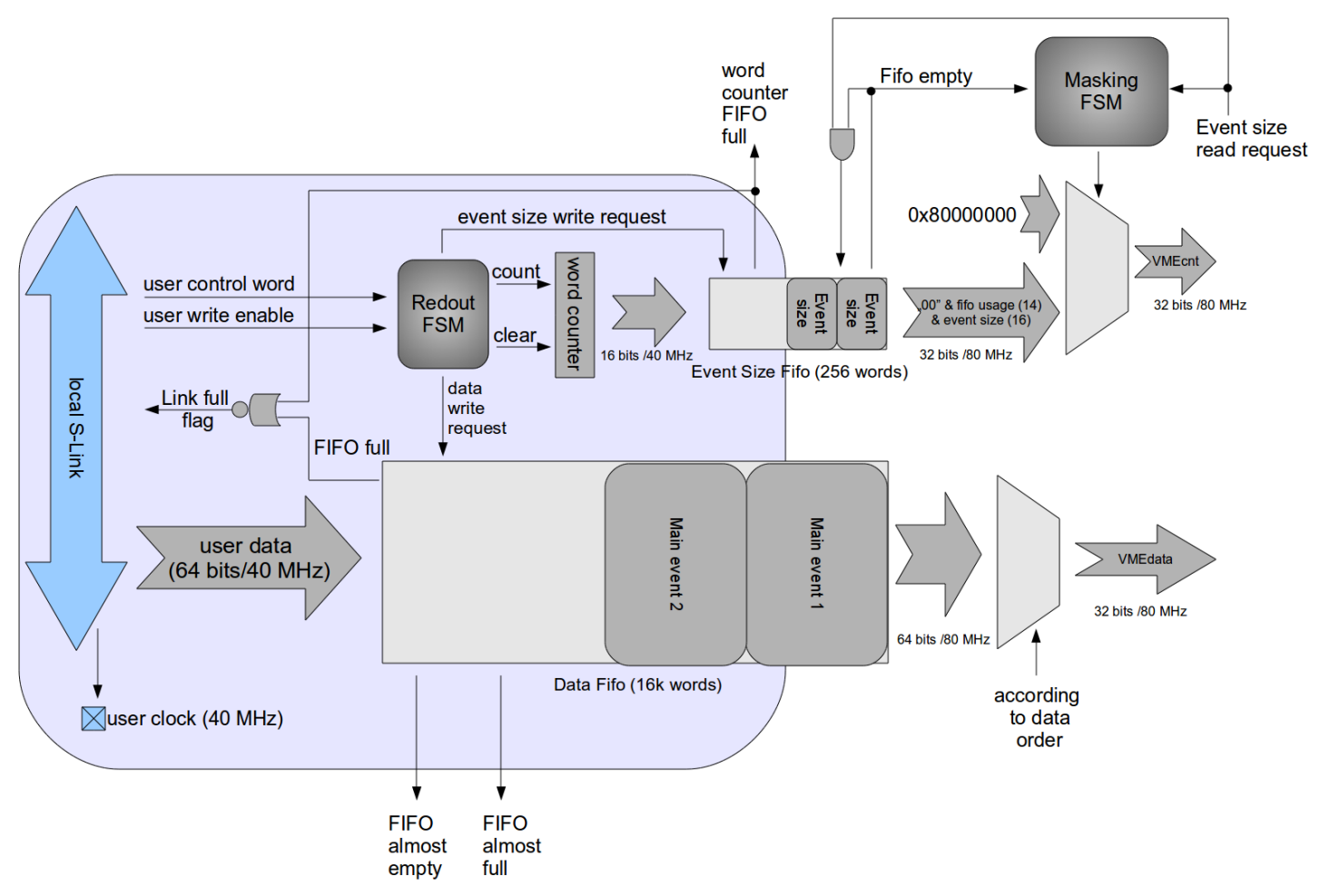

Figure 5.8. The OptoRxReceviver block scheme.

space status can differ on this two ports - incoming data show up on the output after a certain internal delay of the FIFO. All already described flags are related to the write ports. The Data FIFO almost full and the word counter FIFO full flags drive directly the Link Full Flag of the S-Link interface. When it is asserted, the source of data (the OptoRx) should suspend the transfer within the nearest two clock cycles. Thee flags (the word counter FIFO full, the Data FIFO almost full and the Data FIFO almost empty) are used by the component, which generates the TTS information (Section 2.3.4) for the VME FPGA.

The Data FIFO to ensure a quick access to data a use show-ahead mode. It means, that incoming data are issued directly on the output without a read request signal. The FIFO needs only the read acknowledge signal to switch to a next word.

\section{Readout FSM}

The Readout FSM, to assure quick time of reacting and high performance, uses Mealy's approach. The states transitions are introduced by the diagram 5.9 .

The state machine is driven by two low level active signals. The first one, user write enable (UWEN), is asserted when data are valid. The second one, user control word (UCTRL), indicates in the local S-Link implementation a header and a trailer of a data frame. If two of the signals are asserted, the FSM enables the write to the Data FIFO and goes to the payload states. In the payload state the counter counts subsequent words. It can happen that for some reasons (for example active level of the Link Full Flag) the source will suspend the transfer and deny the user write enable. In the FSM this situation is related to the pause state. When master resume the data sending, the state machine will back to the payload state or, in 


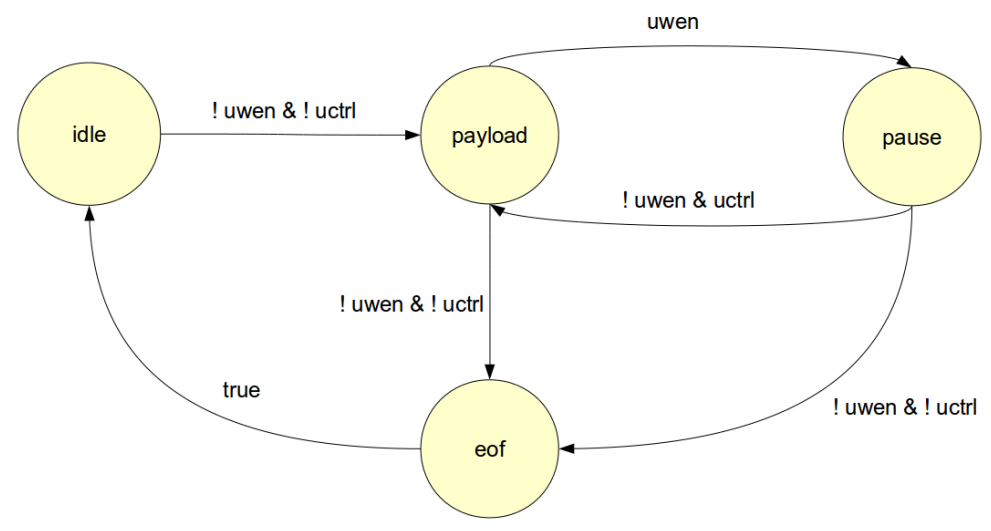

Figure 5.9. The states transitions diagram of the Readout FSM from the OptoRxReceiver entity.

case of the trailer, will go to the eof state. In the eof state, the number of the words of the current S-Link frame is written to the Event Size FIFO. After a one clock cycle the FSM moves back to the idle. In the idle state counter value is cleared and the state machine waits for a next transfer.

\section{Event Size FIFO output}

The output of the Even Size FIFO is a quite elaborate. The software, to start a readout process, pools its value. The FIFO is made of an internal RAM blocks. To be compatible with the Local bus slave controller (Section 5.2.1), it can relay only on the read acknowledge signal. What is more, a FIFO implementation in Quarts II ver. 9.0 is corrupted. Although the FIFO receive the clear signal, output is not driven to default value, which is critical from the software point of view.

The Masking FSM toggles the event counter output between a fixed pattern indicating no events in the Data FIFO and the word which is a combination of the Data FIFO utilization and the size of the first event, as it is presented in Figure 5.8. The Data FIFO usage can be interpreted as a debug information by the software. The most significant bit of the event counter output, if is high, indicates the that there is no events in the Data FIFO ready for a readout.

The state transitions of the Masking FSM are presented in Figure 5.10. It uses Moore's automaton. The FSM starts from the $s 1$ state. Unless the Event Size FIFO is empty, the event size output is driven to the fixed pattern value. After data appears in the FIFO, the FSM expects the read acknowledge signal to switch the FIFO output value (from unspecified to to a first event size). If a read acknowledge signal will come in the same clock cycle as a deny of the FIFO empty the state machine will go directly to the s3. In other case, the FSM will switch to the s2. It means, that even if a event size is already in the FIFO, the first readout will be dummy, because the internal output pointer of the FIFO needs the read acknowledge to increment itself. All this time the output has to be masked. In the s3 state, the FSM drives the output multiplexer to the to combination of the real data from the Event Size FIFO. If in the s3 state the FIFO will be emptied it goes to the $s 4$ state. It waits hear for following data. If it happen, the state machine will back to the $s 3$. However, if during the $s 4$ state the VME will request data, the state 


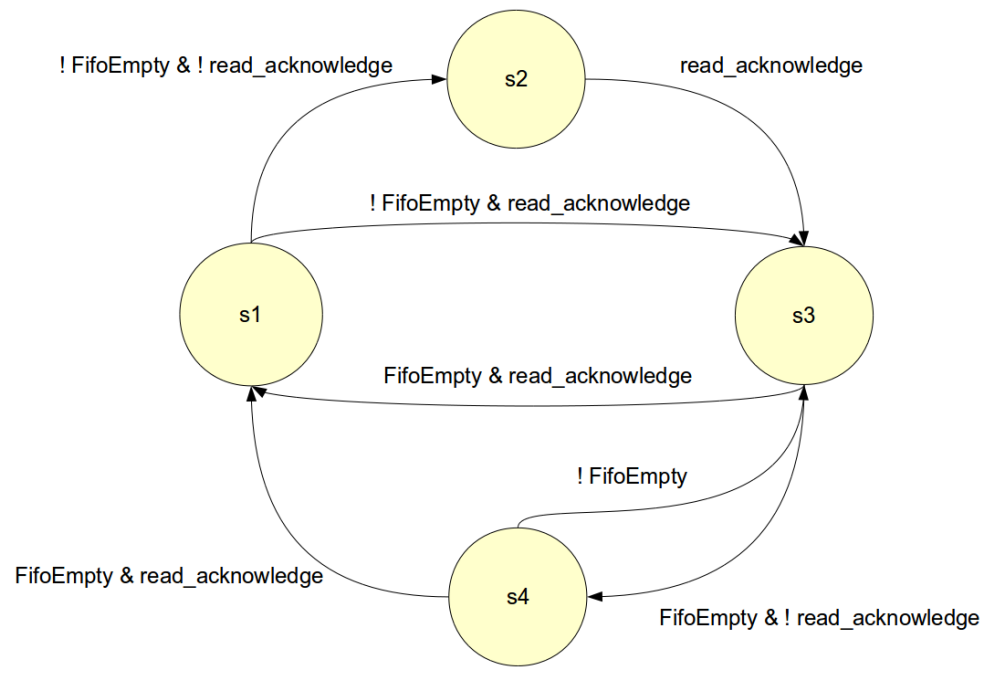

Figure 5.10. The states transitions diagram of the Masking FSM from the OptoRxReceiver entity.

machine will go to the $s 1$ and next data readout, if data will appear, will need a following dummy readout.

\section{Example usage}

Figure 5.11(a) presents the write access to the OptoRx Receiver from the S-Link interface (15 $402 \mathrm{~ns}$ ). The event frame starts with the correct Optorx header (0x5000000000700010) accompanied by asserted UWEN and UCTRL strobe. The FSM goes to the payload state and the word counter starts counting following data words. The event frame 8 lasts $14600 \mathrm{~ns}$ and is finished with the OptoRx header (0xA000024800000080) indicated by active the two S-Link strobes (30 $002 \mathrm{ns).}$ The state machines moves to the eof, writes a counter value to the FIFO and goes back to the idle state expecting a next event.

Figure 5.12 shows readout of the already stored frame. At the beginning (30 $425 \mathrm{ns)}$ the software pools the event size. One should remember, that signal VME Data ack from waveform in Figure 5.12(a) is the read acknowledge from the Local bus slave controller, so even if event is already collected in the FIFO, software, for initial conditions, has to make two access to get a satisfying value because of the masking state machine. As data order is high (little endian) the first VME word corresponds to the least significant bytes of the event header. After the last readout (1 $18188 \mathrm{~ns}$ ) the FIFO output is dummy and will be replaced by correct word after receiving a next event via the S-Link interface.

The last waveform in Figure (5.13) presents behave of the OptoRx Receiver for the FIFO full condition. The FSM asserts the LFF (15 $552 \mathrm{~ns}$ ) and expects a deny of the UWEN, which according to the specification [11], has to occur at most after two clock cycles (in the figure after one). The FSM moves to the pause. As soon as the data FIFO get a free space, the $L F F$ is denied and the S-Link master resume

\footnotetext{
${ }^{8}$ all fibers enable
} 
the transmission (15 $602 \mathrm{~ns}$ ). One should notice the fact, that although the $L F F$ condition, the frame is consistent 9 , what proves the correct work of the entity.

${ }^{9}$ the width of the event frame counts $14450+150=14600 \mathrm{~ns}$ which was the same for the one from Figure 5.11 


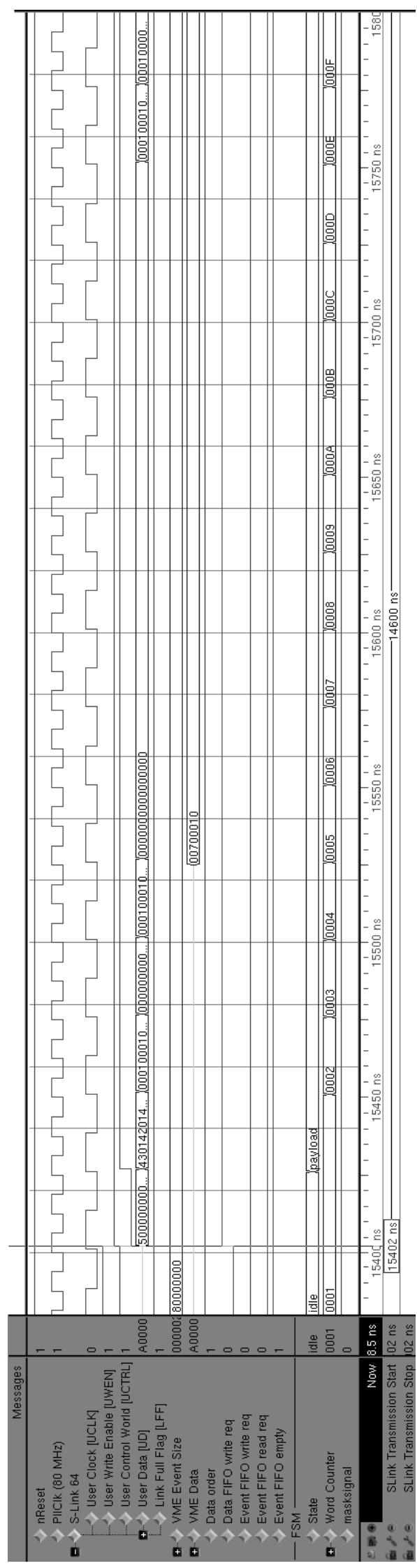

(a) Beginning

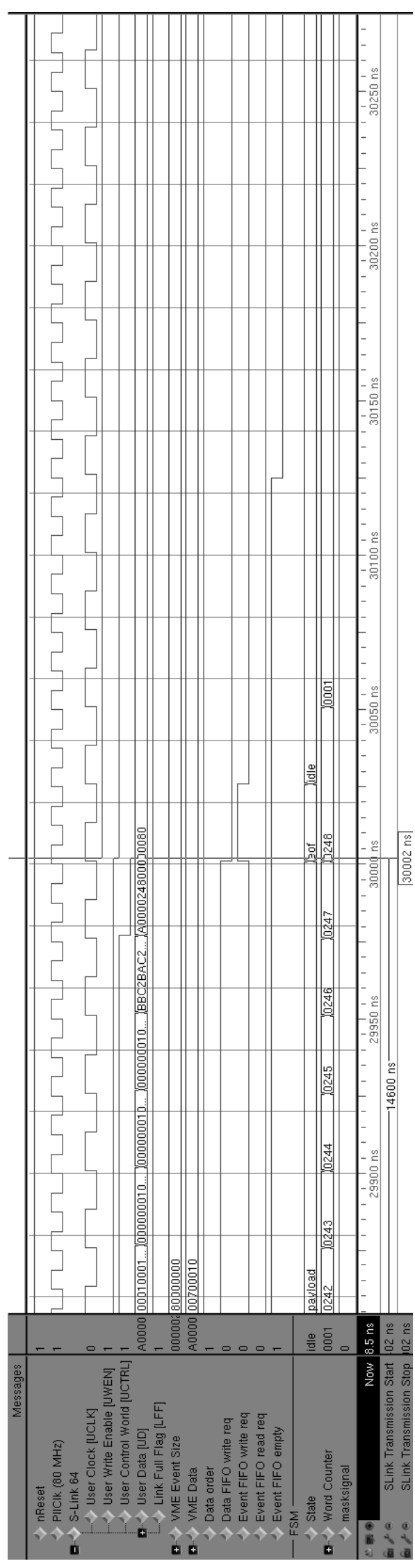

(b) End

Figure 5.11. Waveform presenting write to the OptoRx Receiver of data from the SLink interface. 


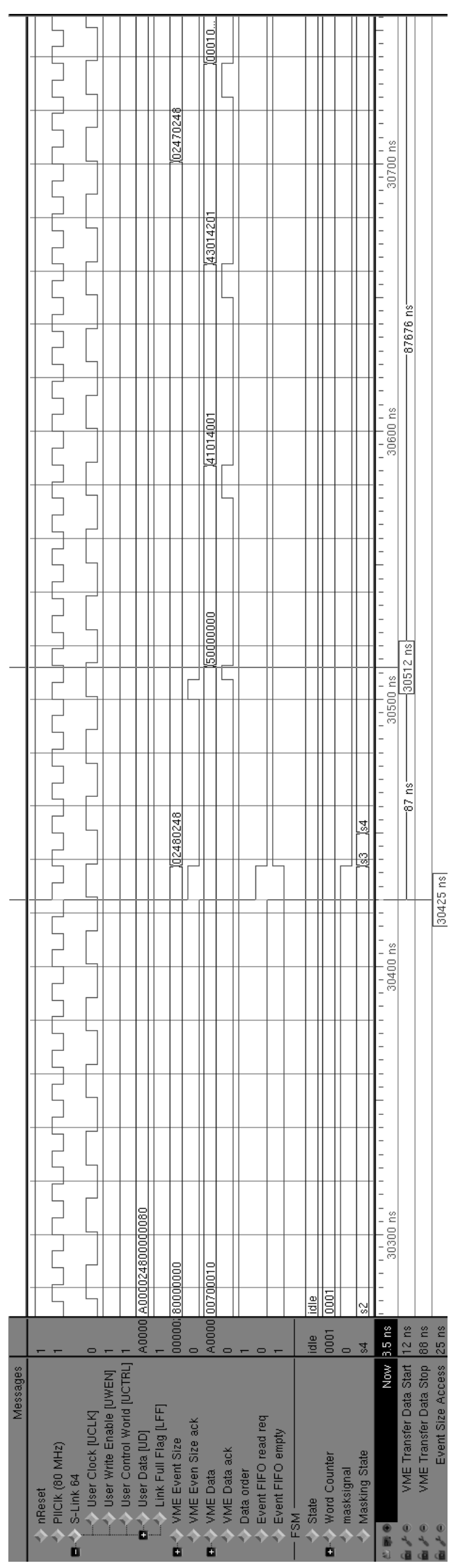

(a) Beginning

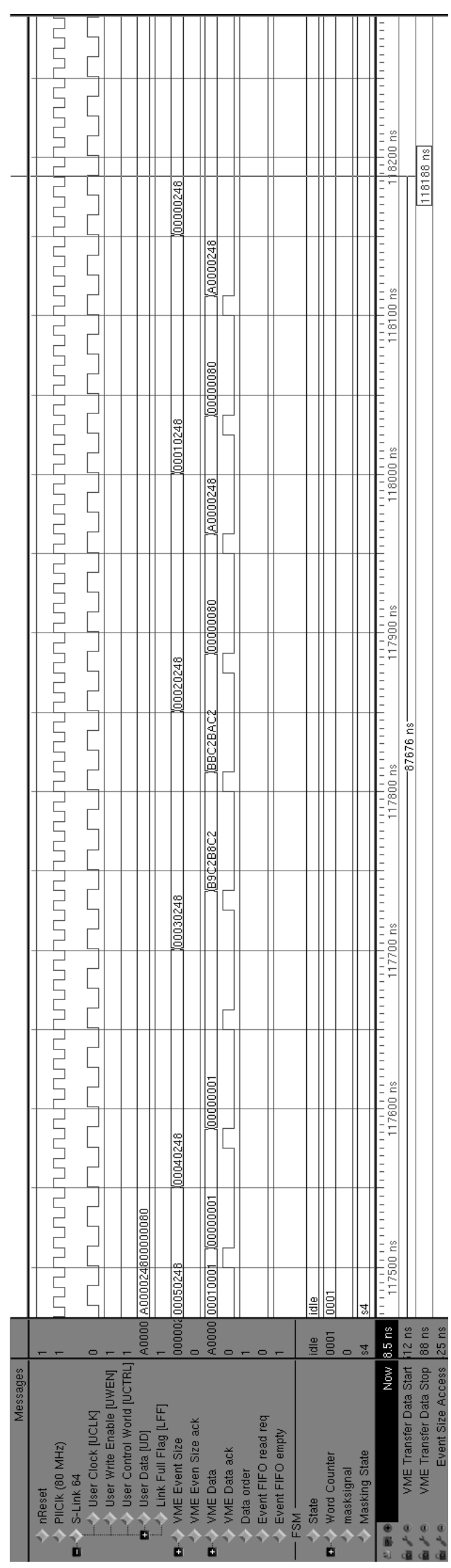

(b) End

Figure 5.12. Waveform presenting readout of the OptoRx Receiver. 


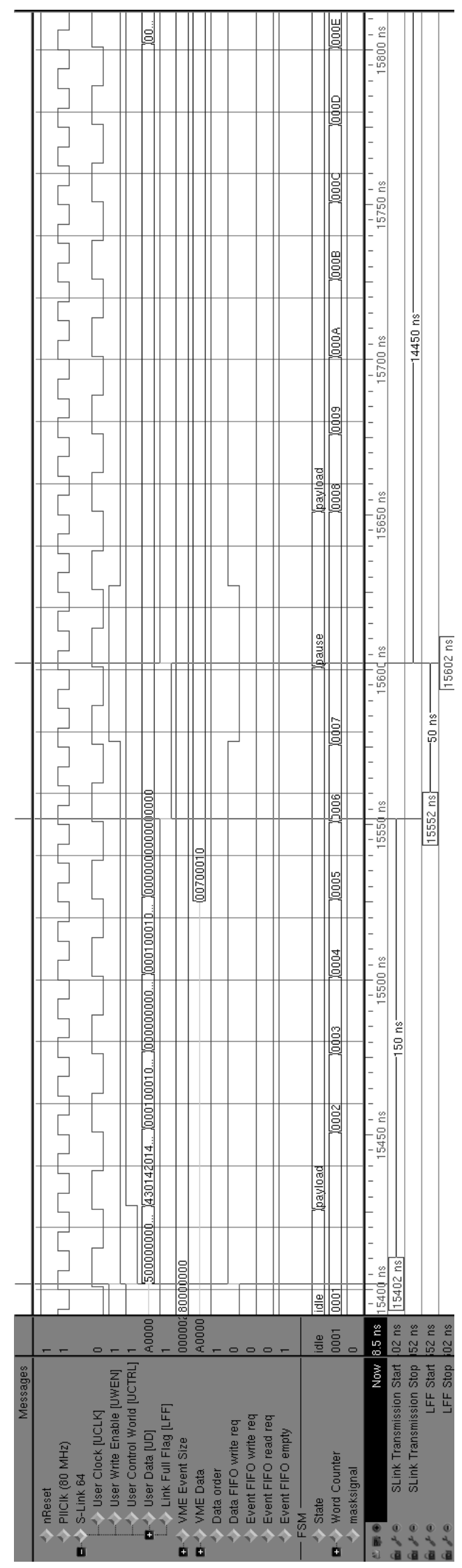

Figure 5.13. Waveform presenting write to the OptoRx Receiver excepted by the Link Full Flag condition. 


\section{The OptoRx}

\subsection{General Overview}

The OptoRx uses the most advanced FPGA in the DAQ of the TOTEM experiment: EP2SGX60EF1152C5. The device, as rest of the chips, is manufactured by the Altera and belongs to a third generation of FPGAs: the Stratix II GX family. A hallmark of the EP2SGX is a fact, that it is equipped with high-speed serial transceivers able to work with the speed up to $6.375 \mathrm{Gbps}$. The $90-\mathrm{nm}$ process node allows to supply the core with $1.2 \mathrm{~V}$. The version of the chip used in the experiment has 24176 Adaptive Logic Modules (ALMs), 2.5 Mbits of an internal memory, 8 PLLs and 12 transceiver channels. The last digit in the name customarily indicates the speed grade, which for the OptoRx is equal to $5-$ it is the slowest device in the family. Rest information can be found in the manufacturer documentation [21].

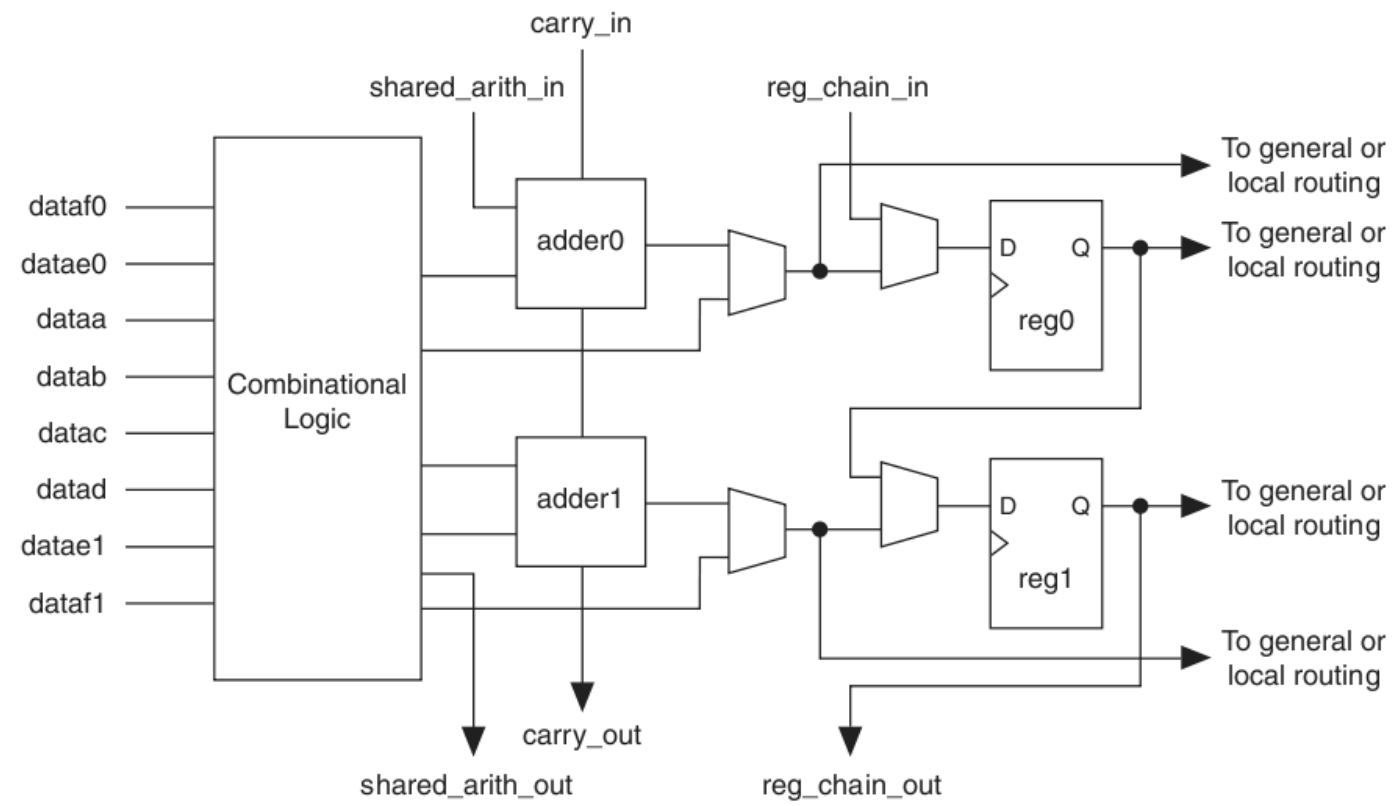

Figure 6.1. The structure of the Adaptive Logic Module (ALM) in the Stratix II Family.

The smallest logic cell in the Stratix II GX differs from the LEs occurring in the already described FPGAs. The manufacturer calls them the Adaptive Logic Modules. Figure 6.1 presents their schematid ${ }^{1}$. The combinational logic from the figure has

\footnotetext{
${ }^{1}$ copy from Altera's documentation
} 
8 inputs. It can not implement all 8-input Bool's logic function, because actually it consists of two 4-input LUTs and four 3-input LUTs. However, the ALMs still give a big flexibility, because can accomplish various combinations of two functions. This adaptability allows the ALM to be completely backward-compatible with the 4-input LUT architectures (Cyclone, Stratix). What is more, a one ALM can also implements any function of up to six inputs and certain seven-input functions. The Altera claims, that for the EP2SGX60EF1152C5, the number of the ALMs is equivalent to the 60440 LEs.

The objectives of the OproRx are:

- receive data from an optical link with speed of $800 \mathrm{Mbps}$;

- build the events according to the CMS Common Data Format (Section 2.4);

- buffer data in internal FIFOs;

- support the local S-Link interface |11];

- enable communication with the Main through the Opto-local bus;

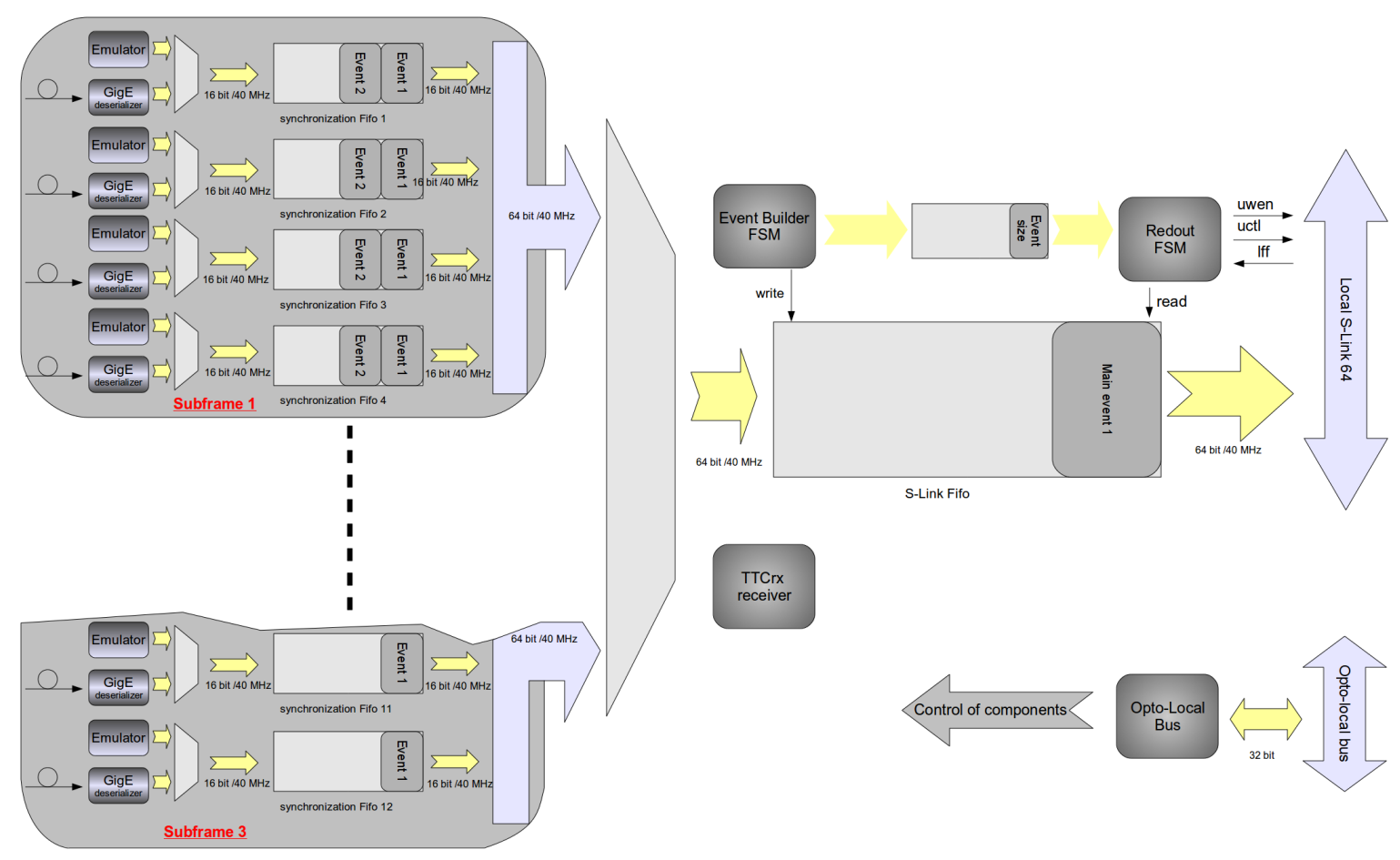

Figure 6.2. The scheme of the OptoRx.

Figure 6.2 presents scheme of the OptoRx. All parts make up on a big processing block. The implementation, thanks to emulator components, gives the opportunity to test each channel path on the TOTFed without a real data source, what is crucial, especially in the laboratory. Also being mounted in the detector, the emulators, in case of problems, allows quickly debug if it is related to the TOTFed or a other part of the experiment. The Opto-local bus controller accomplishes the communication between the OptoRx and the VME bus (throughout the Main). It is used to configure the OptoRx in a correct operational mode. The output from the processing block is routed to the Main via mentioned before, the local S-Link 64 interface. 


\subsection{Implementation}

\subsubsection{Data Emulator}

The data emulator consists of two kind of components: a one trigger emulator and sixteen VFat emulators. Figure 6.3 presents the scheme of the the emulation block.

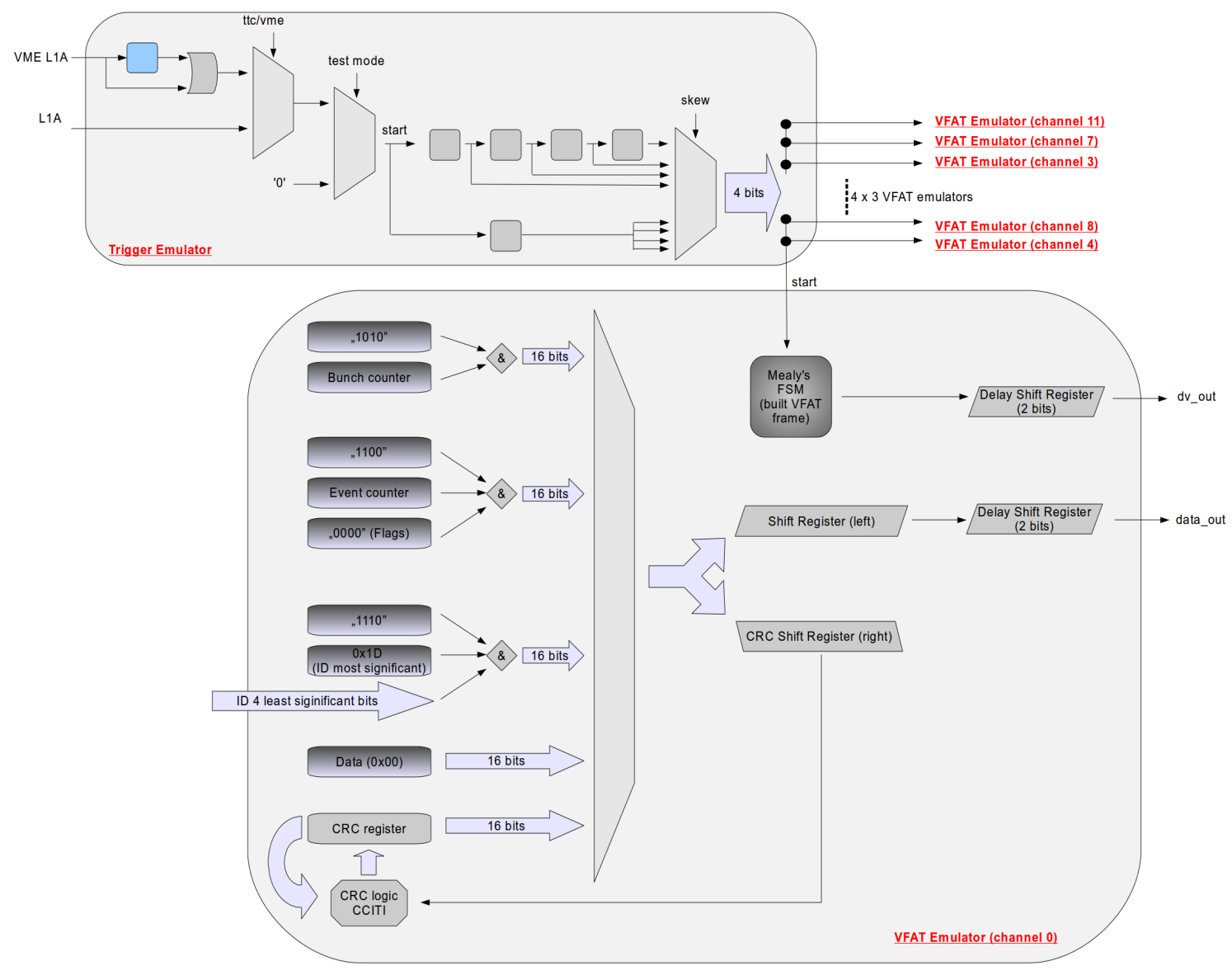

Figure 6.3. The scheme of the data emulator in the OptoRx FPGA.

\section{Trigger Emulator}

The Trigger emulator generates the signal, that starts emulation process. The entity declaration is presented below:

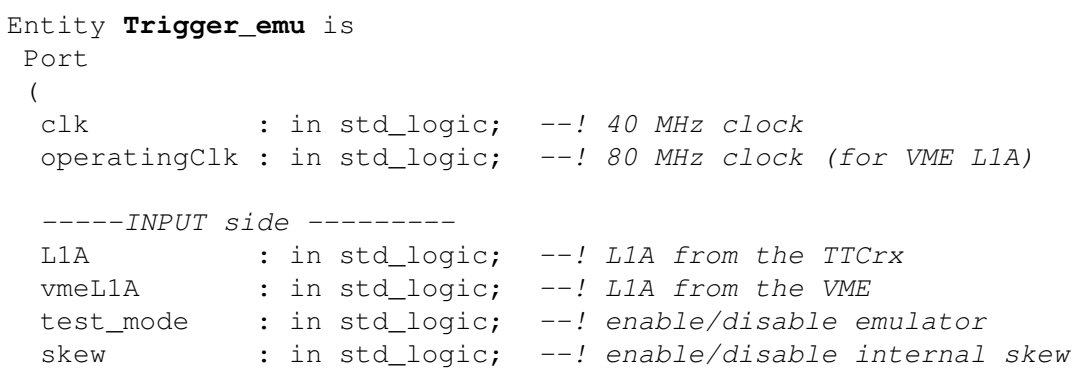




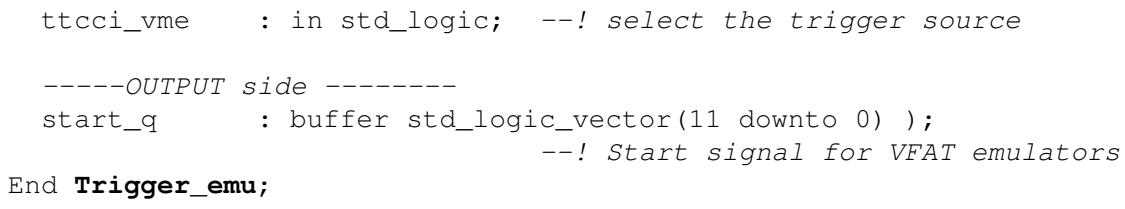

The Trigger emulator can use either the L1A signal from the TTCrx or a signal related to to register accessible from the VME bus ${ }^{2}$ (called the VME L1A in Figure 6.3), to generate vector of start strobes for the VFat emulators. In general, the component works with a processing clock (40 MHz), however usage of the VME $L 1 \mathrm{~A}$ forces to use a flip-flop clocked with $80 \mathrm{MHz}$ (marked in blue) to bridge clock domains. Selection of the trigger source is made by the ttcci_vme port.

As in the real system each GOH (Section 2.6) starts sending data independently after receiving the L1A signal, they have to be consider by the OptoRx FPGA as independent data senders having a different data arrival time. The described situation is presented in Figure 6.4.

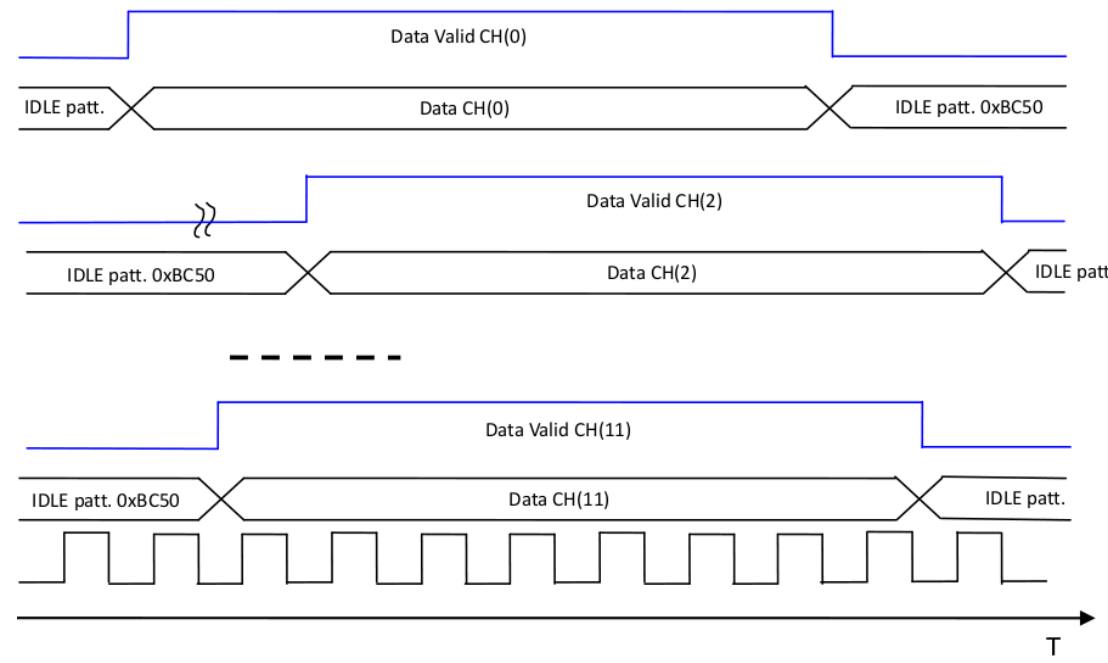

Figure 6.4. The data timing showing a different arrival time for each $\mathrm{GOH}$ in the OptoRX FPGA.

The Trigger emulator is capable to emulate the skew between each channel. The shift is fixed and changed gradually with $25 \mathrm{~ns}$ step for every group of the four subsequent channels. It means, the delay for the channel 0,4 and 8 is the same and equals to $0 \mathrm{~ns}$, for the channel 1,5 and 9 this is $25 \mathrm{~ns}$ and respectively 50 ns and $75 \mathrm{~ns}$ for the others channels. The emulation of the different arrival time can be enabled by the skew port. The test_mode enables the Trigger emulator and actually the whole emulation block, because the output start_q triggers directly the VFAT emulators.

\section{Example usage of Trigger emulator}

Figure 6.5 presents example usage of the Trigger emulator. After reset, the component is enabled in skew mode vulnerable to signals from the TTC. In $338 \mathrm{~ns}$ the L1A strobe from the specified system arrives and the entity generates vector

\footnotetext{
2 through Opto-Local and Local bus
} 
of the start signals for the Triggers emulators. In $527 \mathrm{~ns}$ following L1A is received, however the component has been disabled, so there is no action. The last test is performed for the entity sensitive to the trigger from the VME in the mode without skew. The signal arrives in $763 \mathrm{~ns}$ and the Trigger emulator correctly generates the output. One should notice, that component cooperates with two clock domains, so a pulse width of the L1A from TTC ( $25 \mathrm{~ns}$ ) is wider than from the VME (12.5 ns).

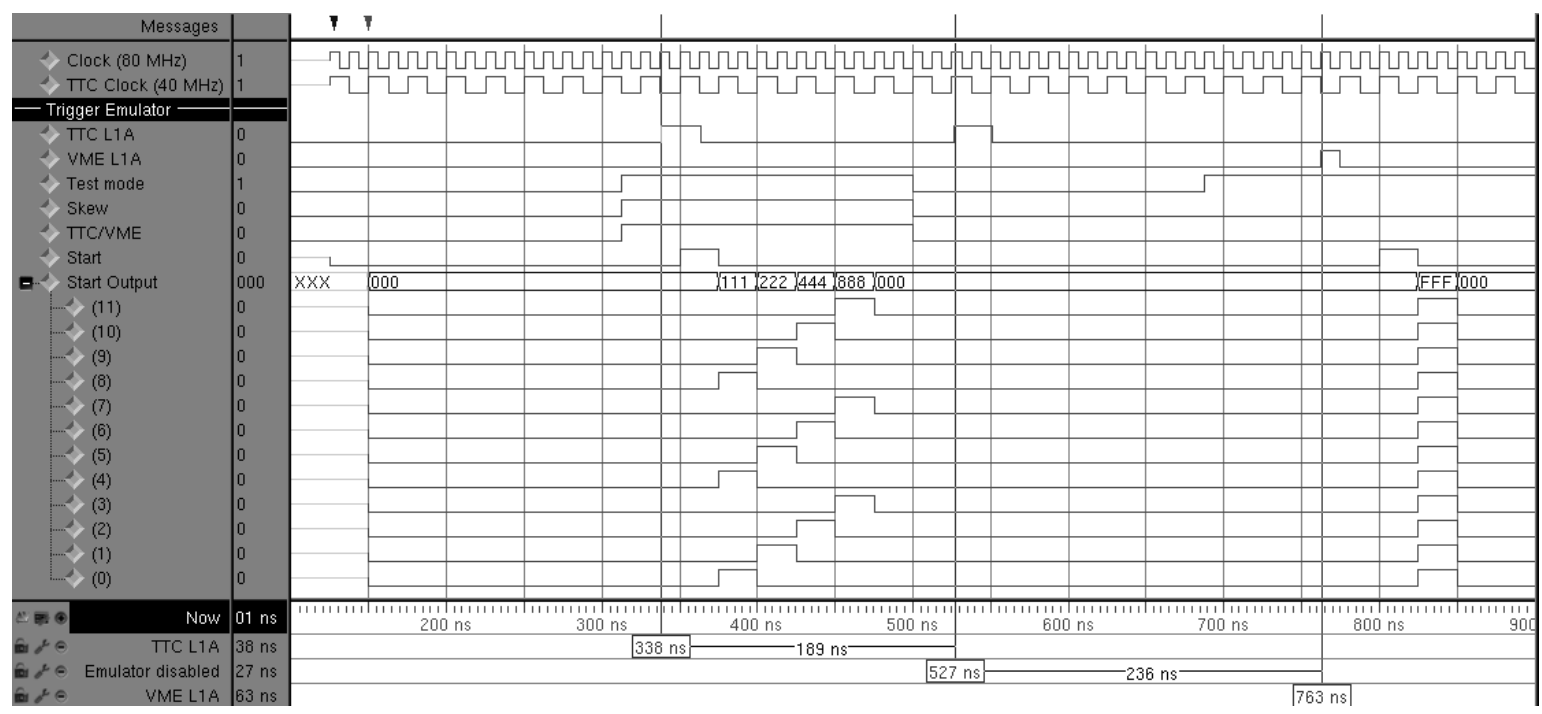

Figure 6.5. The example usage of the Trigger emulator.

\section{VFAT emulator}

The VFat emulator behaves as single GOH and prepares a valid data event frame in the CMS Common Data Format (Section 2.4) for the following processing blocks. The entity listening is presented below.

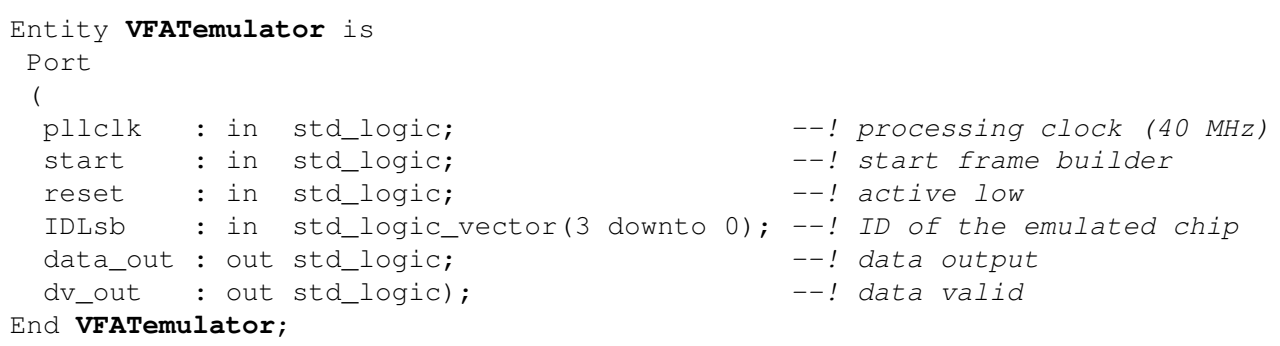

The VFat emulator is controlled by Mealy's state machine (Figure 6.6), which mainly chooses subsequent parts of the frame by a big multiplexer and generates a data valid signal. The output of the multiplexer is routed to two shift registers. One of them, which shifts data left (from MSB) is used by the data output. Second one, as it shifts data right (from LSB), is used by logic generating CRC checksum (Section 2.5). The short output shift registers compensate the delay needed by the CRC logic to calculate a current checksum. Each VFat Emulator implements the ID related to the hardware twelve bits identifier of the real VFAT chip. The eight most significant bits are fixed to the value $0 x 1 \mathrm{D}$, however four least significant bits are indicated by the IDLsb port. The 128 bits of data are stuck at 'O'. The VFat emulator is equipped with a bunch and an event counter, which assure that every 
frame is different and enable to distinguish an event loosing issue during tests by the processing part of the TOTFed.

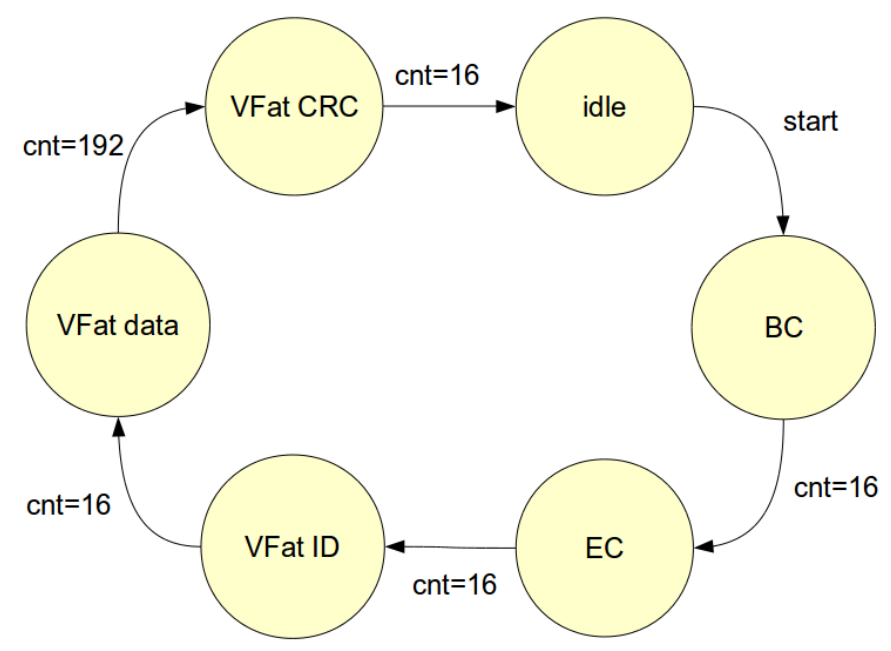

Figure 6.6. The diagram of Mealy's state machine in the VFat emulator. 'cnt' is a local counter of the FSM clocked with $40 \mathrm{MHz}$.

\section{Data Emulator}

Controlling of the emulator block is made by the EMU_MODE register (address 0x0n 180104) The meaning of each bit is presented in Table 6.1

\begin{tabular}{c|c} 
No. of Bit & Description \\
\hline $31-4$ & Not used \\
3 & Enable the emulator mode (0 - normal mode) \\
2 & $\begin{array}{c}\text { OptoRx mode (0 - DAQ mode, processing block is used) } \\
\text { (1 - Trigger mode - raw data routed to the Main) } \\
1\end{array}$ \\
0 & Enable skew between frames \\
& Source of the trigger: TTCrx $(1)$ or VME(0)
\end{tabular}

Table 6.1. Description of the Emulation Mode register.

The data emulator is able to emulate the 12 fully consistent VFAT frames coming from the 12 separate GOHs. The 12 dummy chip IDs stretch from 0x1D0 to Ox1DB. It means, that there is only a one dummy VFAT frame, out of 16 , for each GOH fiber. The frame is generated on the first position over 16 inputs available on each channel. The output data format after processing in emulation mode is presented in Figure 6.7

\section{Example usage of the VFat emulator}

Figure 6.8 presents behaviour of the VFat emulator. A trigger arrives in $375 \mathrm{~ns}$. The FSM goes to the $B C$ state and selects the header of the VFat frame at the output of the major multiplexer from Figure 6.3. Once load strobe is asserted, the value is written to the Shift register and the CRC Shift register. Data propagate through Delay shift registers and finally, after three clock cycles since they have

\footnotetext{
${ }^{3}$ where 'n' corresponds to the number of the OptoRx
} 


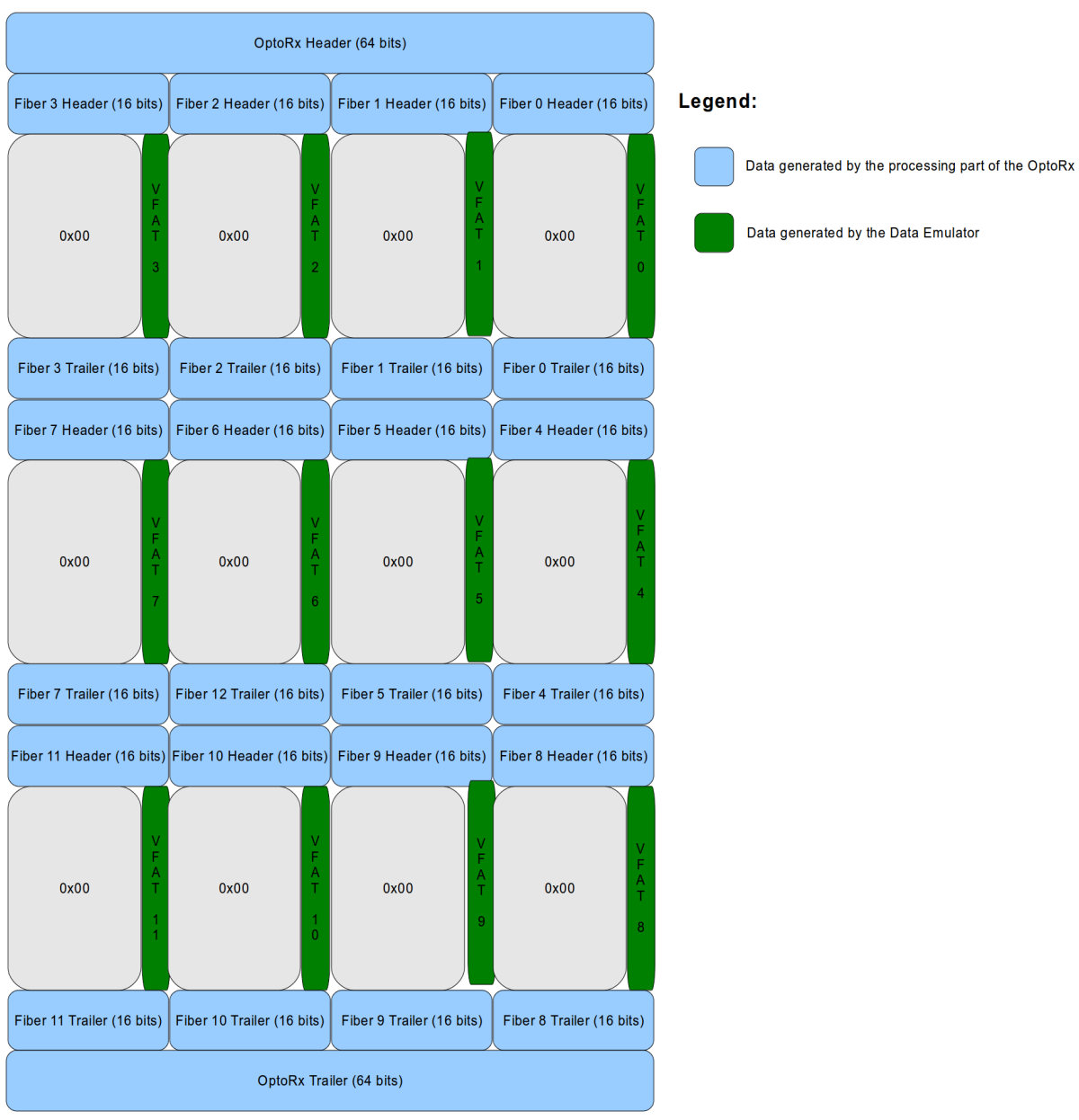

Figure 6.7. The data structure after processing at the output of the OptoRx FPGA in the emulator mode.

appeared on the output of the multiplexer 4 , they reach the external port (475 ns). This situation is indicated by assertion of the Data valid strobe. As data are sent serially, the state machine uses internal counter to switch among the following states. The CRC input signal is related to the input of the CRC logic CCITI from the scheme.

In the VFatCRC state, as the CRC block has to use data from the port, whose value is delayed about 3 clock cycle to the multiplexer input, once the counter of the FSM equals to 2, the Load MSB of CRC is asserted and the two most significant bits of the CRC checksum are written parallel to the Delay shift registers. It allows to send the VFat frame in a one continuous part.

The waveform in Figure 6.8 presents that one VFat frame lasts 4800 ns and its preparation takes $4900 \mathrm{~ns}$. What is more, the working VFat emulator is insensitive to incoming triggers. For this reason the maximum frequency of the L1A for the OptoRx in the emulator mode is $200 \mathrm{kHz}$.

\footnotetext{
${ }^{4}$ One clock cycle through the shift register and 2 through the delay shift registers
} 


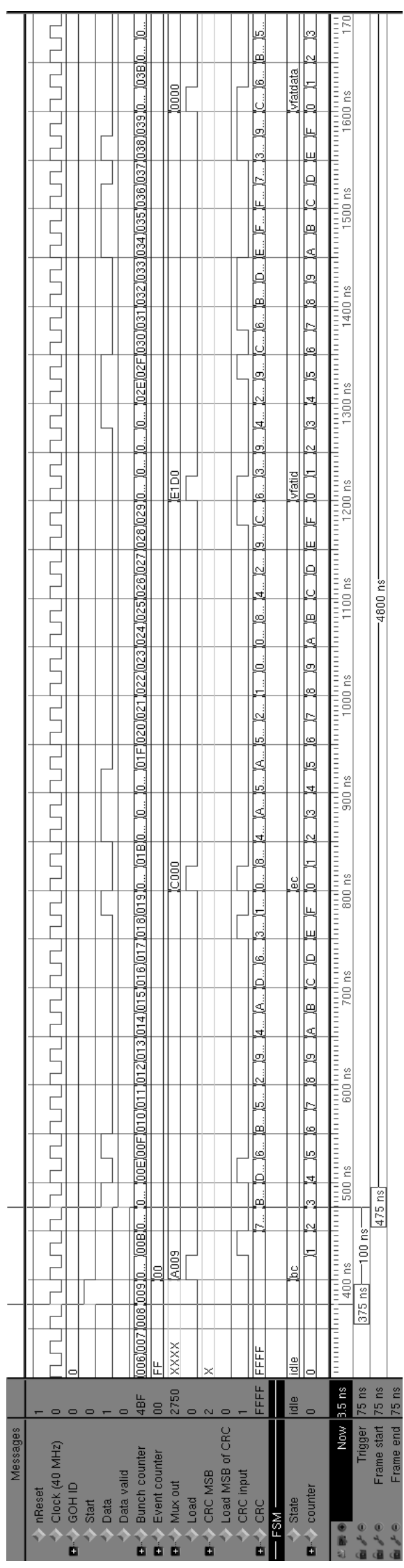

(a) Beginning

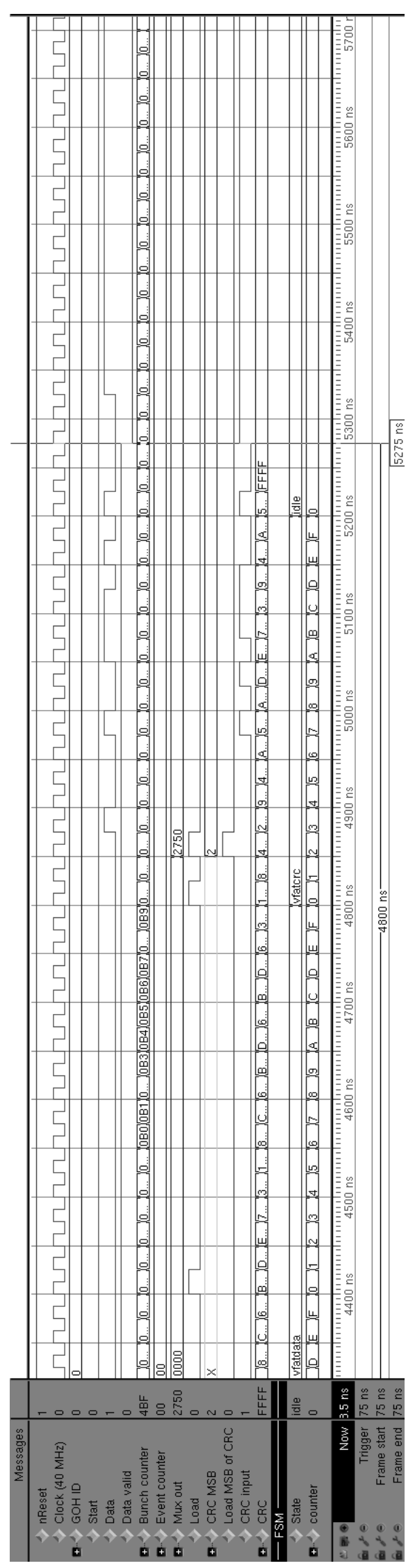

(b) End

Figure 6.8. Waveform presenting work of the VFat emulator. 


\subsubsection{Opto-local bus}

The Opto-local bus provides access to all registers of the OptoRx FPGA from VME bus ${ }^{5}$. They are used only to configure the device and read its status. Data coming from the detectors (VFats) are sent by the dedicate local S-link interface connecting the OptoRx with the Main.

The Opto-local bus implementation uses the same generic VHDL code as the Local bus slave controller from the Main FPGA (Section 5.2.1. However, there are some differences:

- the address width is 8 bit.

- the data width is 16 bit.

- as Opto-local bus connects always two device (a master - the Main and a slave - the OptoRx) the slave strobe is no longer a tri-state line.

- the OptoRx is a last FPGA in a chain of buses, so there is no a crossbar multiplexer switching, according to the current address, buses connected to the output.

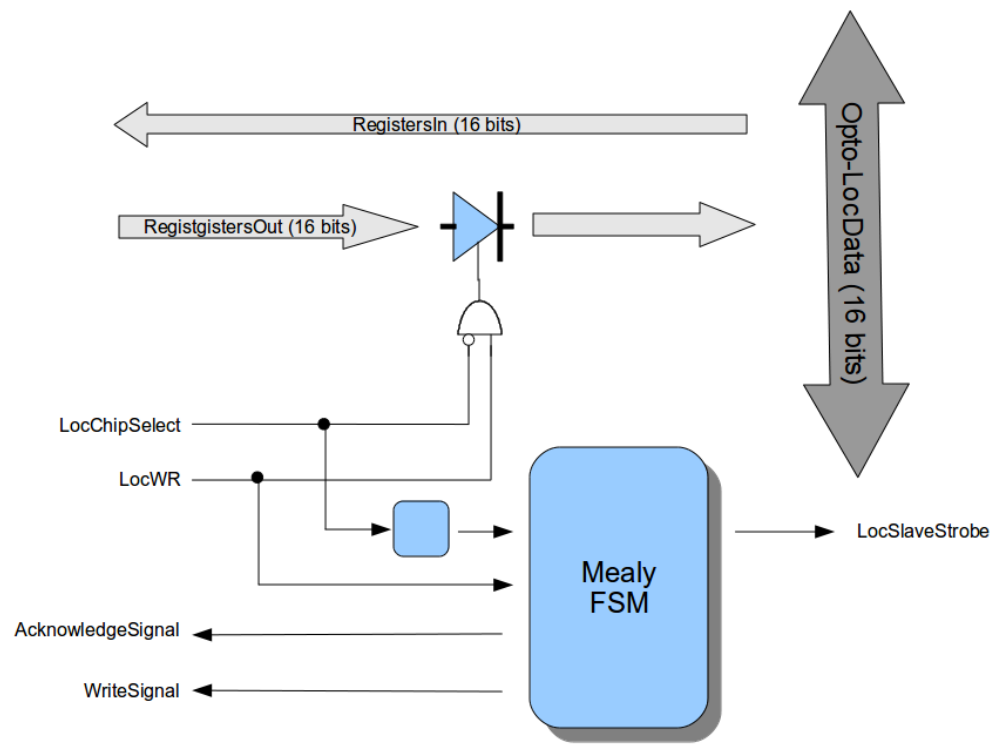

Figure 6.9. The scheme of the Opto-local bus slave controller.

Figure 6.9 shows the scheme of the Opto-Local bus slave controller. As it does not have a bus crossbar, it is much simpler than the Local bus version. The component is managed by a fast Mealy state machine clocked with a $80 \mathrm{MHz}$ clock.Because the interface between the OptoRx and the Main is asynchronous as the Local bus, to avoid hazards, the crucial signal - Local Chip Select is latched by an additional flip-flop.

The FSM has less states, than the implementation from the Main (Figure 6.10). At the beginning it expects the chip select signal ${ }^{6}$. Depending on the LocWR signal, if it is low, the state machine asserts the write request for the internal registers and goes to the writing state where it waits until the master deny the chip select. Otherwise, the FSM switches to the reading state. As soon as the Main FPGA deny

\footnotetext{
5 throughout the Local bus

6 active low
} 


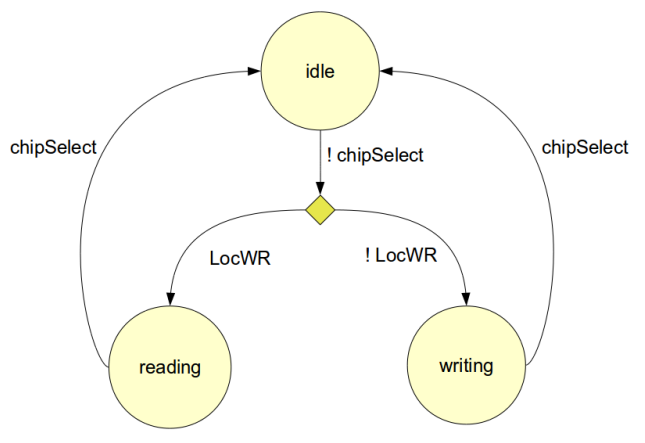

Figure 6.10. The state transitions of the Opto-local bus slave controller.

the chip select, it asserts the read acknowledge signal and on a nearest rising clock edge goes back to the idle state.

A structure of a multiplexer tree, which splits signals from the Opto-local slave controller to internal components, depends on the generic parameter $\mathbf{T}$. If it is 'DAQ_BOARD' the multiplexer tree is the same like in the Main $7^{7}$ (Figure 6.11). However, for T equals to the 'TRIGGER_BOARD', the tree contains additional registers specific for the Trigger TOTFed. This approach allows to use the same VHDL code in two kind of the motherboard.

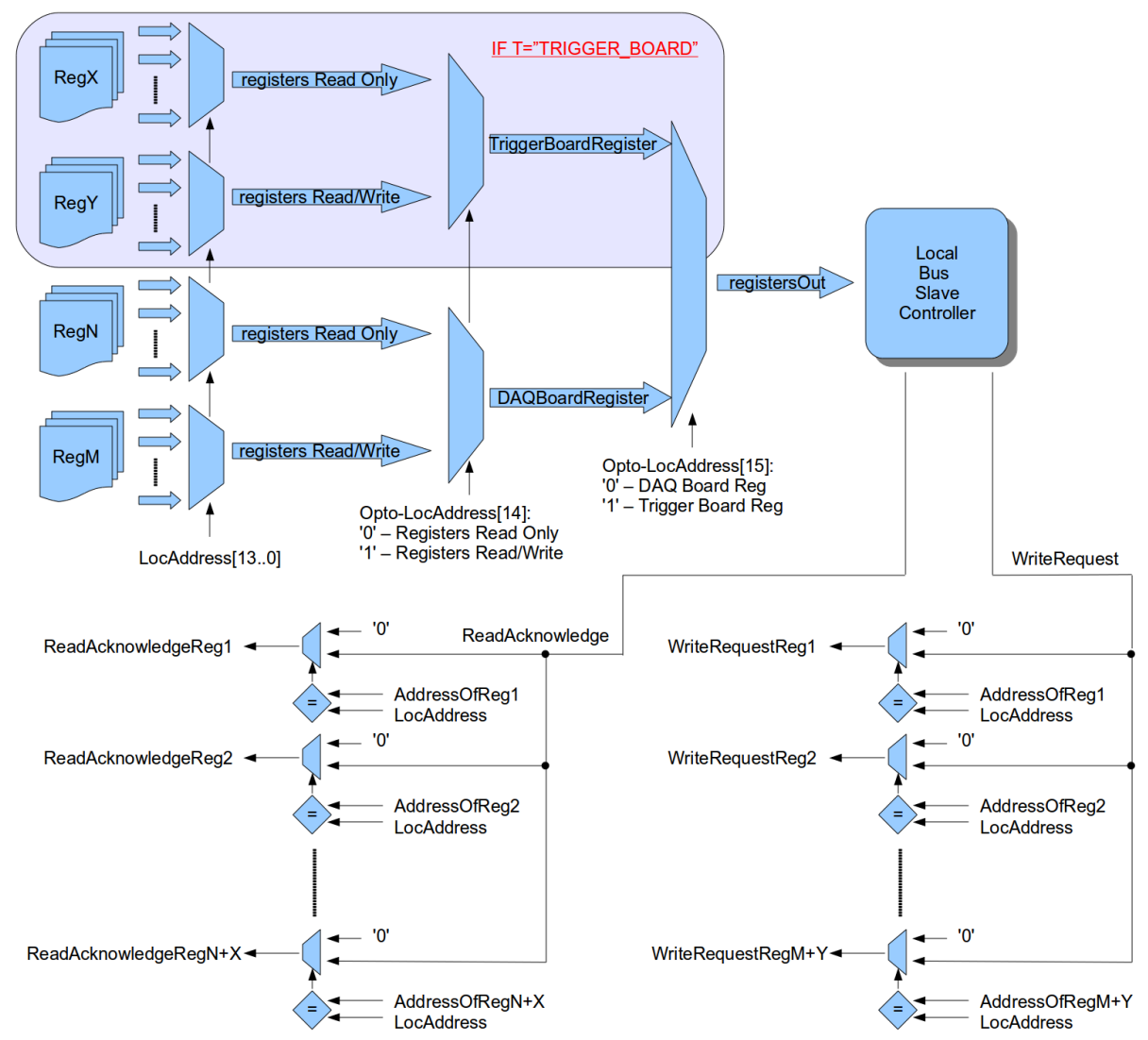

Figure 6.11. The multiplexer tree from the OptoRx.

\footnotetext{
${ }^{7}$ of course the multiplexers use different bits of the address
} 


\section{Example usage}

Figure 6.12 presents the working Opto-local bus slave controller. The access is made to the Debug Counter of the OptoRx 1. The value which is written during a first access (210 ns) is read at the end of simulation (296 ns). One should notice the fact, that both transfers take the same amount of time.

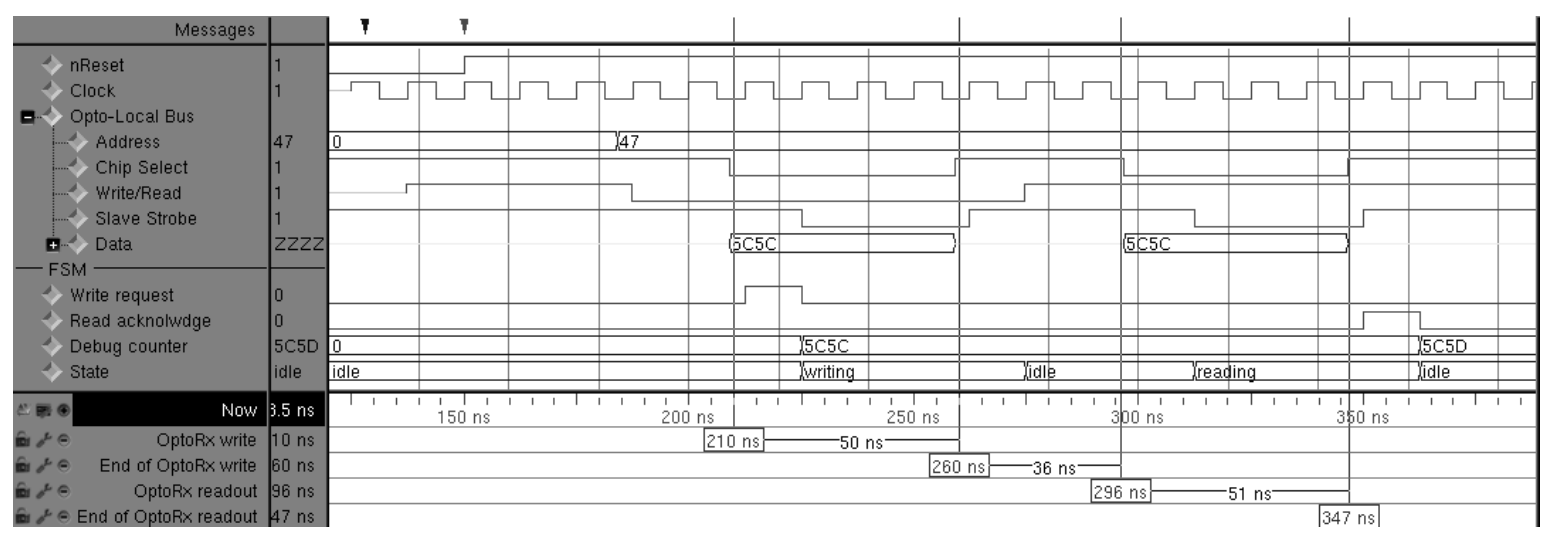

Figure 6.12. The waveforms presenting usage of the Opto-local bus slave controller from the OptoRx.

\subsubsection{TTCrx Receiver}

The TTCrx Receiver is used by the processing part to obtain an information from the TTC system (Section 2.3.3) about timing of the LHC machine. The time stamp of each event is specified by a value of two counters: Bunch Crossing Counter and Event Counter. The OptoRx internally calculates them and compares with the results received from TTCrx chip via the TTCrx_receiver_4bit entity. This approach allows to the OptoRx FPGA specify a TTS state 8 and alert the DAQ in case of missing events. The declaration of the TTCrx_receiver_4bit is presented below:

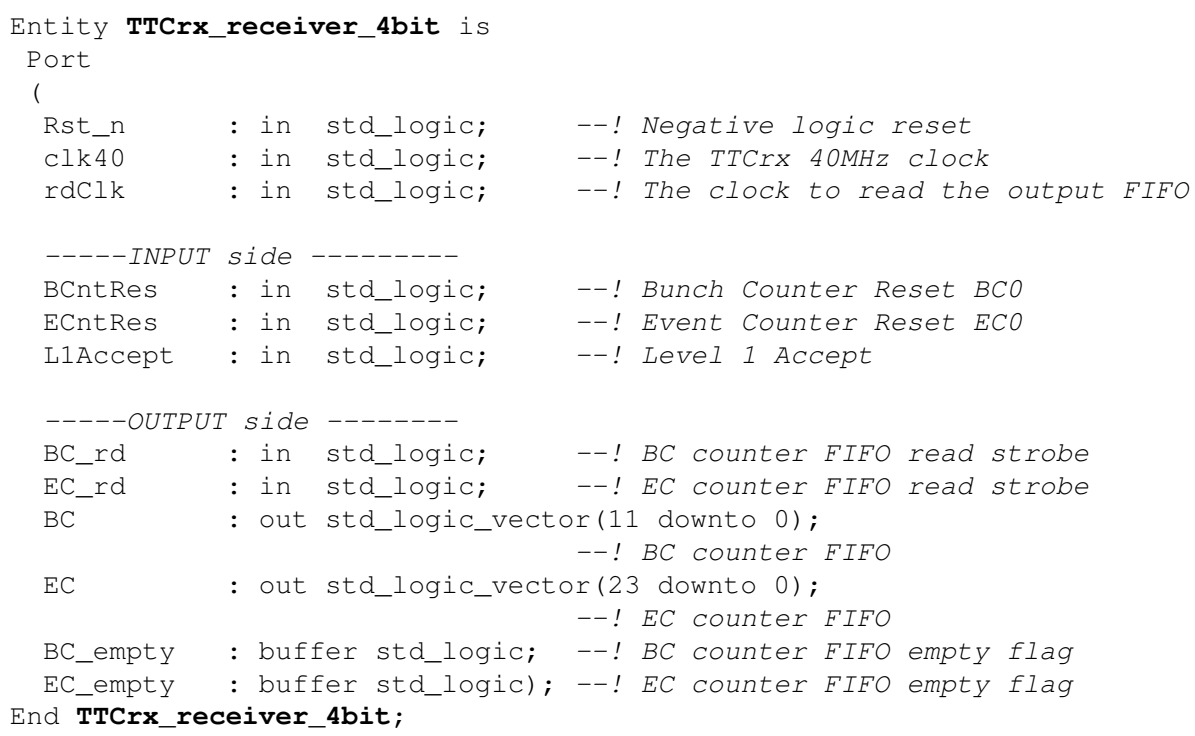

${ }^{8}$ for example the out of synch 


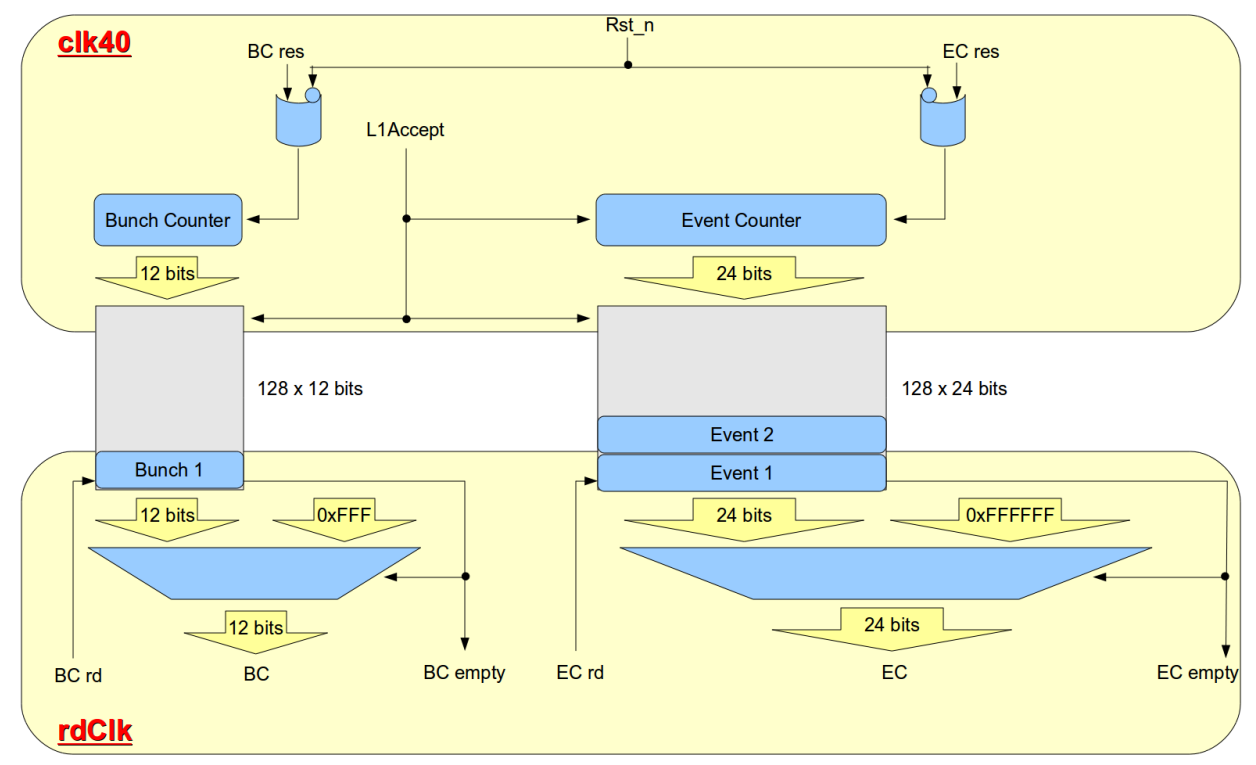

Figure 6.13. The scheme of the TTCrx Receiver.

Figure 6.13 presents the scheme of the TTCrx Receiver. The module bridges two clock domains. The counters and the write ports of FIFOs use the raw $40 \mathrm{MHz}$ clock from the TTCrx chip. The readout part of the component uses $40 \mathrm{MHz}$ clock from a PLL of the FPGA, which is shared by the entire processing block. The Bunch counter increases its value with every clk 40 cycle. The Event counter is gated by the L1Accept strobe. Both of them are synchronized to the TTC system by respectively the BCntRes and the ECntRes signals witch reset their values?

The FIFOs used in the entity have two objectives: bridge the two different clock domains and store the following time stamps 10 . The depth of the Bunch Fifo is $128 \times 12$ bit, whilst Event Fifo is $128 \times 24$ bits. As the two queues, to obtain an instant answer, use show-ahead implementation and embedded memory in the FPGA can not be reset, their outputs are multiplexed between fixed patterns ${ }^{11}$ and current data. The recipient to read a next word should assert appropriate read acknowledge strobe.

\section{Example usage}

Figure 6.14 presents example usage of the TTCrxReceiver. In $250 \mathrm{~ns}$ the LHC machine synchronizes the counters asserting for $25 \mathrm{~ns}$ the bunch and event counter reset. Since $340 \mathrm{~ns}$ the modules has been received three subsequent L1A signal from trigger source. The Event builder FSM (Section 6.2.5) reads both counter values simultaneously (700 ns) to code a TTS status. During real work, following data readouts made by data processing have much lower frequency ${ }^{12}$ - the figure

\footnotetext{
${ }^{9}$ The signal is generated by the TTC when the global, LHC counter is overflowed.

10 so the OptoRx can still processes a first event, even if new have arrived because of the burst trigger

11 when FIFOs are empty

12 the implementation limit for the readout period is equal to $14600 \mathrm{~ns}$ - the time needed to build a one event frame
} 
shows results of a synthetic testbench. The waveform justifies the usage of the multiplexers assigned to the show-ahead FIFO, which mask its outputs 13 .

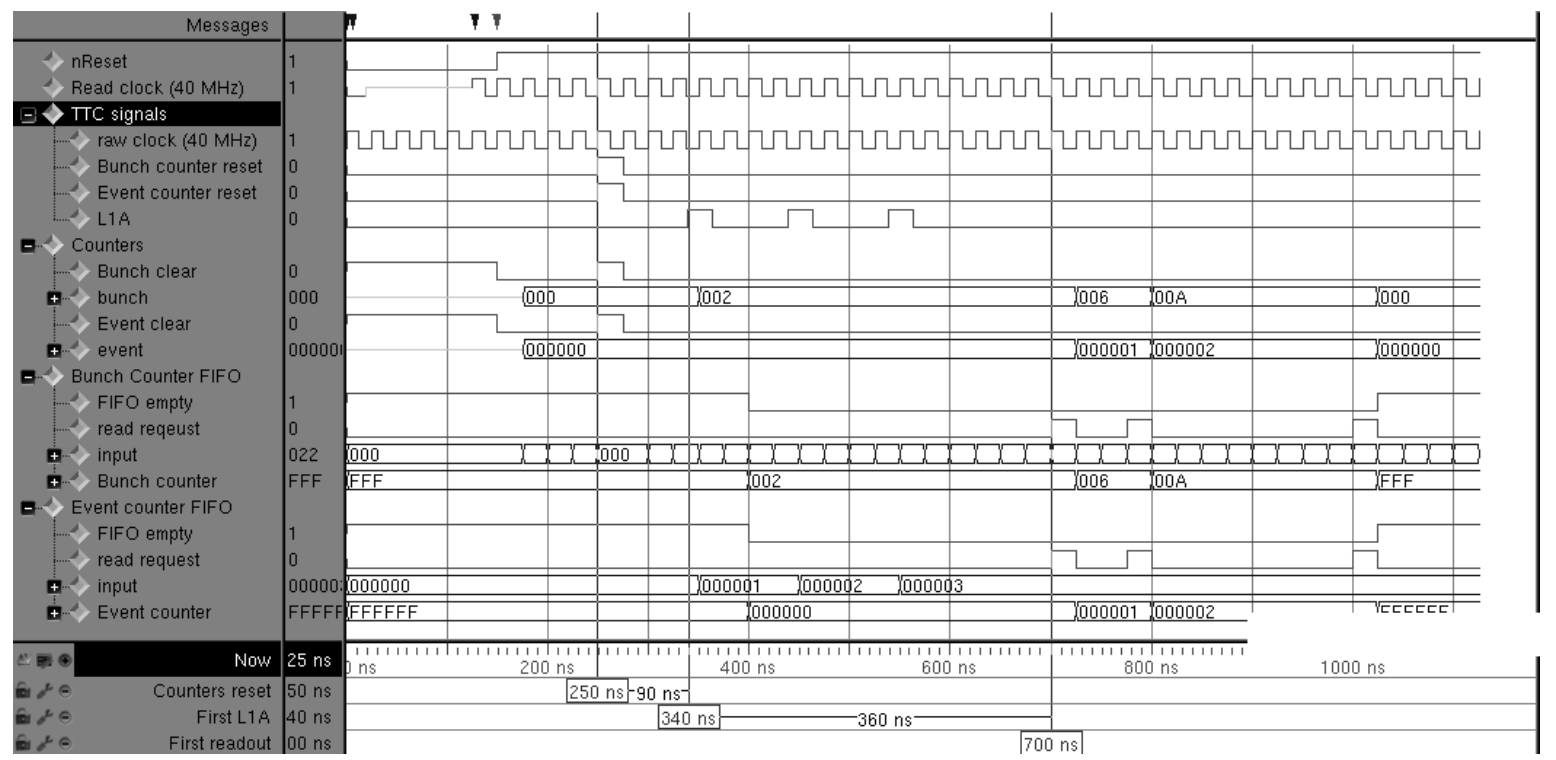

Figure 6.14. The waveform presenting usage of the TTCrx Receiver.

\subsubsection{Channel data processor}

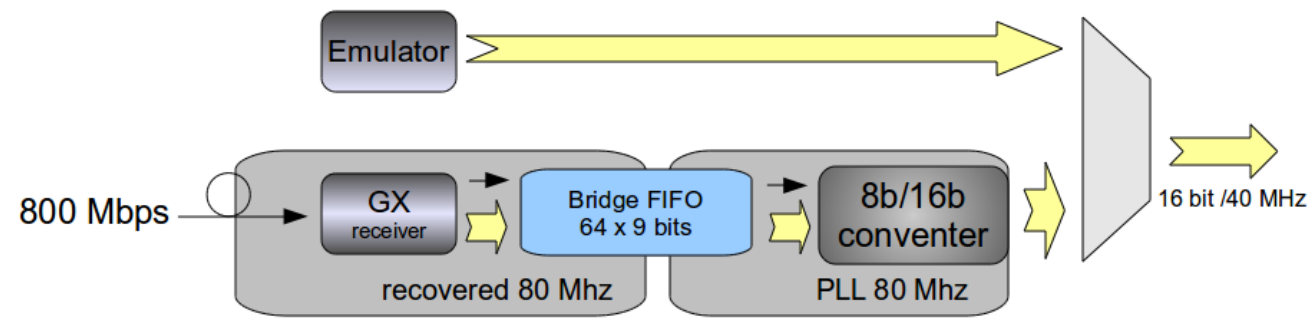

\section{Channel data processor}

Figure 6.15. Scheme of the Channel data processor.

The Channel data processor is a device, placed between the fiber and the synchronization FIFO (Section 6.2.5) selecting data source for the data processing. Its general scheme is presented in Figure 6.15.

Rate of incoming data from the $\mathrm{GOH}$ (Section 2.6) is equal to $800 \mathrm{Mbps}$. The Stratix GX transceiver is set in receiver only mode. The Altera's megawizard allows to treat all twelve channels together, what simplify configuration. The receiver exploits the $80 \mathrm{MHz}$ clock generated by a PLL of the FPGA as a reference to recover the clock signal of the GOL. It uses the basic protocol with the $8 \mathrm{~b} / 10$ coding [21] and the 8-bit output. As the serial speed is quite low for a such kind of devices,

\footnotetext{
13 they are unspecified at the beginning
} 
the internal deserializer operates in single block width. The world aligner of the transceiver is sensitive to the $\mathrm{K} 28.5$ - code $(\mathrm{OxBC})$. The flag indicating the control world is written as a MSB in the bridge FIFO. The queue is able to store 64 words of 9 bits. Its write port uses the recovered clock from the receiver and the synchronization flag as a write strobe.

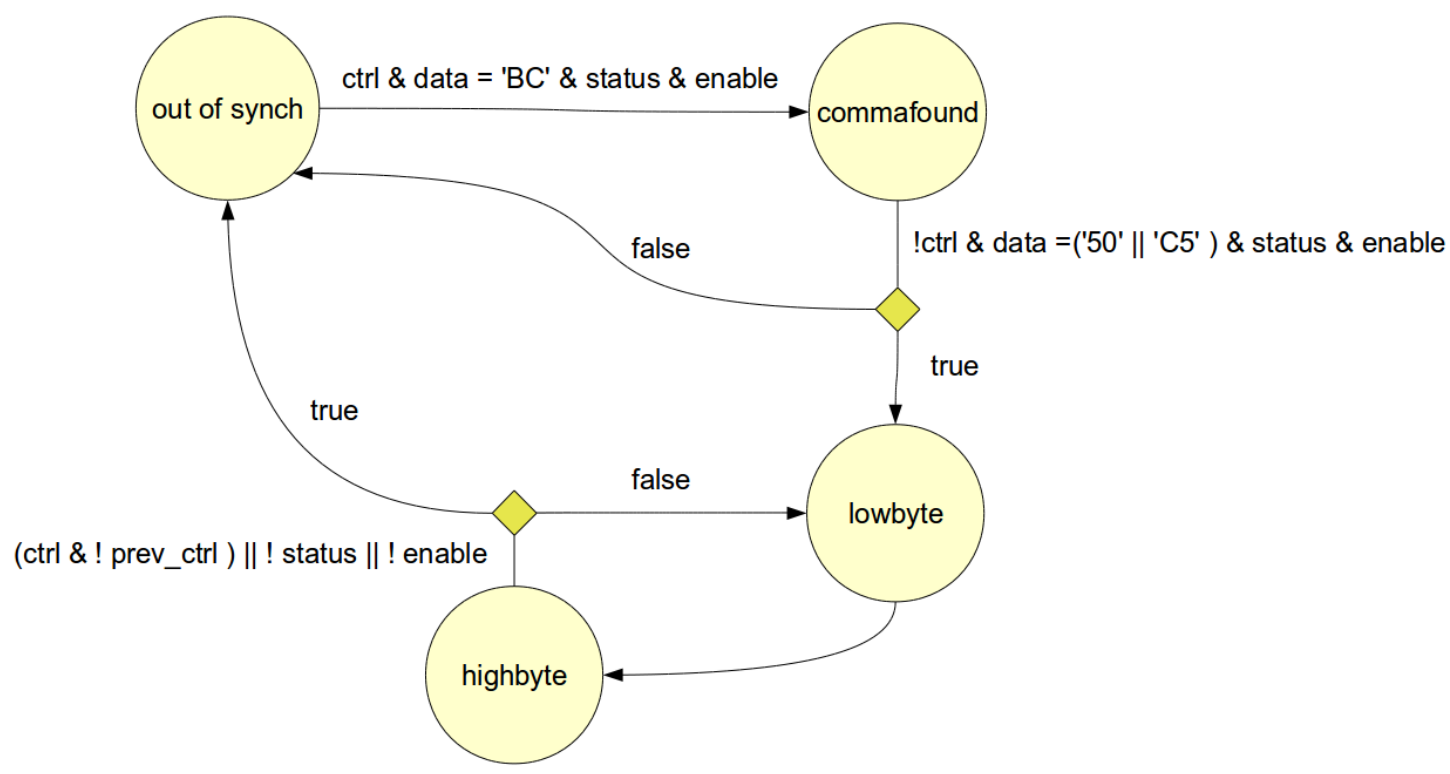

Figure 6.16. State transitions of the $8 b / 16$ converter.

The $8 b / 16 b$ converter is the Moore state machine which prepares aligned data words for the processing block. It it clocked with the $80 \mathrm{MHz}$ from the PLL and internally bridges the $40 \mathrm{MHz}$ and $80 \mathrm{MHz}$ clock domains. In the out of synch state, the FSM reads following data from FIFO expecting the GOH comma pattern $(\mathrm{OxBC})$ (Section 2.6. If in addition the control word flag ${ }^{14}$ is asserted, transceiver is enabled and has got the synchronization to the data source, the state machine goes to the commafound. Once a next word corresponds to the the LSByte of the GOH pattern (0x50 or 0xC5), the FSM moves the lowbyte state. Otherwise it backs to the out of synch. In the lowByte the LSByte of $\mathrm{GOH}$ frame is buffered in an internal register and the FSM goes to the highByte. Here it issues the full 16-bit word and asserts the Data Valid strobe for the following components on the data path. Both signals are written to an additional register. As long as the receiver keeps the synchronization and the comma pattern is not sent by the GOH, the state machine toggles between the lowByte and the highByte. Otherwise it comes to the out of synch.

14 the MSB of the FIFO output 


\subsubsection{Data Processing}

The declaration of the processing entity is presented below:

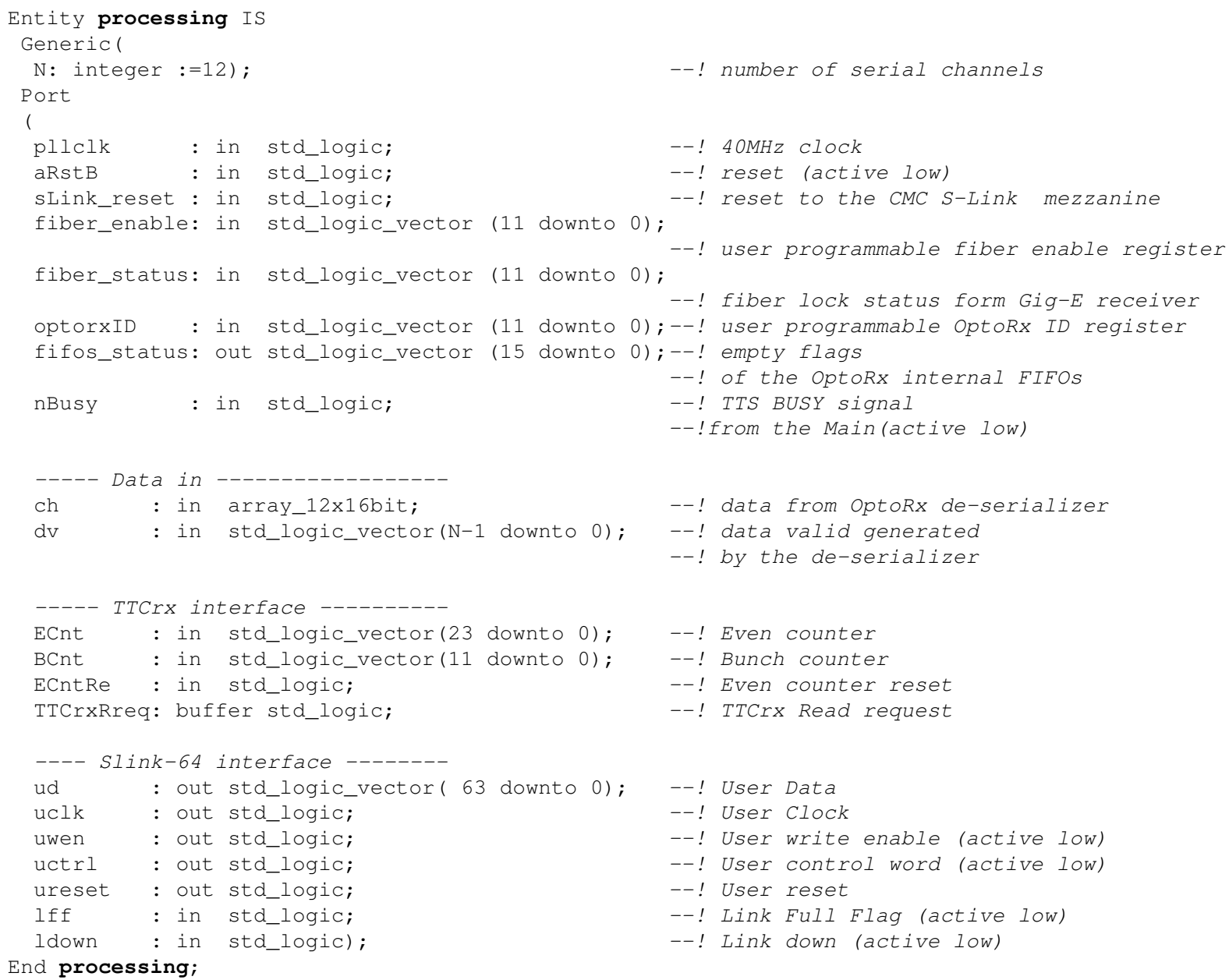

The processing component consists of two blocks (Figure 6.17). One of them accomplishes the synchronization of incoming data. Second one enables building of the S-Link packet according to the CMS Common Data Format (Section 2.4). Each entity is clocked with the same frequency as the LHC machine $-40 \mathrm{MHz}$. 


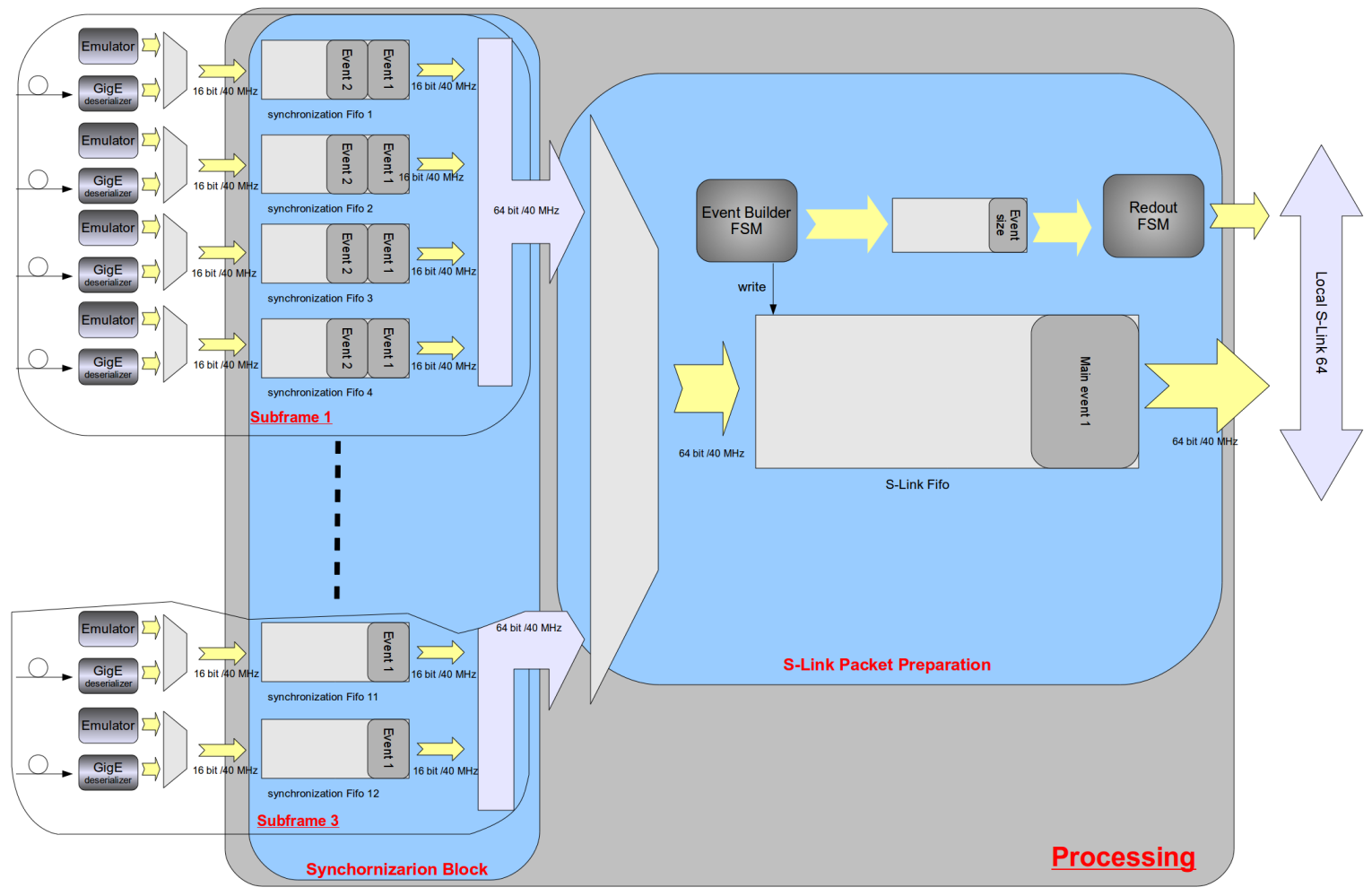

Figure 6.17. The Data processing consisting of two blocks.

\section{Synchronization block}

The synchronization block is a vector of 12 basic cells grouped in four. Each group is called subframe and contains data from four GOHes 15 ,

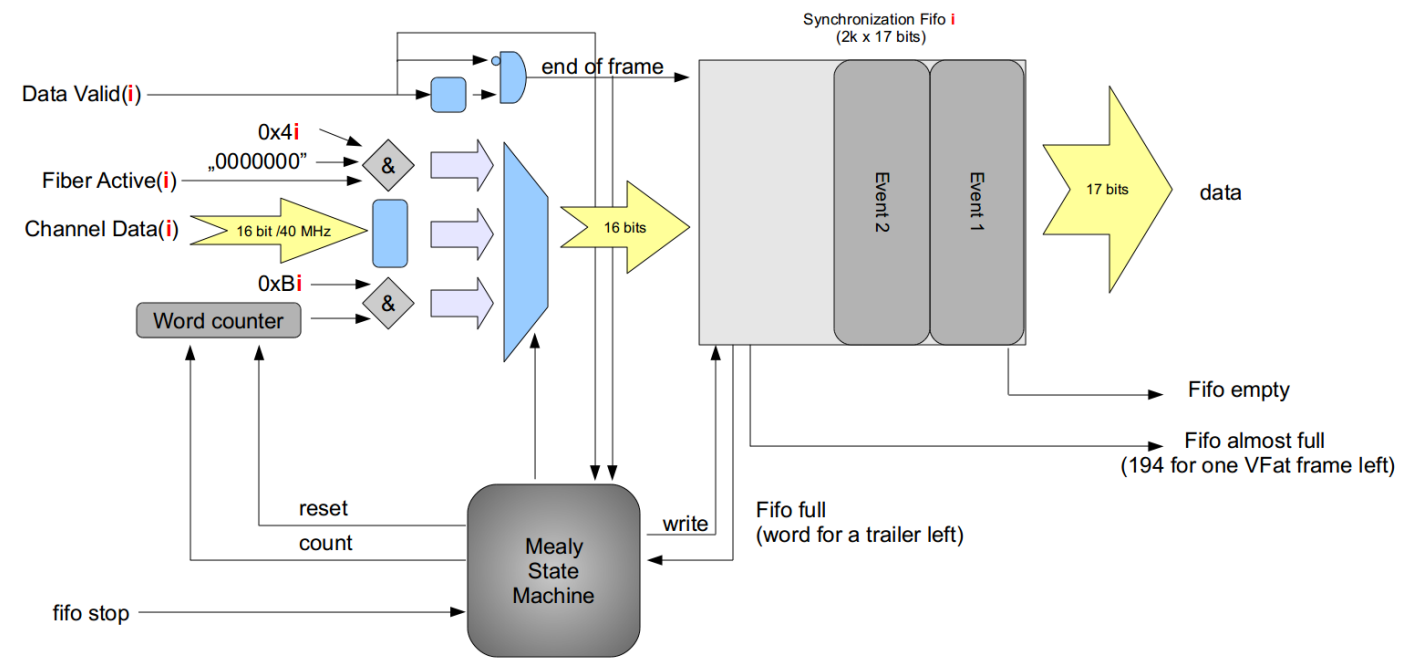

Figure 6.18. Basic cell of the synchronization block in the processing entity.

\footnotetext{
15 in normal mode
} 
The coming data are buffered in the synchronization FIFO. Its depth is $2 \mathrm{k}$ of 17 bits words. The input multiplexer selects a data source for the built-in memory between: a header, a data payload and a trailer. The data from GX receivers are registered. This approach cuts the data paths and allows the fitter to find an optimum placement for a logic close to the FIFO, which is no longer hitched to the GX transceiver. The primitives ${ }^{16}$ on the top of the Figure 6.18 generate from the original and shifted data valid signal a single strobe indicating the end of the current frame. This information is used not only by the state machine from the figure, but also, as it is stored as MSB in the synchronization FIFO, by following block in the processing entity. Additionally, the basic cell provides the Fifo almost full flag, which is high, when available space for the write port of the synchronization FIFO is less than 194 words ${ }^{17}$.

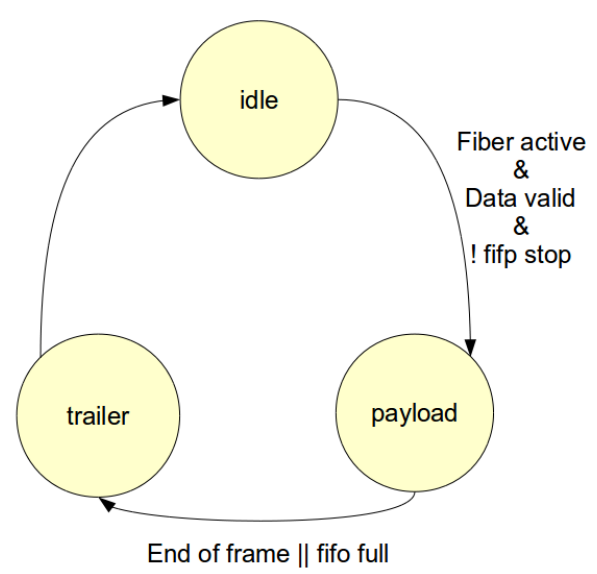

Figure 6.19. The state transitions of the FSM from the basic synchronization cell in the processing entity.

The basic synchronization cell is controlled by the Mealy state machine. Its state transitions are presented in Figure 6.19. At the beginning, in the idle state, the FSM checks the data valid, the fiber active and the fifo stop signals. The fiber active is a result of logical 'and' function between fiber enable, the flag set by user to enable a specific channel in the data acquisition, and the fiber status which indicates if the GX receiver is synchronized to the GOH data source. The fifo stop is asserted if any of the synchronization basic cells has space for less than 194 words 18 . This allows to pretend the system from acquisition of incomplete events. If the data valid is asserted, the fiber is active and all synchronization cells are able to collect one more GOH frame, the FSM writes the header to the FIFO and goes to the payload state. Here it buffers following words of data, expecting the end of frame or thesynchronization Fifo ful 19 flag. In both cases it goes to the trailer state, writes appropriate data and eventually goes back to the idle.

\footnotetext{
16 flip-flop and 'and' gate

17 equivalent of the one VFAT frame with the header and the trailer

18 logical 'or' function of the Fifo almost full flags from each basic synchronization cell

19 asserted when one word for a trailer left
} 


\section{Example usage of the synchronization cell}

Figure 6.20 presents the synchronization cell of channel 0 receiving data from the data emulator. In 475 ns the component receives the Data valid. The channel data are delayed about one clock cycle to gain a time need by the synchronization FIFO to store the header (0x4001). After this, the FSM moves to the payload state and count the following data words. When transmission of the VFat frame (5 $275 \mathrm{~ns}$ ) is over, the state machine writes to the FIFO the trailer (0xBOC2) combined with the end of frame flag as MSB. The Channel O LSB signal presents the least significant bit of the channel word, which is related to serial, synthetic data from the Data emulator.

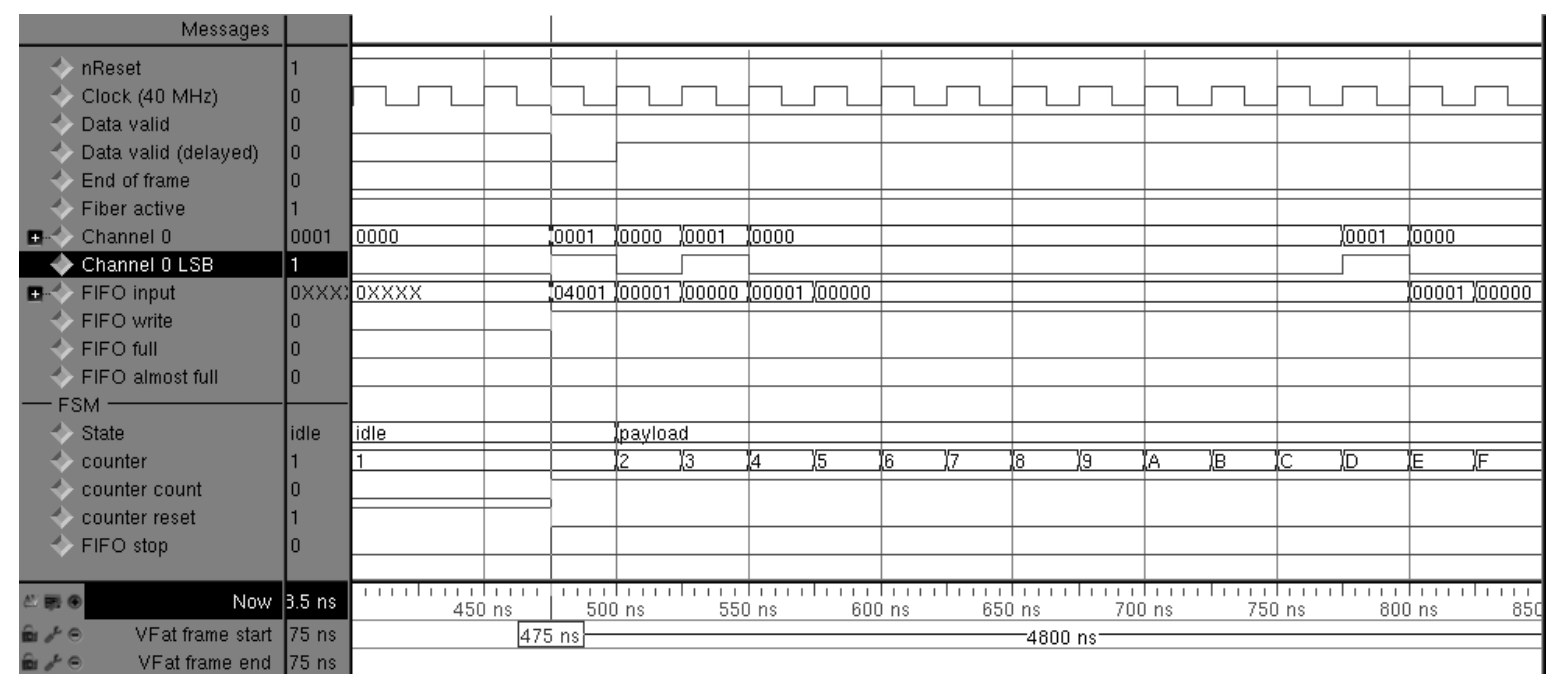

(a) Beginning

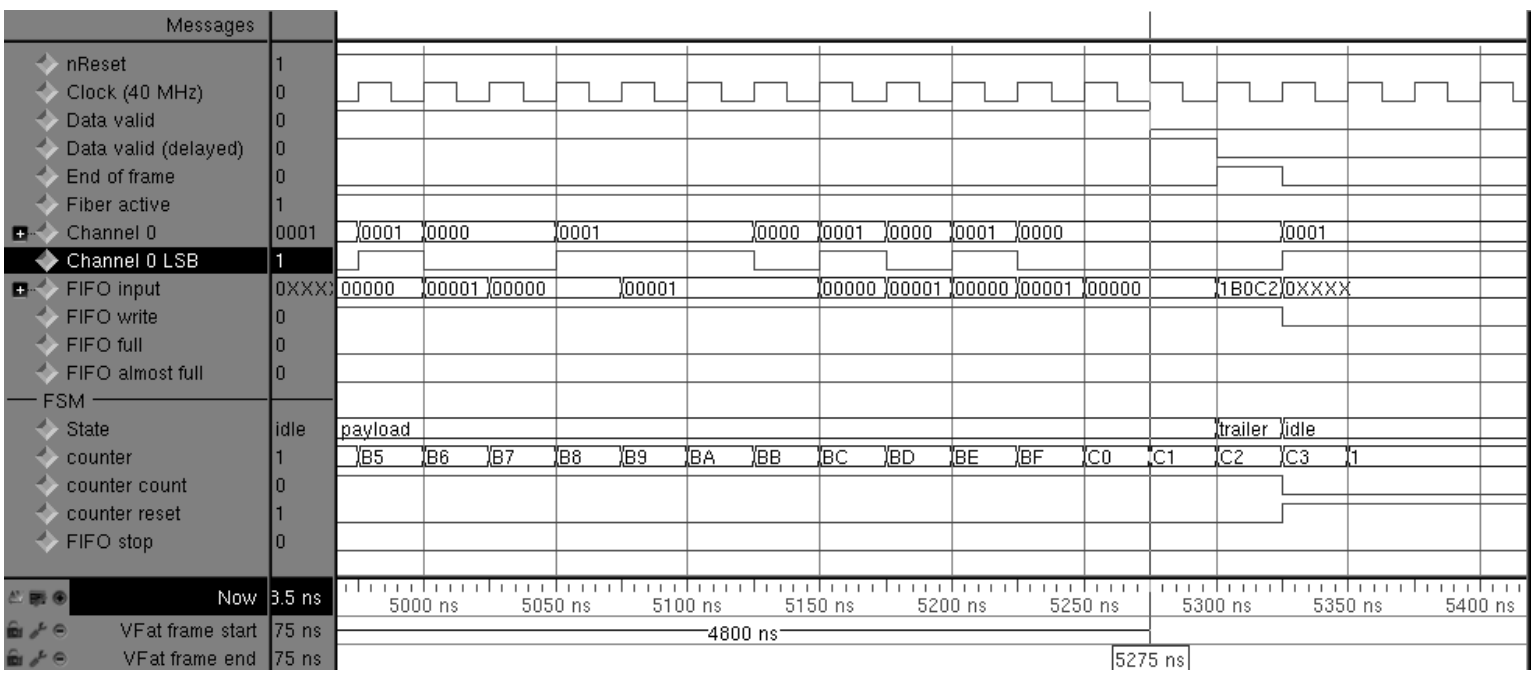

(b) End

Figure 6.20. Waveform presenting work of the synchronization cell.

\section{S-Link packet preparation block}

The second block in the processing entity is in charge of preparation of the S-Link packet. Figure 6.21 presents the diagram of the component. The input 
multiplexer chooses a data source for an embedded S-Link FIFO, which is capable to to store $4 \mathrm{k}$ of 65 bits words. The MSB of the data word from the FIFO is reserved for the UCTL(User control word) signal, according the S-Link 64 specification [11]. The flag is asserted by an Event Builder FSM accompanying a first and a last word of a frame. The rest of the bits is used as data.

The major multiplexer selects between a header, a trailer and three subframes sources related to the synchronization blocks. A part of a last word in the event frame is a TTS status (Section 2.3.4). It is specified by a TTS decoder. If values of the internal Event counter and the Event counter from the TTCrx Receiver differ, the decoder indicates the out of synchronization TTS state. Otherwise, if the busy line from the Main FPGA is asserted, the decoder pass this information to the trailer 20 . Eventually, if any of previous condition is satisfied, the decoder remain in ready state. The header and the trailer use information from other components ${ }^{21}$ to fill appropriate data filed (Table 6.2).

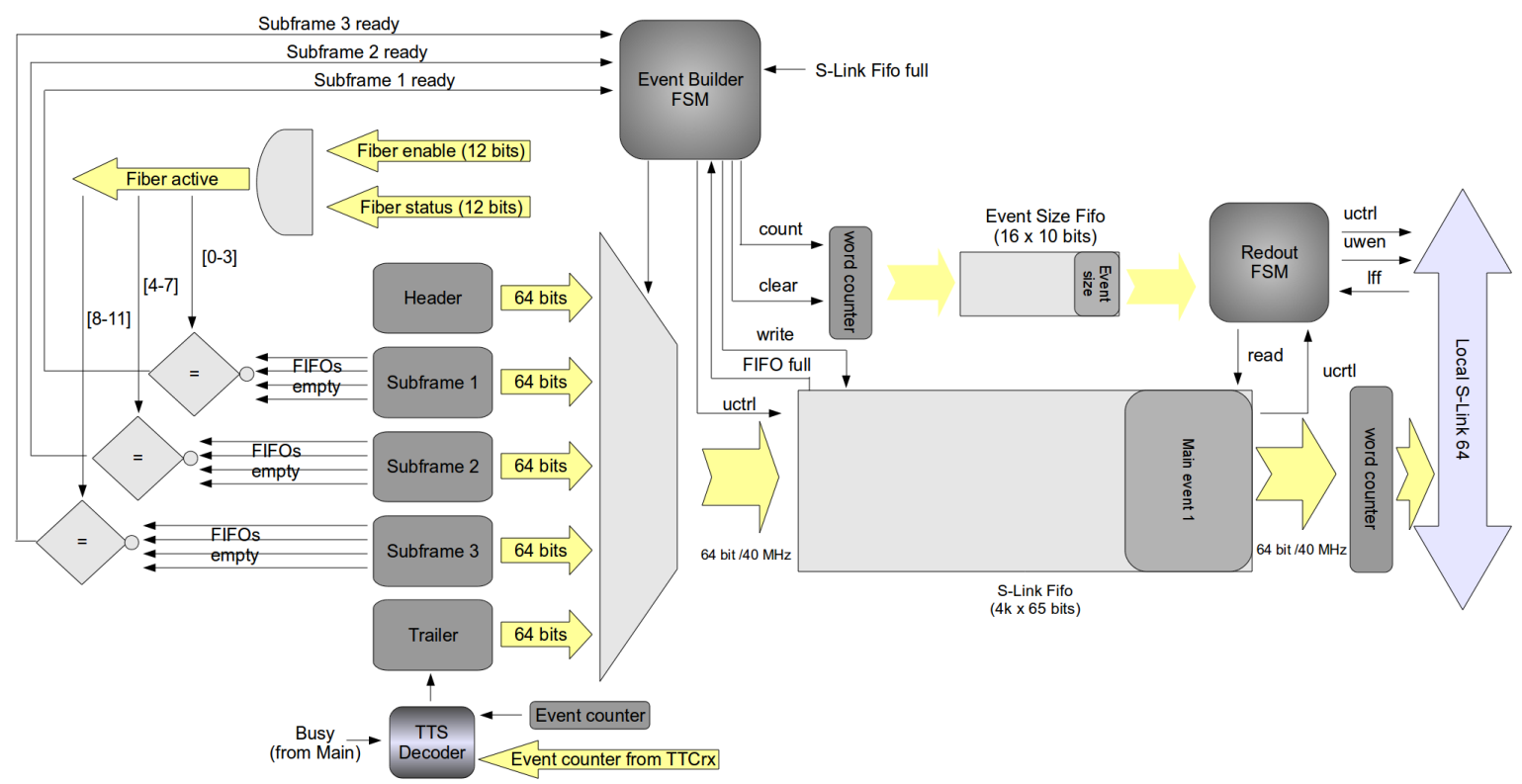

Figure 6.21. The S-Link packet preparation block from the processing entity.

Figure 6.4 demonstrates possibility of the skew between data from different GOH frames. For this reason, the Event Builder FSM needs the feedback information, if any subframe is already received. To obtain it, a negation of the empty flag from each basic cell of the synchronization block within a one subframe is compared with the respective Fiber active bit. As transmission of the GOH frame is continuous and both the synchronization and the S-Link preparation block work with the same clock, as soon as all synchronization FIFOs, that corresponds to the enabled channel within one subframe get first word from the GX receiver, the assigned Subframe ready flag is asserted and the FSM starts to copy the data to the S-Link FIFO.

Habitually, the word counter is used to count a number of word in a current event. Its values are stored in a Event Size Fifo capable to buffer 16 results (10

\footnotetext{
20 TTS state busy

21 like the TTCrx Receiver
} 
bits each). They are used by the Readout FSM, which is a master of the local implementation of the S-Link 64 interface.

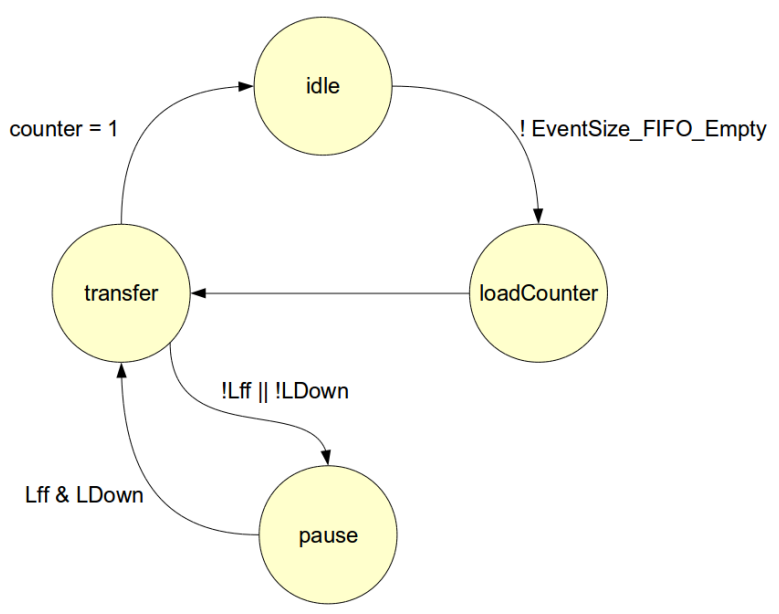

Figure 6.22. The state transition for the readout FSM from the S-Link preparation block.

Figure 6.22 presents the state transitions of the readout state machine. It uses a multiple FSM approach. For signals, which enables a communication via external interface, it exploits Moore's look-ahead style with registered outputs |22|. The signals driving internal components are generated with Mealy's approach.

The default state is the idle. The FSM checks if the Event Size Fifo is not empty. If there are any data in the FIFO, the read strobe of an internal counter is asserted and the FSM goes to loadCounter. In loadCounter the value from the Event Size Fifo is loaded to the counter and on a next rising edge of the clock, the state machine moves to the transfer. Back to the idle state is made when mentioned counter countdowns to 1 .

To accomplish correct reaction at the LFF (Link Full Flag and the LDOWN(Link Down), the FSM can move from the transfer to the pause state if either of the signals is low. As soon as their back high, the state machine resumes the data transfer. It allows to satisfy the requirement of the specification [11], to suspend a transfer at most after two clock cycles ${ }^{22}$ since the $L F F$ has been asserted.

The S-Link signals are routed through registers what contributes to achieving a correct timing 23 . The FSM uses directly the MSB of the S-Link Fifo to generate UCTL (User Control Flag), so additional logic, to distinguish first and last word, is not needed.

The state machine preparing the S-Link packet and storing it the FIFO is quite more complicated (Figure 6.23). It uses Mealy's approach. At the beginning, in the idle state, it waits for any subframe. However, subframes have priorities. The most privileged is the number one. If only the Subframe 1 ready flag is asserted and the S-link FIFO is not full, the FSM drives the read signal of the first four synchronization queues, writes header to the S-Link FIFO, starts counting a number of words and goes to the subframel state. If condition is not satisfied, the FSM checks the Subframe 2 ready flag. However, to make any other actions it needs

\footnotetext{
22 this implementation needs one

23 the fitter has a possibility to use flip-flops from IO blocks of a FPGA
} 


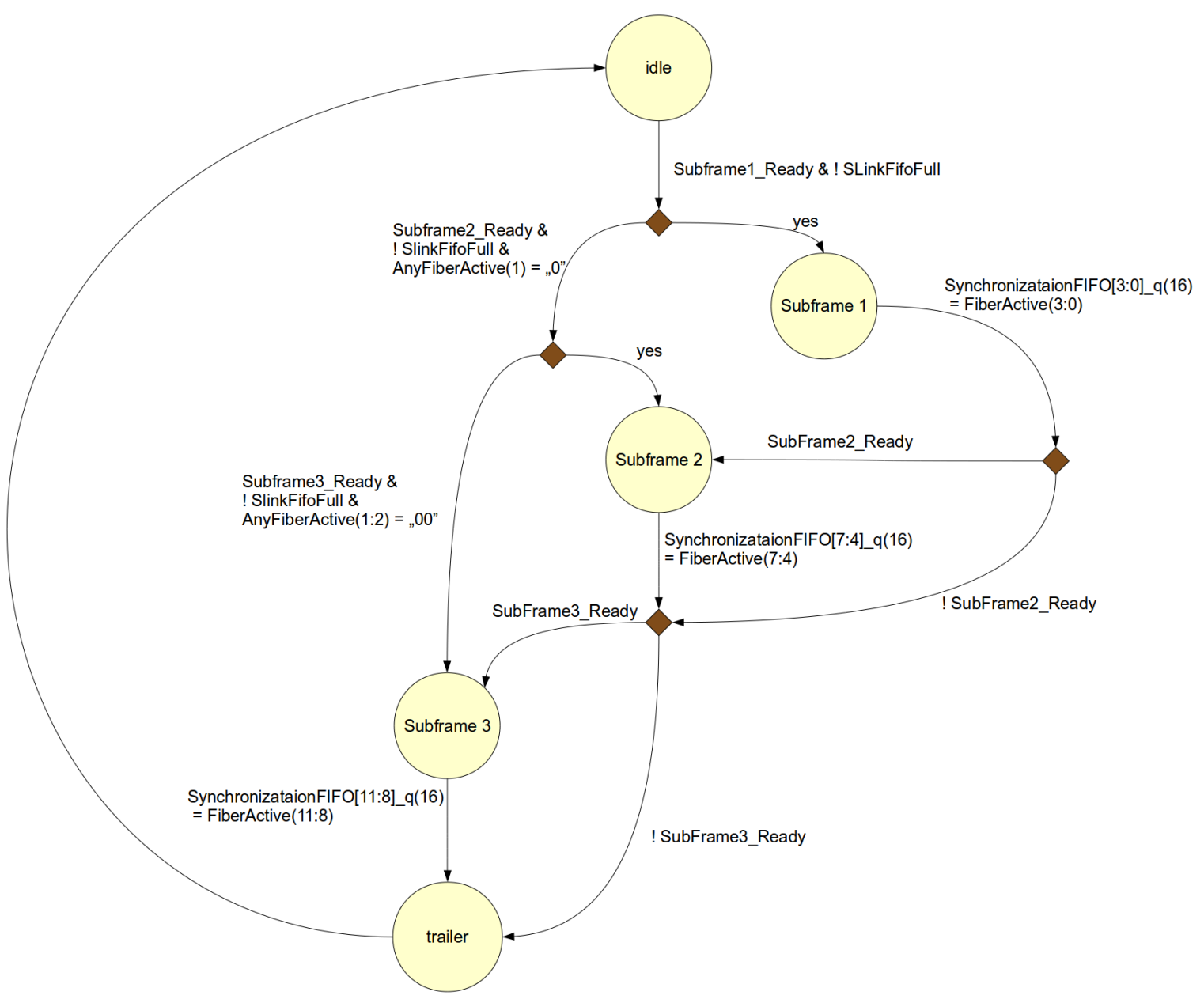

Figure 6.23. The state transition of the S-Link packet building state machine.

the anyFiberActive $1{ }^{24}$ signal. This is to protect the system against scattering data from the same physical collision in several frame events. It means, that even for asserted the Subframe 2 ready flag, if any of the first four fibers has been enabled by the user and a FPGA has managed to get a synchronization for this specific channels, the state machine will wait for data from them. The respective situation occurs for the subframe3 condition (Figure 6.23), anyhow, the FSM takes into account the anyFiberActive signal related to first eight fibers.

The list of actions performed by the FSM in the subrame1, the subrame2 and the subrame3 states is similar. It writes subsequent data from the appropriate synchronization FIFO into the S-Link FIFO, counting words and waiting until the $\mathrm{MSB}^{25}$ of the source queues fits to the pattern of the fiber active vector related to the subframe. Rooting the MSBs to the read port of its synchronization FIFOs resulting a flexible solution allowing to store $\mathrm{GOH}$ frames with different size ${ }^{26}$ within the same subframe. As soon as the FSM completes data copying of a one subframe it checks the subFrameReady flags for the rest maintaining the priority.

\footnotetext{
${ }^{24}$ Logical 'or' function of the first four denied fiber active

25 which indicates the end of frame

26 for example because of an error
} 
If any other is ready it asserts the appropriate read flags and goes to the specific state. If not, the state machine moves to the trailer state. Here, a trailer is written to the S-Link FIFO with the event and the bunch counter value. A number of words is stored in the event size queue. The FSM asserts the read acknowledge strobe for the TTCrxReceiver(Section 6.2.3) and goes back to the idle state.

\section{Example usage}

Figure 6.24 shows beginning of the event building. First, the FSM waits for any subframe ready. In $750 \mathrm{~ns}$ all of them are prepared by the synchronization cells. However, as the state machine has the priority list, it asserts the synchronization FIFO read strobe only for the four first queues. In the same time the OptoRx header in accompany of the active UCTRL flag is written to the SLink Data FIFO. On next rising edge of the UCLK the FSM moves to the subframe1 and starts copy following words. On the waveform is possible to observe an data encapsulation characteristic for the CMS Common Data Format - OptoRx header is followed by the GOH header, which is followed by the VFat one.

Figure 6.25 presents the situation, where the FSM is finishing the move of data to the SLink FIFO. After a last word from a third subframe, the state machine goes to the trailer state. The OptoRx trailer with the TTS status and length of the event is stored in queue. In the mean time, the TTS decoder asserts the TTCrx Receiver req strobe to obtain a following event and bunch counter value. What is more, the event length is written to the FIFO. The copy process of the event takes $14600 \mathrm{~ns}$.

As the Event Size FIFO is no longer empty, the readout FSM begins to work, what is showed on the waveform in Figure 6.26(a). It loads an internal counter with the number of words to send (15 $350 \mathrm{ns)} \mathrm{and} \mathrm{after} 2$ clock cycle of delay, starts transfer a S-Link frame. The header (15 $400 \mathrm{~ns}$ ) and trailer (30 $000 \mathrm{~ns}$ ) are correctly indicated by the low UCTRL. The S-Link access lasts again $14600 \mathrm{~ns}$.

The last waveform (Figure 6.27) presents behaves of the readout FSM at the LFF condition (15 $554 \mathrm{ns).} \mathrm{After} \mathrm{a} \mathrm{one} \mathrm{clock} \mathrm{cycle,} \mathrm{the} \mathrm{state} \mathrm{machine} \mathrm{suspend} \mathrm{the}$ transmission. As soon as $L F F$ is denied (15 $604 \mathrm{~ns}$ ), with delay of $25 \mathrm{~ns}$, the S-Llink communication is resumed. Although the pause, the frame is still consistent and has a correct width 27 .

$27154 \mathrm{~ns}+14396 \mathrm{~ns}=14600 \mathrm{~ns}$ corresponds to the value from the Figure 6.26 


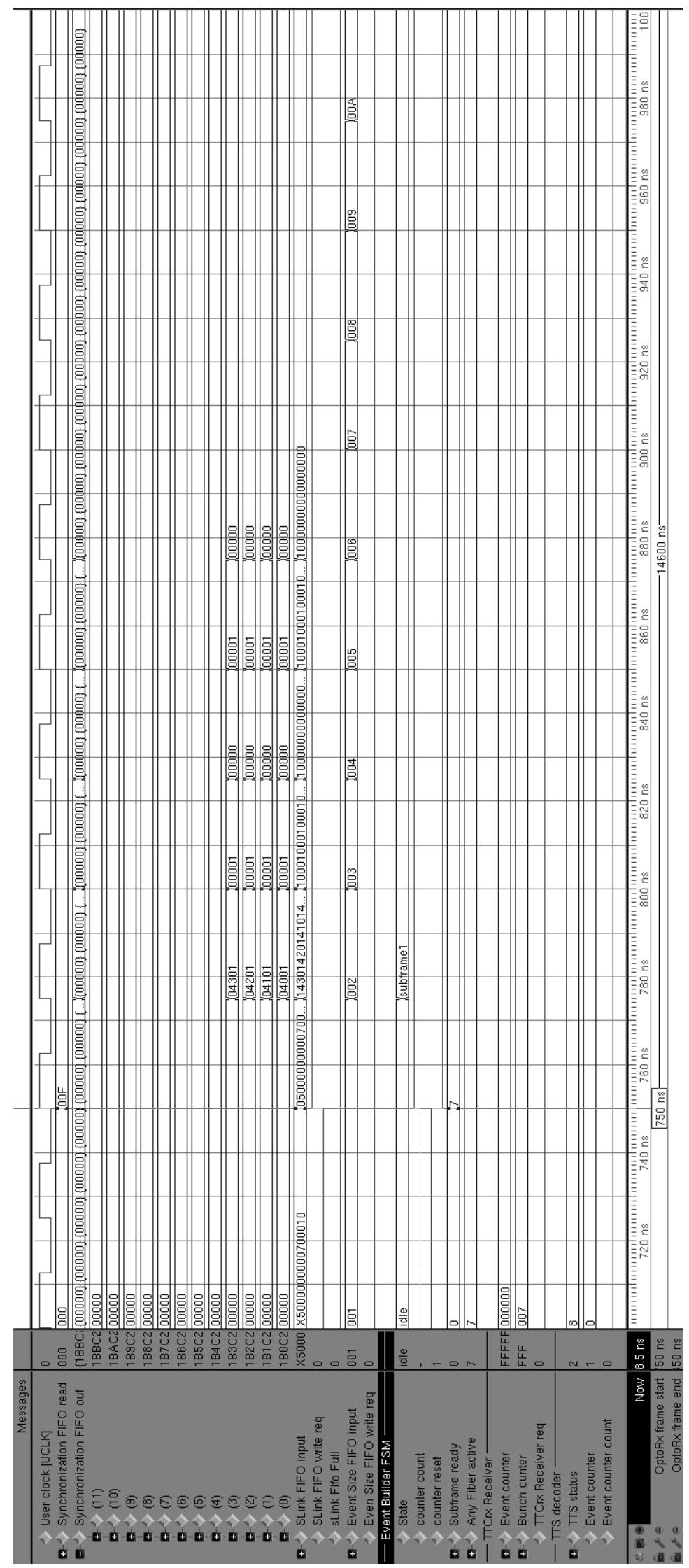

Figure 6.24. Waveform presenting work of the event builder. (Beginning) 


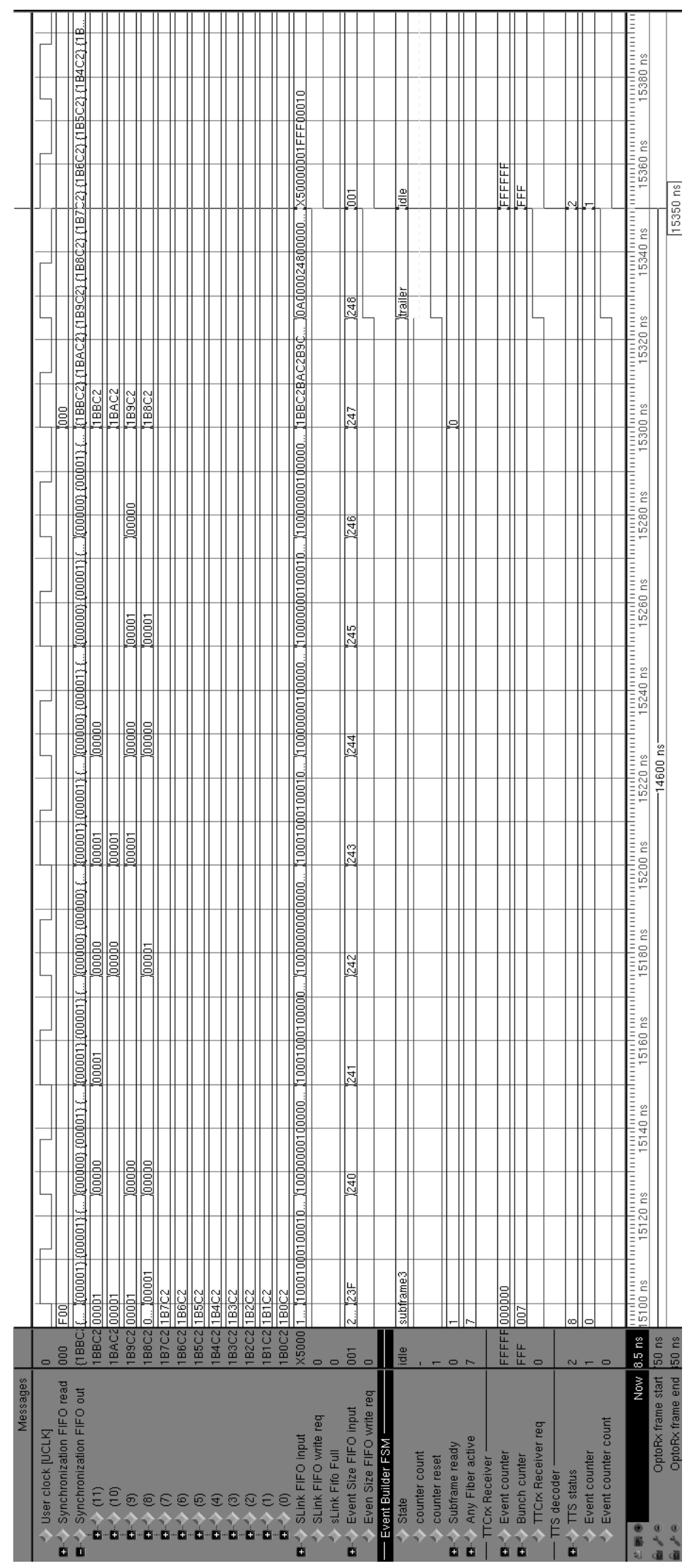

Figure 6.25. Waveform presenting work of the event builder. (End) 


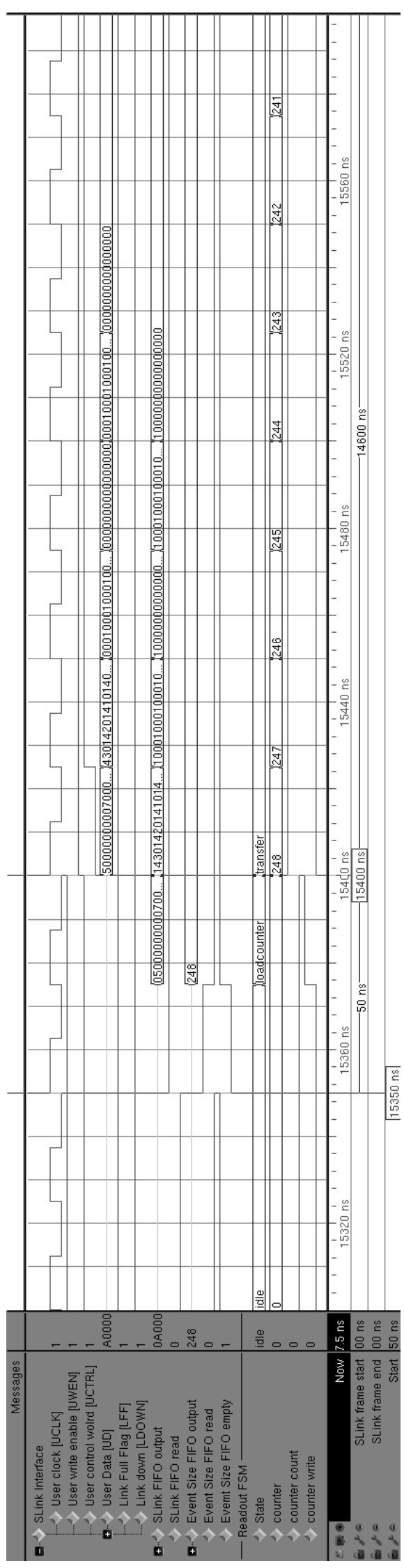

(a) Beginning

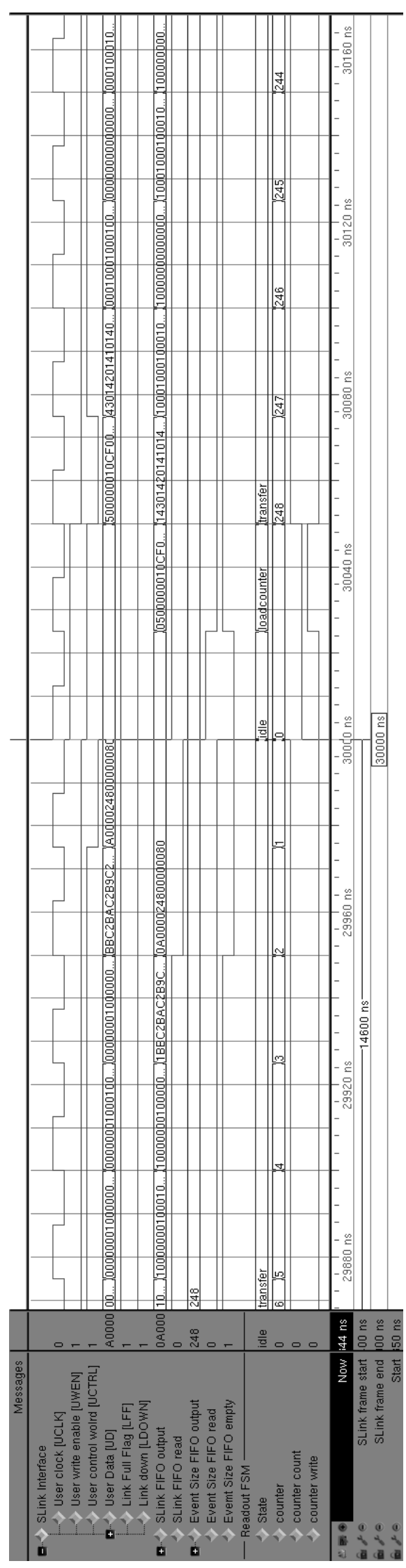

(b) End

Figure 6.26. Waveform presenting work of the readout FSM, which master the local S-Link interface. 


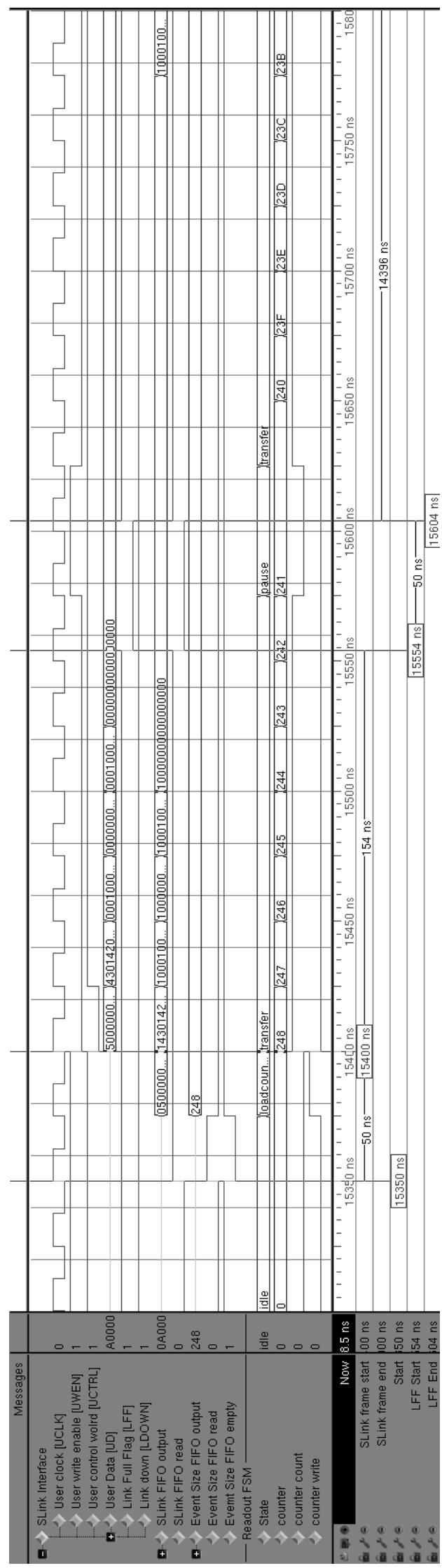

Figure 6.27. The waveform presenting reaction of the readout FSM at LFF (Link Full Flag) condition. 


\section{Data processing}

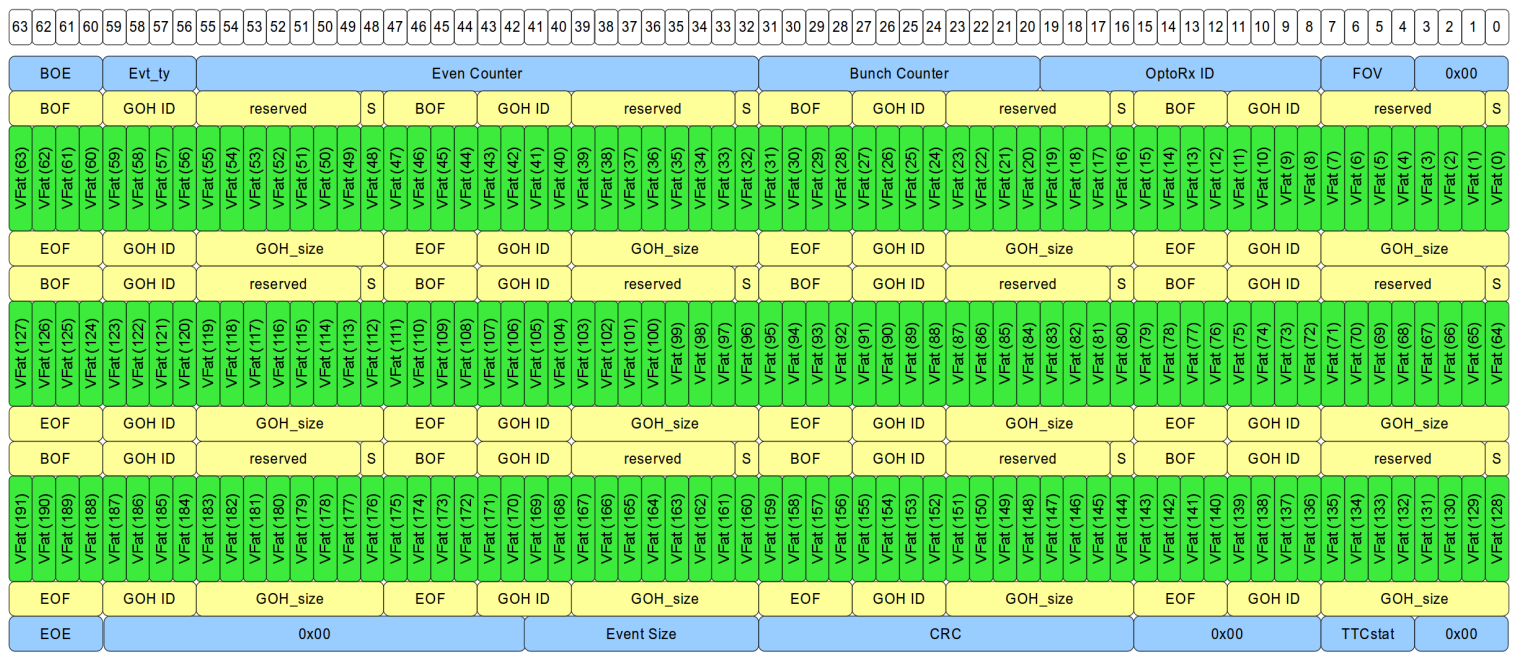

Figure 6.28. The output data format from OptoRx.

Figure 6.28 presents the complete event frame consistent with the CMS Common Data Format (Section 2.4 which one gets on the output of the S-Link Fifo. The header and the trailer ${ }^{28}$, which are added by the S-Link preparation block in the OptoRx, contain information needed by the software to manage data. The described data fields correspond to the packets scheme from Figure 2.8, Since the OptoRx handle the readout of 192 VFATs ${ }^{29}$ a simple solution is to format data in three data subframes, each containing 64 VFATs. Every subframe is tagged by a header and a trailer of the GOH frame added by the synchronization blocks ${ }^{30}$ to identify a source of data. The VFAT payload ${ }^{31}$ consist of 192 serial bits which structure was presented in the Figure 2.11. Table 6.2 describes data fields used in Figure 6.28

As each VME crate has assigned an independent computer which collects data and masters the VME bus, to reducem software development effort, the the TOTFed is polled by the PC to get data. To obtain the continuous and permanent readout, the data path on the TOTFed has several levels of buffering composed of FIFOs using an embedded memory in the FPGAs. It allows to work with the burst trigger, where average frequency of incoming events is $1 \mathrm{kHz}$ with a Poisson distribution, so few of frames can be very close to each other. Table 6.3 presents the following queues depth on the processing path.

The number of the events buffered at once by the TOTFed is limited by depth of the used event size FIFOs as well. Considering this, for the S-Link Packet buffer from the OptoRx, the maximum number of events is 16 and, whilst the Data Fifo from the Main limits this value to 256. However, achieve this level means the a problem of too short data frames and some error in the Data Acquisition.

\footnotetext{
28 marked in blue in Figure 6.28

2916 VFATs per GOH

30 marked in yellow

31 marked in green

32 Section 2.3.4

${ }^{33}$ All fibers are enabled
} 


\begin{tabular}{|c|c|c|c|}
\hline Identifier description & Field name & $\begin{array}{l}\text { length } \\
\text { [bits] }\end{array}$ & $\begin{array}{l}\text { Value } \\
\text { [hex] }\end{array}$ \\
\hline Beginning of Event Frame & $\mathrm{BOE}$ & 4 & $0 \times 5$ \\
\hline Event type & Evt_ty & 4 & $0 \mathrm{x} 0$ \\
\hline Number of L1A generated by TTC & Event counter & 24 & from the TTCrx receiver \\
\hline Bunch crossing counter & Bunch counter & 12 & from the TTCrx receiver \\
\hline OptoRx ID & OptoRx ID & 12 & programmed by software \\
\hline Version of data encapsulation & FOV & 4 & $0 \times 1$ \\
\hline Beginning of Frame & $\mathrm{BOF}$ & 4 & $0 \times 4$ \\
\hline GOH ID & GOH ID & 4 & from $0 \mathrm{x} 0$ to $0 \mathrm{xB}$ \\
\hline Fiber status & $\mathrm{S}$ & 1 & 0 -disabled 1 -active \\
\hline End of frame & EOF & 4 & $0 x B$ \\
\hline GOH frame length & GOH_size & 8 & 0xC2 (correct frame) \\
\hline End of Event & $\mathrm{EOE}$ & 4 & OxA \\
\hline $\begin{array}{l}\text { Length of the Event } \\
\text { (expressed in 64-bit words) }\end{array}$ & Event size & 10 & calculated \\
\hline CRC checksum & $\mathrm{CRC}$ & 16 & not implemented \\
\hline TTS status & TTCstat & 4 & from TTS decoder ${ }^{32}$ \\
\hline
\end{tabular}

Table 6.2. Description of the data fields from Figure 6.28 for the TOTFed

\begin{tabular}{l|c|c|c} 
Fifo name & FPGA & Size & Capacity in events ${ }^{33}$ \\
\hline Synchronization Fifo & OptoRx & 2k x 17 bits & 10 \\
S-Link Packet Fifo & OptoRx & $4 \mathrm{k}$ x 65 bits & 7 \\
Data Fifo & Main & $16 \mathrm{k}$ x 64 bits & 28
\end{tabular}

Table 6.3. Statistics of buffering FIFOs of the data path.

\section{Status LEDs}

The OptoRx board is equipped with 12 LEDs placed on the top side. The LED array shows the synchronization status of the fibers. Each LED is shining green if the corresponding fiber is in synch mode. In addition, also the TOTFed motherboard has a couple multi-colours LED in the front panel. Two of them are labelled as status $A$ and status $B$. The status $A$ LED indicates the behaviour of the fiberActive signal and the status $B$ one reports the mode of the OptoRx. The detailed description is presented in Table 6.4.

\begin{tabular}{c|c|l} 
Led label & Colour & Description \\
\hline Status A & $\begin{array}{c}\text { Red } \\
\text { Orange } \\
\text { Green }\end{array}$ & $\begin{array}{l}\text { The enabled fibers are not in synch or no fiber is selected. } \\
\text { Only some of the enabled fibers are in synch. } \\
\text { All enabled fibers are in synch. }\end{array}$ \\
\hline Status B & Red & $\begin{array}{l}\text { The readout queue (Main FPGA and OptoRx) reaches a BUSY } \\
\text { condition which stopped the trigger through the TTS. } \\
\text { OptoRx in data emulation mode. } \\
\text { Orange } \\
\text { OptoRx in real data taking mode. }\end{array}$
\end{tabular}

Table 6.4. Description of the LEDs in the front panel of the TOTFed. 


\section{Summary}

\subsection{Synthesis}

The project was synthesized in the Quartus II 9.0 sp2 64 bit environment. Tables 7.1 7.3 present the obtained results.

\begin{tabular}{l|l|l|l|l} 
Component name & Instance name & $\begin{array}{l}\text { Number } \\
\text { of LCS }\end{array}$ & $\begin{array}{l}\text { Number of LC } \\
\text { registers }\end{array}$ & Memory \\
\hline fpgaReload & fpgaReload_inst & 6 & 13 & 0 \\
jTagController & jTagController_inst & 35 & 32 & 0 \\
OneWireMaster & OneWireMaster_inst & 1484 & 887 & 0 \\
TTCrx_I2C_Controller & TTCrx_I2C_Controller_inst & 127 & 76 & 0 \\
VME_CONT & VMEController_inst & 822 & 225 & 0 \\
vmepll & vmepll_inst & 0 & 0 & 0 \\
VMEResetSubsystem & VMEResetSusbsystem_inst & 14 & 10 & 0 \\
VME (all) & & 2527 & 1270 & 0 \\
& & $(63 \%)$ & &
\end{tabular}

Table 7.1. Resources usage in the VME FPGA.

Table 7.1 shows that the biggest component in the VME FPGA is the One Wire Master. It was not described in this document, because the author have not participated in its implementation. The intense resource utilization for such a simple interface is caused by the coding style - the VHDL code of this module has been written at a high abstraction level. The refactoring of the One Wire Master is planned for the further stage of the firmware project, since, despite not being optimal, the module works correctly from the functional point of view and moreover it is not crucial for DAQ system.

Several components were not described in this document, because of their simplicity. The fpgaReload from Table 7.1 is supposed to enable a remote reload request from the VME bus. However, it is not yet implemented and the current version protects the system against an accidental access. The jTagController bridges the internal bus of the VME with a jTag TAP Master (LVT8990 [23]) produced by Texas. As it is a simple wrapper only linking two interfaces, it does not require a lot of resources. It will be useful in the imminent releases of firmware and software, allowing remote programming of the TOTFed located in the experiment zone from the control room.

$64 \%$ of the Main FPGA usage falls in reasonable limits - it still gives a chance to the fitter to scatter modules within a die of a FPGA and, as utilization of rooting resources in not to high, to obtain good timing.

\footnotetext{
${ }^{1}$ Logic Cell
} 


\begin{tabular}{|c|c|c|c|c|}
\hline Component name & Instance name & $\begin{array}{l}\text { Number } \\
\text { of LCs }\end{array}$ & $\begin{array}{l}\text { Number } \\
\text { of LC } \\
\text { registers }\end{array}$ & $\begin{array}{l}\text { Memory } \\
\text { (bits) }\end{array}$ \\
\hline LocalBusSlave & Local_Bus_Interface & 86 & 38 & 0 \\
\hline MainResetSubSystem & MainResetSubSystem_inst & 16 & 10 & 0 \\
\hline OptoRxReceiver & OptoRX & 595 & 537 & 1052672 \\
\hline MainPll & PLL & 1 & 0 & 0 \\
\hline TTS & TTS_inst & 2 & 1 & 0 \\
\hline VHDL Main (all) & & $\begin{array}{l}771 \\
(4 \%)\end{array}$ & 601 & $\begin{array}{l}1052672 \\
(63 \%)\end{array}$ \\
\hline
\end{tabular}

Table 7.2. Resources usage in the Main FPGA.

Table 7.2 presents usage of the Main. It proves the major objective of the FPGA, which is mainly supposed to buffer the data. The utilization is at a very low level, so the fitter does not have a problem related to lack of rooting resources.

\begin{tabular}{l|l|l|l|l} 
Component name & Instance name & $\begin{array}{l}\text { Number } \\
\text { of } \\
\text { ALUTs }\end{array}$ & $\begin{array}{l}\text { Number } \\
\text { of reg- } \\
\text { isters }\end{array}$ & $\begin{array}{l}\text { Memory } \\
\text { (bits) }\end{array}$ \\
\hline ch_data_processor & InputCH:0:DP & 181 & 237 & 576 \\
ch_data_processor & InputCH:1:DP & 185 & 237 & 567 \\
ch_data_processor & InputCH:2:DP & 185 & 237 & 567 \\
ch_data_processor & InputCH:3:DP & 181 & 237 & 567 \\
ch_data_processor & InputCH:4:DP & 172 & 237 & 567 \\
ch_data_processor & InputCH:5:DP & 172 & 237 & 567 \\
ch_data_processor & InputCH:6:DP & 171 & 237 & 567 \\
ch_data_processor & InputCH:7:DP & 174 & 237 & 567 \\
ch_data_processor & InputCH:8:DP & 187 & 237 & 567 \\
ch_data_processor & InputCH:9:DP & 188 & 237 & 567 \\
ch_data_processor & InputCH:10:DP & 196 & 237 & 567 \\
ch_data_processor & InputCH:11:DP & 187 & 249 & 567 \\
LocalBusSlave & Opto_Local_Bus_Interface & 13 & 20 & 0 \\
led_driver & LD & 22 & 0 & 0 \\
OptoRxResetSubSystem & OptoRxResetSubSystem_inst & 17 & 11 & 0 \\
processing & proc & 1740 & 2007 & 684192 \\
Trigger_emu & TE & 5 & 7 & 0 \\
TTCrx_receiver_4bit & TTCrx_4bit & 108 & 172 & 4608 \\
pll & U1 & 0 & 0 & 0 \\
s2gxBasic & U5 & 0 & 0 & 0 \\
OptoRx (all) & & $(10 \%)$ & 6161 & 6957712 \\
& & $(13 \%)$ & $(27 \%)$
\end{tabular}

Table 7.3. Resources usage in the OptoRx FPGA.

Table 7.3 reports on the OptoRx FPGA. The ch_data_processors block links the $8 b / 16 b$ converter, the bridge FIFOs and the VFAT emulator. The s2gxBasic is related to the GX receivers.

Although the entire data processing from 12 channel occurs in this device, as the Stratix II GX devices are dedicated for complex digital signal application, the utilization of a chip is very low. It contributes also to excellent timing results. 
To be sure that, that there is no timing violation ${ }^{2}$, the author specified in an industry-standard Synopsys Design Constraints (SDC) [24] format (.sdc files) timing requirements for external signals. It guides the fitter, which is an input and output delay ${ }^{3}$ of specific ports and which of them are used as a clock reference. Thanks to that, the tool has an opportunity to place logic in such a way, that all requirements are met. Moreover, the TimeQuest Timing Analyzer [25] from Quartus packet provides a comprehensive report in which the time issues can by analysed and, if it is needed, the design can be rebuilt to finally satisfy the requirements.

\subsection{Real life tests}

The on-line part of the DAQ of the TOTEM experiment, at first stage of commissioning, should be able to work with the $1 \mathrm{kHz}$ trigger. The processing part of the data path is pipelined and needs at most 14.6 us ${ }^{4}$ to build a correct event and send it from one buffer to other. A whole event from the TOTFed consists of two frames of the OptoRx. As 14.6 us is negligible, the fulfilment of the trigger requirements depends only on a speed of the VME and the Local bus. The event 5 consists of $582 * 8=4656$ bytes. Two events take 9312 bytes. Each subdetector in the experiment uses two TOTFeds to collect data, which results in 18.624 bytes per single event. It means that theoretical speed limit is around $18 \mathrm{MB} / \mathrm{s}$.

To check the theoretical speed under laboratory conditions, the author performed a test by reading in the VME block transfer mode ${ }^{6}$ value for the DEBUG COUNTER of each FPGA. The obtained results are presented in Table 7.4. Furthermore, as counter changes its value with each access, it was a simple way to check the data consistency.

\begin{tabular}{l||l} 
Device & Average Speed [MB/s] \\
\hline Main 1 & 23.71 \\
Main 2 & 23.64 \\
Main 3 & 23.63 \\
\hline OptoRx 1 & 20.50 \\
OptoRx 2 & 20.70 \\
OptoRx 3 & 19.42
\end{tabular}

Table 7.4. Average speed of the DEBUG COUNTER readout.

To evaluate if the speed requirement is satisfied, one should take in account the results related to the Mains 7 . The test confirmed that designed solution meets the bandwidth limit.

Figure 7.1 presents the average speed of the Roman Pot motherboard [26] readout. The test was performed under laboratory conditions using the VBT-325C VME Bus Analyzer [27] to measure the performance. The obtained result proves the

\footnotetext{
${ }^{2}$ specially related to the Local bus which is critical for the data acquisition on the TOTFed

3 associated to the estimated delay of the electric lines on the board, internal delay of an external buffers (ABT16245) and the different FPGAs

4 according to the waveform 6.26

5 according to Figure 6.28

61000 bytes per one block

7 the software to collect data reads FIFOs of this devices
} 


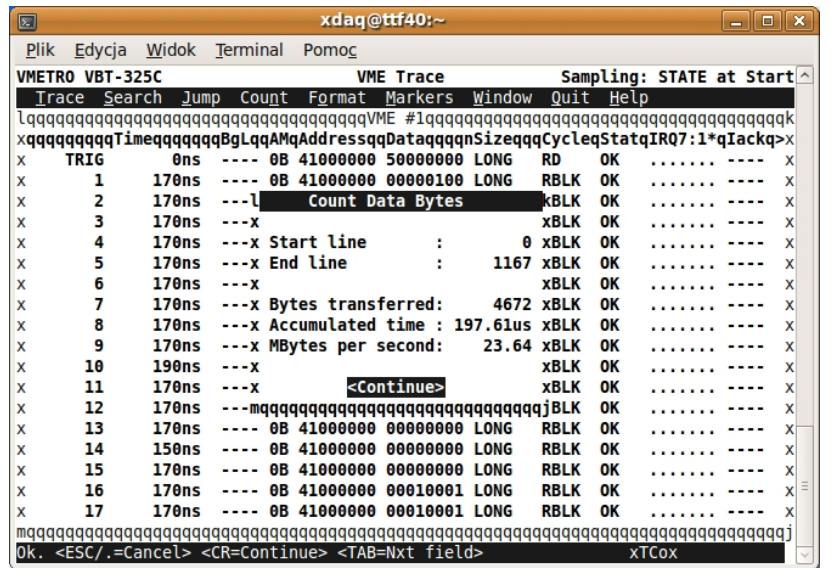

Figure 7.1. Average speed of the Roman Pot motherboard readout.

values from the previous test. In the background, the OptoRx frame beginning together with the header can be seen.

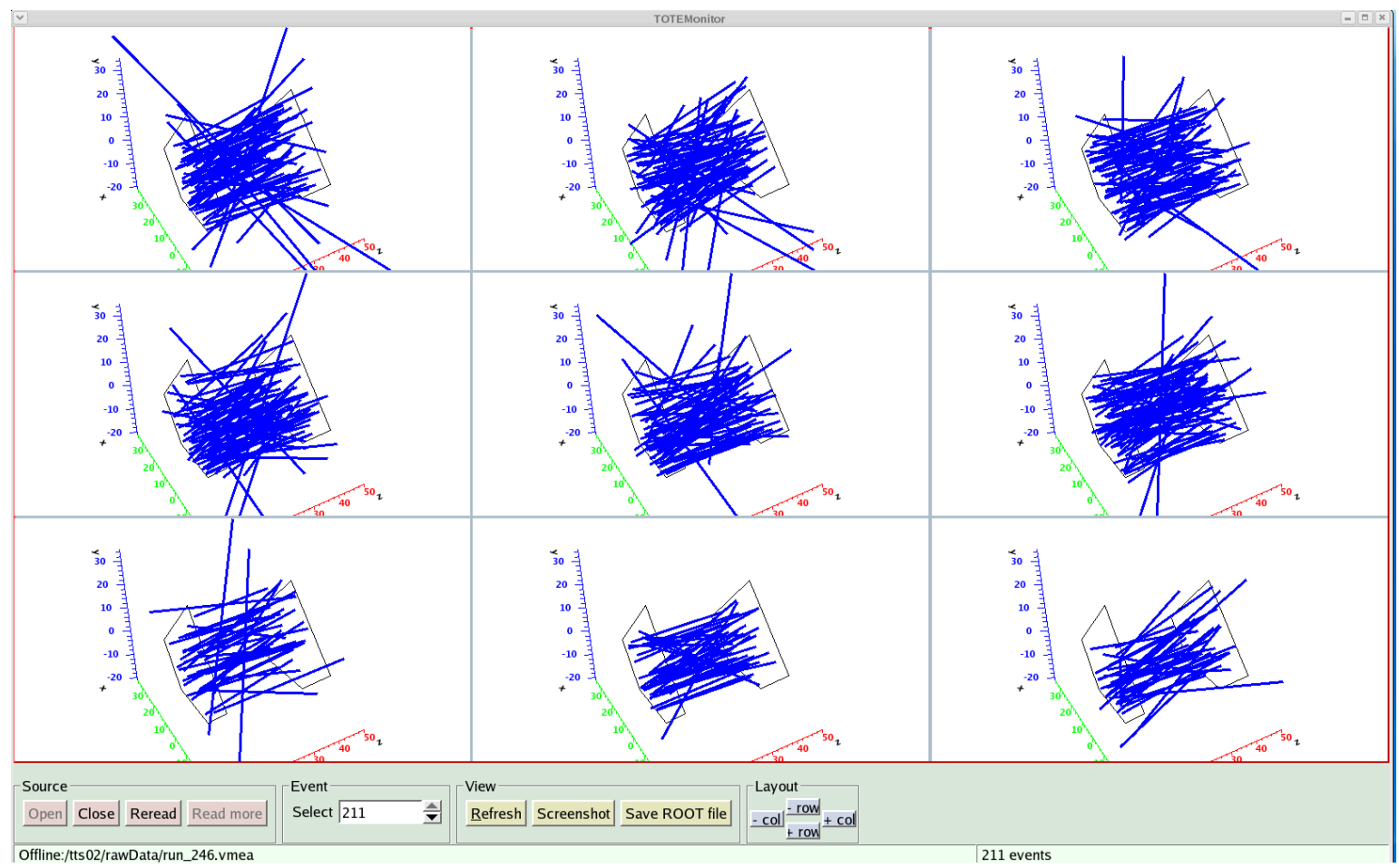

Figure 7.2. One of the first tracks observed in the TOTEM experiment - detector Roman Pot, TOTEM On-line Monitoring.

The TOTEM has started the regular runs on March 2010 with the center of the mass energy of $7 \mathrm{TeV}$ per collision. For the moment, it is hard to evaluate the performance of the front-end driver in the LHC experiment because of a very low trigger frequency (at most $100 \mathrm{~Hz}$ )

\footnotetext{
8 The TOTEM experiment is still in the commissioning phase and the T1 telescope is not yet installed in the LHC tunnel.
} 
one of the first tracks observed in the Roman Pot detector. Each trapezium is related to the silicon detector of the Roman Pot placed in the vicinity of the beam. Blue lines represent the tracks of particles after the collision.

\subsection{Conclusions}

While preparing the thesis, the author had an opportunity to work with real hardware in the biggest physics laboratory of the world. The cooperation within an international team was a exhilarating and valuable experience. The complexity and stringent requirements of the project allowed the author to gain valuable experience in the field of FPGA programming.

The author learned how to:

- write a synthesize-able, transparent code and predict $r t l$ results;

- choose a proper FSM approach according to the application;

- cope with an external communication between FPGAs;

- perform a static timing analysis;

- prepare testbenches on a rtl and a gate levels;

- use basic $t c l$ to automatize work with EDA tools;

- use internal resources of the FPGA (for example GX transceivers, an embedded memory);

— organize a big design;

- use tools facilitating work of a team of people under one project (for example sun);

The thesis gave a unique chance to be involved in the High Energy Physics experiment at the biggest accelerator of the world. The author have got acquainted with VME specification and other interfaces used in such a field of electronics.

If the author had an opportunity to start his work now from the scratch, he would begin of designing the TOTFed motherboard in a different way, exploiting the stole of the art FPGA technology. He would use a single FPGA, for example EP4SGX230KF40 from the Stratix IV GX family. It has enough number of transceivers (36) and logic resources. Nowadays, the price of such an FPGA is equivalent to the costs of all actually used programmable devices. The Stratix IV GX equipped with $8 \mathrm{MB}$ of SRAM would allow for a much more flexible solution. First of all, the single chip approach eliminates the problem of external delays, which significantly decrease the performance. It reduces the design effort, because data are not transmitted between different devices on the board, but buffered together. A designer need not to worry about consecutive output interfaces, but can focus on efficient implementation of the data processing. Furthermore, at each level of the project, the internal data flow and architecture can be changed. The debugging of such a solution is simplified because the producers provide the some kind of a chip scope. Finally, the static timing analysis is much simpler and more clear for the case of a single FPGA.

Although the design meets for a moment all the requirements and allows to collect consistent data it is not finished yet. As the experiment is being constantly commissioned, also the firmware needs continuous updates. In the future, TOTEM will work with a trigger of $100 \mathrm{kHz}$, when the physics data will be reordered together with the CMS experiment. It means a change of the data flow. The VME bus will be used only to configure the TOTFed. The external S-Link interface will be connected 
to the CMS system so full compatibility with the CMS project will be mandatory. Moreover, according to the requirements, the event size from the OptoRx will have to be reduced to $2 \mathrm{kB}$ so the processing part will need to compress the amount of data 9 . Finally, the TOTFed will check data consistency on the fly.

The author hopes that effort put in the described project provides a good starting point for future changes and, in the end, the TOTEM experiment will allow the humanity to understand better the surrounding world and contribute to the development of knowledge and technology.

9 right now there is a redundancy in the OptoRx frame, for example each VFat frame provides the bunch and event number, which are the same within one event for all of them 


\section{Bibliography}

[1] TOTEM Collaboration. The TOTEM Experiment at the CERN Large Hadron Collider. Jinst, 2008. http://www.iop.org/EJ/abstract/1748-0221/3/08/S08007.

[2] TOTEM Collaboration. TOTEM Technical Design Report. Technical report, 2004. http://totemtdr.web.cern.ch/totemtdr/totemtdr24-03-04.pdf.

[3] Hubert Niewiadomski. Reconstruction of Protons in the TOTEM Roman Pot Detectors at the LHC. PhD thesis, University of Manchester, 2008. Chapter 3.

[4] Leila Belkora (ed.) Doreen Wackeroth. Cross section. High Energy Physics Made Painless, 1996.

[5] E. Oliveri. The Forward Inelastic Telescope T2 for the TOTEM Experiment at the LHC. $\mathrm{PhD}$ thesis, University of Siena, 2010.

[6] Cheuk-Yin Wong. Introduction to High-Energy Heavy-Ion Collisions, page 24. Word Scientific Publishing Co. Pte. Ltd., 1994.

[7] P. Moreira, T. Toifl, A. Kluge, G. Cervelli, A. Marchioro, and J. Christiansen. GOL Reference Manual. CERN, 2005.

http://proj-gol.web.cern.ch/proj-gol/manuals/gol_manual.pdf.

[8] Technical Committee on Microprocessors and Microcomputers of the IEEE Computer Society. IEEE Standard for a Versatile Backplane Bus: VMEbus (ANSI/IEEE Std 1014-1987). Technical report, R2008.

[9] CMS Collaboration. Trigger, Timing and Control interface.

http://ttc.web.cern.ch/TTC/.

[10] CMS Collaboration. The Front-End Control (FEC).

http://proj-fec-ccs.web.cern.ch/proj-FEC-CCS/.

[11] Robert McLaren \& Erik van der Bij Owen Boyle. S-Link 64 Specification. Technical report, 1997.

http://www94.web.cern.ch/HSI/s-link/spec/.

[12] CMS Collaboration. The VHDL implementation of the CRC.

http://cmsdoc.cern.ch/cms/TRIDAS/horizontal/RUWG/session19/crc_usb_ en.vhd,

[13] P. Aspell, G. Anelli, P. Chalmet, J. Kaplon, K. Kloukinas, H. Mugnier, W. Snoeys. VFAT2 : A Front-end system on chip providing fast trigger information, digitized data storage and formatting for the charge sensitive readout of multi-channel silicon and gas particle detectors. CERN.

http://cdsweb.cern.ch/record/1069906/files/p292.pdf.

[14] CAEN. Technical Information Manual of VME-PCI Optical Link Bridge (V2718), 2009. http://www.caen.it/getattach.php?mod=V2718\&obj=mn\&id=2250.

[15] Paulo Moreira. BPLL Manual. CERN. http://proj-qpll.web.cern.ch/proj-qpll/images/qpllManual.pdf.

[16] Wade D. Peterson. The VMEbus Handbook. VFEA International Trade Association, 1993.

[17] J. Christiansen, A. Marchioro, P. Moreira and T. Toifl. TTCrx Reference Manual. CERN, 2005.

www.cern.ch/TTC/TTCrx_manual3.11.pdf

[18] Altera. Cyclone FPGA Family Data Sheet, 2008.

http://www.altera.com/literature/hb/cyc/cyc_c5v1_01.pdf. 
[19] The I2C-Bus Specification and user manual. Technical report, 2007. WWW.nxp.com/documents/user_manual/UM10204.pdf.

[20] Altera. Stratix Device Handbook, 2005.

http://www.altera.com/literature/hb/stx/stratix_handbook.pdf.

[21] Altera. Stratix II GX Device Handbook, 2009. http://www.altera.com/literature/hb/stx2gx/stxiigx_handbook.pdf.

[22] Chu Pong P. RTL Hardware design using VHDL. A John Wiley \& Sons, INC., 2006.

[23] Texas Instruments. SN54LVT8980, Embedded Test-Bus Controllers, 1997. http://www.ti.com/lit/gpn/sn74lvt8980.

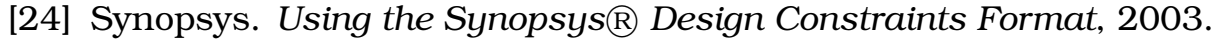
http://www.ing2.unirc.it/portale/didattica/files_docenti/ 6131520080418112629.pdf.

[25] Altera. The Quartus II TimeQuest Timing Analyzer, 2008. http://www.altera.com/literature/hb/qts/qts_qii53018.pdf.

[26] G. Antchev, T. Kiss, W. Snoeys. The TOTEM Roman Pot Motherboard. In Topical Workshop on Electronics for Particle Physics, Greece, Sep 2008. http://doc.cern.ch//archive/cernrep/2008/2008-008/p446.pdf.

[27] VMetro. Manual of the VBT-325C (VME Bus Analyser), 1997. http://www-cdfonline.fnal.gov/daq/commercial/VBT-325.pdf. 